\title{
Semi-inclusive deep-inelastic scattering in Wandzura-Wilczek-type approximation
}

\author{
S. Bastami, ${ }^{a}$ H. Avakian, ${ }^{b}$ A.V. Efremov, ${ }^{c}$ A. Kotzinian, ${ }^{d, e}$ B.U. Musch, ${ }^{f}$ \\ B. Parsamyan, ${ }^{l, e}$ A. Prokudin, ${ }^{g, b}$ M. Schlegel, ${ }^{h}$ G. Schnell, ${ }^{i, j}$ P. Schweitzer ${ }^{a, k}$ \\ and K. Tezgin ${ }^{a}$ \\ ${ }^{a}$ Department of Physics, University of Connecticut, Storrs, CT 06269, U.S.A. \\ ${ }^{b}$ Thomas Jefferson National Accelerator Facility, Newport News, VA 23606, U.S.A. \\ ${ }^{c}$ Joint Institute for Nuclear Research, Dubna, 141980 Russia \\ ${ }^{d}$ Yerevan Physics Institute, Alikhanyan Brothers St., 375036 Yerevan, Armenia \\ ${ }^{e}$ INFN, Sezione di Torino, 10125 Torino, Italy \\ ${ }^{f}$ Institut für Theoretische Physik, Universität Regensburg, 93040 Regensburg, Germany \\ ${ }^{g}$ Division of Science, Penn State Berks, Reading, PA 19610, U.S.A. \\ ${ }^{h}$ Department of Physics, New Mexico State University, Las Cruces, NM 88003-001, U.S.A. \\ ${ }^{i}$ Department of Theoretical Physics, University of the Basque Country UPV/EHU, \\ 48080 Bilbao, Spain \\ ${ }^{j}$ IKERBASQUE, Basque Foundation for Science, 48013 Bilbao, Spain \\ ${ }^{k}$ Institute for Theoretical Physics, Universität Tübingen, D-72076 Tübingen, Germany \\ ${ }^{l}$ CERN, 1211 Geneva 23, Switzerland \\ E-mail: saman.bastami@uconn.edu, avakian@jlab.org, \\ efremov@theor.jinr.ru, aram.kotzinian@cern.ch, bmusch@b-mu.de, \\ bakur@cern.ch, prokudin@jlab.org, schlegel@nmsu.edu, \\ gunar.schnell@desy.de, peter.schweitzer@phys.uconn.edu, \\ kemal.tezgin@uconn.edu
}

ABSTRACT: We present the complete cross-section for the production of unpolarized hadrons in semi-inclusive deep-inelastic scattering up to power-suppressed $\mathcal{O}\left(1 / Q^{2}\right)$ terms in the Wandzura-Wilczek-type approximation, which consists in systematically assuming that $\bar{q} g q$-terms are much smaller than $\bar{q} q$-correlators. We compute all twist-2 and twist-3 structure functions and the corresponding asymmetries, and discuss the applicability of the Wandzura-Wilczek-type approximations on the basis of available data. We make predictions that can be tested by data from COMPASS, HERMES, Jefferson Lab, and the future Electron-Ion Collider. The results of this paper can be readily used for phenomenology and for event generators, and will help to improve the description of semi-inclusive deep-inelastic processes in terms of transverse momentum dependent parton distribution functions and fragmentation functions beyond the leading twist.

KEYWords: Deep Inelastic Scattering (Phenomenology), QCD Phenomenology

ArXiv EPRINT: 1807.10606 


\section{Contents}

1 Introduction 1

2 The SIDIS process in terms of TMDs and FFs 3

2.1 The SIDIS process 3

$\begin{array}{lll}2.2 & \text { TMDs, FFs and structure functions } & 6\end{array}$

3 WW and WW-type approximations 11

$\begin{array}{lll}3.1 & \text { WW approximation for PDFs } & 12\end{array}$

$\begin{array}{lll}3.2 & \text { WW-type approximations for TMDs and FFs } & 12\end{array}$

$\begin{array}{lll}3.3 & \text { Predictions from instanton vacuum model } & 14\end{array}$

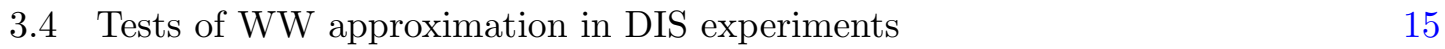

$\begin{array}{ll}3.5 & \text { Tests in lattice QCD } \\ & 16\end{array}$

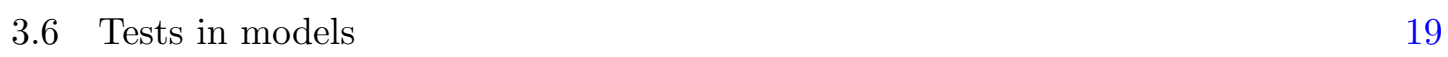

3.7 Basis functions for the WW-type approximations 20

3.8 Limitation of WW-type approximations 21

4 SIDIS in the WW-type approximation and Gaussian model 22

4.1 Leading structure functions amenable to WW-type approximations 22

4.2 Subleading structure functions in WW-type approximations 23

4.3 Gaussian Ansatz for TMDs and FFs 23

4.4 Evaluation of structure functions in WW-type \& Gaussian approximation 24

$\begin{array}{ll}\text { 4.5 Phenomenological information on basis functions } & 25\end{array}$

5 Leading-twist asymmetries and basis functions $\quad 26$

5.1 Leading-twist $F_{U U}$ and Gaussian Ansatz 26

5.2 Leading-twist $A_{L L}$ and first test of Gaussian Ansatz in polarized scattering 28

5.3 Leading-twist $A_{U T}^{\sin \left(\phi_{h}-\phi_{S}\right)}$ Sivers asymmetry $\quad 29$

5.4 Leading-twist $A_{U T}^{\sin \left(\phi_{h}+\phi_{S}\right)}$ Collins asymmetry 31

5.5 Leading-twist $A_{U U}^{\cos \left(2 \phi_{h}\right)}$ Boer-Mulders asymmetry 31

5.6 Leading-twist $A_{U T}^{\sin \left(3 \phi_{h}-\phi_{S}\right)}$ asymmetry 33

5.7 Statistical and systematic uncertainties of basis functions 33

$6 \quad$ Leading-twist asymmetries in WW-type approximation $\quad 34$

$\begin{array}{lll}6.1 & \text { Leading-twist } A_{L T}^{\cos \left(\phi_{h}-\phi_{S}\right)} \quad 35\end{array}$

6.2 Leading-twist $A_{U L}^{\sin 2 \phi_{h}}$ Kotzinian-Mulders asymmetry 36

$\begin{array}{lll}6.3 & \text { Inequalities and a cross check } & 37\end{array}$ 
7 Subleading-twist asymmetries in WW-type approximation

$\begin{array}{lll}7.1 & \text { Subleading-twist } A_{L U}^{\sin \phi_{h}} & 38\end{array}$

$\begin{array}{lll}7.2 & \text { Subleading-twist } A_{L T}^{\cos \phi_{S}} & 39\end{array}$

$\begin{array}{ll}7.3 & \text { Subleading-twist } A_{L T}^{\cos \left(2 \phi_{h}-\phi_{S}\right)}\end{array} 40$

$\begin{array}{ll}7.4 & \text { Subleading-twist } A_{L L}^{\cos \phi_{h}}\end{array} 41$

$\begin{array}{ll}\text { 7.5 } & \text { Subleading-twist } A_{U L}^{\sin \phi_{h}}\end{array}$

$\begin{array}{lll}7.6 & \text { Subleading-twist } A_{U T}^{\sin \phi_{S}} & 42\end{array}$

7.7 Subleading-twist $A_{U T}^{\sin \left(2 \phi_{h}-\phi_{S}\right)} 44$

$\begin{array}{ll}7.8 \text { Subleading-twist } A_{U U}^{\cos \phi_{h}} & 45\end{array}$

8 Conclusions $\quad 46$

A The "minimal basis" of TMDs and FFs 48

A.1 Unpolarized functions $f_{1}^{a}\left(x, k_{\perp}^{2}\right)$ and $D_{1}^{a}\left(z, P_{\perp}^{2}\right)$

$\begin{array}{lll}\text { A.2 Helicity distribution } g_{1}^{a}\left(x, k_{\perp}^{2}\right) & 49\end{array}$

A.3 Sivers function $f_{1 T}^{\perp q}\left(x, k_{\perp}^{2}\right) \quad 50$

A.4 Transversity $h_{1}^{q}\left(x, k_{\perp}^{2}\right)$ and Collins function $H_{1}^{\perp q}\left(x, P_{\perp}^{2}\right)$

A.5 Boer-Mulders function $h_{1}^{\perp q}\left(x, k_{\perp}^{2}\right) \quad 52$

A.6 Pretzelosity distribution $h_{1 T}^{\perp q}\left(x, k_{\perp}^{2}\right)$

B Convolution integrals and expressions in Gaussian Ansatz 53

B.1 Notation for convolution integrals $\quad 53$

B.2 Gaussian Ansatz 54

B.3 Gaussian Ansatz for the derived TMDs used in this work 55

B.4 Comment on TMDs subject to the sum rules (2.13) 55

B.5 Convolution integrals in Gaussian Ansatz 56

\section{Introduction}

A great deal of what is known about the quark-gluon structure of nucleons is due to studies of parton distribution functions (PDFs) in deep-inelastic reactions. Leading-twist PDFs tell us how likely it is to find an unpolarized parton [described by $\operatorname{PDF} f_{1}^{a}(x)$, $a=q, \bar{q}, g$ ] or a longitudinally polarized parton [described by $\operatorname{PDF} g_{1}^{a}(x), a=q, \bar{q}, g$ ] in a fast-moving unpolarized or longitudinally polarized nucleon, which carries the fraction $x$ of the nucleon momentum. This information depends on the "resolution (renormalization) scale" associated with the hard scale $Q$ of the process. Although the PDFs $f_{1}^{a}(x)$ and $g_{1}^{a}(x)$ continue being the subject of intense research (small- $x$, large- $x$, helicity sea and gluon distributions) they can be considered as rather well known, and the frontier has been extended in the last years to go beyond the one-dimensional picture offered by those PDFs.

One way to do this consists in a systematic inclusion of transverse parton momenta $k_{\perp}$, whose effects manifest themselves in terms of transverse momenta of the reaction 
products in the final state. If these transverse momenta are much smaller than the hard scale $Q$ of the process, the formal description is given in terms of transverse momentum dependent distribution functions (TMDs) and fragmentation functions (FFs), which are defined in terms of quark-quark correlators [1-5]. Both of them depend on two independent variables: in the case of TMDs, on the fraction $x$ of nucleon momentum carried by the parton and intrinsic transverse momentum $k_{\perp}$ of the parton, while in the case of FFs, on the fraction $z$ of the parton momentum transferred to the hadron and the transverse momentum of the hadron acquired during the fragmentation process. Being a vector in the plane transverse with respect to the light-cone direction singled out by the hard-momentum flow in the process, $k_{\perp}$ allows us to access novel information on the nucleon spin structure through correlations of $k_{\perp}$ with the nucleon and/or parton spin. The latter is a well-defined concept for twist-2 TMDs interpreted in the infinite momentum frame or in the lightcone quantization formalism.

One powerful tool to study TMDs are measurements of the semi-inclusive deep-inelastic scattering (SIDIS) process. By exploring various possibilities for the lepton beam and target polarizations unambiguous information can be accessed on the 8 leading-twist TMDs [3] and, if one assumes factorization, on certain linear combinations of the 16 subleading-twist TMDs $[4,5]$. It is important to stress that this information could not have been obtained without advances in target polarization techniques employed in the HERMES, COMPASS and Jefferson Lab (JLab) experiments [6-9]. Complementary information can be obtained from the Drell-Yan process [10], and $e^{+} e^{-}$annihilation [11].

In QCD the TMDs are independent functions. Each TMD contains unique information on a different aspect of the nucleon structure. Twist-2 TMDs have partonic interpretations. Twist-3 TMDs give insights on quark-gluon correlations in the nucleon [12-14]. Besides positivity constraints [15] there is little model-independent information on TMDs. An important question with practical applications is: do useful approximations for TMDs exist? Experience from collinear PDFs encourages to explore this possibility: the twist$3 g_{T}^{a}(x)$ and $h_{L}^{a}(x)$ can be respectively expressed in terms of contributions from twist-2 $g_{1}^{a}(x)$ and $h_{1}^{a}(x)$, and additional quark-gluon-quark $(\bar{q} g q)$ correlations or current-quark mass terms $[16,17]$ (the index $a=q, \bar{q}$ does not include gluons for $h_{1}^{a}, h_{L}^{a}$ and other chiralodd TMDs below). We shall refer to the latter generically as $\bar{q} g q$-terms, keeping in mind one deals in each case with matrix elements of different operators. The $\bar{q} g q$-correlations contain new insights on hadron structure, which are worthwhile exploring for their own sake, see for instance ref. [18] on $g_{T}^{a}(x)$.

The striking observation is that the $\bar{q} g q$-terms in $g_{T}^{a}(x)$ and $h_{L}^{a}(x)$ are small: theoretical mechanisms predict this [19-22], and in the case of $g_{T}^{a}(x)$ data confirm or are compatible with these predictions [23-25]. This approximation ("neglect of $\bar{q} g q$-terms") is commonly known as Wandzura-Wilczek (WW) approximation [16]. The possibility to apply this type of approximation also to TMDs has been explored in specific cases in [26-32]. In both cases, PDFs and TMDs, one basically assumes that the contributions from $\bar{q} g q-$ terms can be neglected with respect to $\bar{q} q$-terms. But the nature of the omitted matrix elements is different, and in the context of TMDs one often prefers to speak about WW-type approximations. 
The WW-type approximation is not preserved under $Q^{2}$ evolution. Some intuition can be obtained from the collinear case. However, much less is known about the $k_{\perp}$-evolution especially at subleading twist. More theoretical work is required here.

The present work is the first study of all SIDIS structure functions up to twist-3 evaluated within one common systematic theoretical guideline. Our results are of importance for measurements performed or in preparation at COMPASS, HERMES, and JLab with $12 \mathrm{GeV}$ beam-energy upgrade, or proposed in the long-term (Electron-Ion Collider), and provide helpful input for the development of Monte Carlo event generators [33].

On the theoretical side it is also important to note that the theory for subleading-twist TMD observables is only poorly developed as compared to the current state-of-the-art of leading-twist observables. In order to address subleading-twist TMD observables one has to restrict oneself to the tree-level formalism [1-5], which may not be free of doubts [34, 35].

Our predictions, whether confirmed or not supported by current and future experimental data, will in any case provide a useful benchmark, and call for dedicated theoretical studies to explain (i) why the pertinent $\bar{q} g q$-terms are small or (ii) why they are sizable, depending on the outcome of the experiments. In either case our results will deepen the understanding of $\bar{q} g q$-correlations, pave the way towards testing the validity of the TMD factorization approach at subleading twist, and help us to guide further developments.

In this work, after introducing the SIDIS process and defining TMDs and FFs (section 2), we shall introduce the WW(-type) approximations, and review what is presently known about them from experiment and theory (section 3). We will show that under the assumption of the validity of these approximations all leading and subleading SIDIS structure functions are described in terms of a basis of 6 TMDs and 2 FFs (section 4), and review how these basis functions describe available data (section 5 ). We will systematically apply the WW and/or WW-type approximations to SIDIS structure functions at leading (section 6) and subleading (section 7) twist, and conclude with a critical discussion (section 8). The appendices A and B contain technical details. An open-source package is available which allows one to visualize and reproduce the results presented in this work, and may easily be adapted by interested colleagues for their purposes. ${ }^{1}$

\section{The SIDIS process in terms of TMDs and FFs}

In this section we review the description of the SIDIS process, define structure functions, PDFs, TMDs, FFs and recall how they describe the SIDIS structure functions.

\subsection{The SIDIS process}

The SIDIS process $l N \rightarrow l^{\prime} h X$ is sketched in figure 1 . Here, $l$ and $P$ are the momenta of the incoming lepton and nucleon, $l^{\prime}$ and $P_{h}$ are the momenta of the outgoing lepton and produced hadron. The virtual-photon momentum $q=l-l^{\prime}$ defines the z-axis, and $l^{\prime}$

\footnotetext{
${ }^{1}$ Open-source packages with implementations of SIDIS structure functions in the WW-type approximation are publicly available on github.com: in Mathematica, Version 11.3 on https://github.com/prokudin/ WW-SIDIS, in Python on https://jeffersonlab.github.io/jam3d.
} 


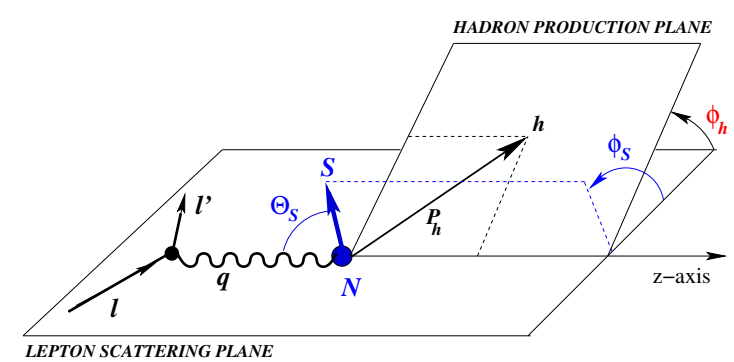

Figure 1. Kinematics of the SIDIS process $l N \rightarrow l^{\prime} h X$ in the 1-photon exchange approximation.

points in the direction of the $\mathrm{x}$-axis from which azimuthal angles are counted. The relevant kinematic invariants are

$$
x=\frac{Q^{2}}{2 P \cdot q}, \quad y=\frac{P \cdot q}{P \cdot l}, z=\frac{P \cdot P_{h}}{P \cdot q}, Q^{2}=-q^{2} .
$$

Note that we consider the production of unpolarized hadrons in DIS of charged leptons (electrons, positrons, muons) at $Q^{2} \ll M_{Z}^{2}$ in the single-photon exchange approximation, where $M_{Z}$ denotes the mass of the $Z^{0}$ electroweak gauge boson. In addition to $x, y$, and $z$, the cross section is also differential in the azimuthal angle $\phi_{h}$ of the produced hadron and in the square of the hadron's momentum component $P_{h T}$ perpendicular with respect to the virtual-photon momentum. The cross section is also differential with respect to the azimuthal angle $\psi_{l}$ characterizing the overall orientation of the lepton scattering plane around the incoming lepton direction. The angle is calculated with respect to an arbitrary reference axis, which in case of transversely polarized targets is chosen to be the transverse component $S_{T}$ of the target-spin direction. In the DIS limit, $\psi_{l} \approx \phi_{S}$, where the latter is the azimuthal angle of the spin-vector defined as in figure 1.

To leading order in $1 / Q$ the SIDIS cross-section is given by

$$
\begin{aligned}
& \frac{d^{6} \sigma_{\text {leading }}}{d x d y d z d \psi_{l} d \phi_{h} d P_{h T}^{2}}=\frac{\alpha_{e m}^{2}}{x y Q^{2}}\left(1-y+\frac{1}{2} y^{2}\right) F_{U U}\left(x, z, P_{h T}^{2}\right) \\
& \times\left\{1+\cos \left(2 \phi_{h}\right) p_{1} A_{U U}^{\cos \left(2 \phi_{h}\right)}+S_{L} \sin \left(2 \phi_{h}\right) p_{1} A_{U L}^{\sin \left(2 \phi_{h}\right)}+\lambda S_{L} p_{2} A_{L L}\right. \\
& +S_{T} \sin \left(\phi_{h}-\phi_{S}\right) A_{U T}^{\sin \left(\phi_{h}-\phi_{S}\right)}+S_{T} \sin \left(\phi_{h}+\phi_{S}\right) p_{1} A_{U T}^{\sin \left(\phi_{h}+\phi_{S}\right)} \\
& \left.+S_{T} \sin \left(3 \phi_{h}-\phi_{S}\right) p_{1} A_{U T}^{\sin \left(3 \phi_{h}-\phi_{S}\right)}+\lambda S_{T} \cos \left(\phi_{h}-\phi_{S}\right) p_{2} A_{L T}^{\cos \left(\phi_{h}-\phi_{S}\right)}\right\} \text {. }
\end{aligned}
$$

Here $F_{U U}$ is the structure function due to transverse polarization of the virtual photon (sometimes denoted as $F_{U U, T}$ ), and we neglect $1 / Q^{2}$ corrections in kinematic factors and a structure function (sometimes denoted as $F_{U U, L}$ ) arising from longitudinal polarization of the virtual photon (and another structure function $\propto S_{T} \sin \left(\phi_{h}-\phi_{S}\right)$, see below). The structure functions (and asymmetries) also depend on $Q^{2}$ via the scale dependence of TMDs and FFs, which we do not show in formulas throughout this work. 
At subleading order in the $1 / Q$ expansion one has

$$
\begin{aligned}
& \frac{d^{6} \sigma_{\text {subleading }}}{d x d y d z d \psi_{l} d \phi_{h} d P_{h T}^{2}}=\frac{\alpha_{e m}^{2}}{x y Q^{2}}\left(1-y+\frac{1}{2} y^{2}\right) F_{U U}\left(x, z, P_{h T}^{2}\right)\left\{\cos \left(\phi_{h}\right) p_{3} A_{U U}^{\cos \left(\phi_{h}\right)}\right. \\
& \quad+\lambda \sin \left(\phi_{h}\right) p_{4} A_{L U}^{\sin \left(\phi_{h}\right)}+S_{L} \sin \left(\phi_{h}\right) p_{3} A_{U L}^{\sin \left(\phi_{h}\right)}+\lambda S_{L} \cos \left(\phi_{h}\right) p_{4} A_{L L}^{\cos \left(\phi_{h}\right)} \\
& \quad+S_{T} \sin \left(2 \phi_{h}-\phi_{S}\right) p_{3} A_{U T}^{\sin \left(2 \phi_{h}-\phi_{S}\right)}+S_{T} \sin \left(\phi_{S}\right) p_{3} A_{U T}^{\sin \left(\phi_{S}\right)} \\
& \left.\quad+\lambda S_{T} \cos \left(\phi_{S}\right) p_{4} A_{L T}^{\cos \left(\phi_{S}\right)}+\lambda S_{T} \cos \left(2 \phi_{h}-\phi_{S}\right) p_{4} A_{L T}^{\cos \left(2 \phi_{h}-\phi_{S}\right)}\right\}
\end{aligned}
$$

Neglecting $1 / Q^{2}$ corrections, the kinematic prefactors $p_{i}$ are given by

$$
p_{1}=\frac{1-y}{1-y+\frac{1}{2} y^{2}}, \quad p_{2}=\frac{y\left(1-\frac{1}{2} y\right)}{1-y+\frac{1}{2} y^{2}}, \quad p_{3}=\frac{(2-y) \sqrt{1-y}}{1-y+\frac{1}{2} y^{2}}, \quad p_{4}=\frac{y \sqrt{1-y}}{1-y+\frac{1}{2} y^{2}},
$$

and the asymmetries $A_{X Y}^{\text {weight }}$, are defined in terms of structure functions $F_{X Y}^{\text {weight }}$, as follows

$$
A_{X Y}^{\text {weight }} \equiv A_{X Y}^{\text {weight }}\left(x, z, P_{h T}\right)=\frac{F_{X Y}^{\text {weight }}\left(x, z, P_{h T}\right)}{F_{U U}\left(x, z, P_{h T}\right)} .
$$

Here, the first subscript $X=U(L)$ denotes the unpolarized beam (longitudinally polarized beam with helicity $\lambda)$. The second subscript $Y=U(L$ or $T)$ refers to the target, which can be unpolarized (longitudinally or transversely polarized with respect to the virtual photon). The superscript "weight" indicates the azimuthal dependence with no index indicating a $\phi_{h}$-independent asymmetry or structure function.

In the partonic description the structure functions in (2.2a) are "twist-2." Those in (2.2b) are "twist-3" and contain a factor $M_{N} / Q$ in their definitions, see below, where $M_{N}$ is the nucleon mass. In our treatment to $1 / Q^{2}$ accuracy we neglect two structure functions due to longitudinal virtual-photon polarization, which contribute at order $\mathcal{O}\left(M_{N}^{2} / Q^{2}\right)$ in the partonic description of the process, one being $F_{U U, L}$ and the other contributing to the $\sin \left(\phi_{h}-\phi_{S}\right)$ angular distribution [5].

Experimental collaborations often define asymmetries in terms of counts $N\left(\phi_{h}\right)$. This means the kinematic prefactors $p_{i}$ and $1 /\left(x y Q^{2}\right)$ are included in the numerators or denominators of the asymmetries which are averaged over $y$ within experimental kinematics. We will call the corresponding asymmetries $A_{X Y,\langle y\rangle}^{\text {weight }}$. For instance, in the unpolarized case one has

$$
N\left(x, \ldots, \phi_{h}\right)=\frac{N_{0}(x, \ldots)}{2 \pi}\left(1+\cos \phi_{h} A_{U U,\langle y\rangle}^{\cos \phi_{h}}(x, \ldots)+\cos 2 \phi_{h} A_{U U,\langle y\rangle}^{\cos 2 \phi_{h}}(x, \ldots)\right)
$$

where $N_{0}$ denotes the total ( $\phi_{h}$-averaged) number of counts and the dots indicate further kinematic variables in the kinematic bin of interest (which may also be averaged over). It would be preferable if asymmetries were analyzed with known kinematic prefactors divided out on event-by-event basis. One could then directly compare asymmetries $A_{X Y}^{\text {weight }}$ 
measured in different experiments and kinematics, and focus on effects of evolution or power suppression for twist-3. In practice, often the kinematic factors were included. We will define and comment on the explicit expressions as needed.

For completeness we remark that after integrating the cross section over transverse hadron momenta one obtains

$$
\begin{aligned}
\frac{d^{4} \sigma_{\text {leading }}}{d x d y d z d \psi_{l}}= & \frac{1}{2 \pi} \frac{4 \pi \alpha_{e m}^{2}}{x y Q^{2}}\left(1-y+\frac{1}{2} y^{2}\right) F_{U U}(x, z)\left\{1+\lambda S_{L} p_{2} A_{L L}\right\} \\
\frac{d^{4} \sigma_{\text {subleading }}}{d x d y d z d \psi_{l}}= & \frac{1}{2 \pi} \frac{4 \pi \alpha_{e m}^{2}}{x y Q^{2}}\left(1-y+\frac{1}{2} y^{2}\right) F_{U U}(x, z)\left\{S_{T} \sin \left(\phi_{S}\right) p_{3} A_{U T}^{\sin \left(\phi_{S}\right)}\right. \\
& \left.+\lambda S_{T} \cos \left(\phi_{S}\right) p_{4} A_{L T}^{\cos \left(\phi_{S}\right)}\right\}
\end{aligned}
$$

where (and analogous for the other structure functions)

$$
F_{U U}(x, z)=\int d^{2} P_{h T} F_{U U}\left(x, z, P_{h T}\right)
$$

and the asymmetries are defined as

$$
A_{X Y}^{\text {weight }}(x, z)=\frac{F_{X Y}^{\text {weight }}(x, z)}{F_{U U}(x, z)} .
$$

The connection of "collinear" SIDIS structure functions in (2.6a), (2.6b) to those known from inclusive DIS is established by integrating over $z$ and summing over hadrons as

$$
\begin{aligned}
\sum_{h} \int d z z F_{U U}(x, z) & \equiv 2 x F_{1}(x), \\
\sum_{h} \int d z z F_{L L}(x, z) & \equiv 2 x g_{1}(x) \\
\sum_{h} \int d z z F_{L T}^{\cos \phi_{S}}(x, z) \equiv-\gamma 2 x\left(g_{1}(x)+g_{2}(x)\right), & 0, \\
\sum_{h} \int d z z F_{U T}^{\sin \phi_{S}}(x, z) & =
\end{aligned}
$$

where $\gamma=2 M_{N} x / Q$ signals the twist-3 character of $F_{L T}^{\cos \phi_{S}}(x, z)$. Notice that $F_{U T}^{\sin \phi_{S}}(x, z)$ has no DIS counterpart due to time-reversal symmetry of strong interactions, and terms suppressed by $1 / Q^{2}$ are consequently neglected throughout this work including the twist- 4 DIS structure function $F_{L}(x)$.

\subsection{TMDs, FFs and structure functions}

TMDs are defined in terms of light-front correlators

$$
\Phi\left(x, \boldsymbol{k}_{\perp}\right)_{i j}=\left.\int \frac{d \xi^{-} d^{2} \boldsymbol{\xi}_{\perp}}{(2 \pi)^{3}} e^{i k \xi}\left\langle N(P, S)\left|\bar{\psi}_{j}(0) \mathcal{W}_{(0, \infty)} \mathcal{W}_{(\infty, \xi)} \psi_{i}(\xi)\right| N(P, S)\right\rangle\right|_{\begin{array}{l}
\xi^{+}=0 \\
k^{+}=x P^{+}
\end{array}}
$$


where the Wilson lines $\mathcal{W}_{(0, \infty)} \mathcal{W}_{(\infty, \xi)}$ refer to the SIDIS process [36]. For a generic fourvector $a^{\mu}$ we define the light-cone coordinates $a^{\mu}=\left(a^{+}, a^{-}, a_{\perp}\right)$ with $a^{ \pm}=\left(a^{0} \pm a^{3}\right) / \sqrt{2}$. The light-cone direction is singled out by the virtual-photon momentum and transverse vectors like $\boldsymbol{k}_{\perp}$ are perpendicular to it. In the virtual-photon-nucleon center-of-mass frame, the nucleon and the partons inside it move in the $(+)$-lightcone direction, while the struck quark and the produced hadron move in the (-)-light-cone direction. In the nucleon rest frame the polarization vector is given by $S=\left(0, \boldsymbol{S}_{T}, S_{L}\right)$ with $\boldsymbol{S}_{T}^{2}+S_{L}^{2}=1$.

The 8 leading-twist TMDs [3] are projected out from the correlator (2.10) as follows (blue: T-even TMDs, red: T-odd TMDs; all TMDs depend on $x, k_{\perp}$, renormalization scale and carry a flavor index which we do not indicate for brevity):

$$
\begin{aligned}
\frac{1}{2} \operatorname{Tr}\left[\gamma^{+} \Phi\left(x, \boldsymbol{k}_{\perp}\right)\right] & =f_{1}-\frac{\varepsilon^{j k} k_{\perp}^{j} S_{T}^{k}}{M_{N}} f_{1 T}^{\perp}, \\
\frac{1}{2} \operatorname{Tr}\left[\gamma^{+} \gamma_{5} \Phi\left(x, \boldsymbol{k}_{\perp}\right)\right] & =S_{L} g_{1}+\frac{\boldsymbol{k}_{\perp} \cdot \boldsymbol{S}_{T}}{M_{N}} g_{1 T}^{\perp}, \\
\frac{1}{2} \operatorname{Tr}\left[i \sigma^{j+} \gamma_{5} \Phi\left(x, \boldsymbol{k}_{\perp}\right)\right] & =S_{T}^{j} h_{1}+S_{L} \frac{k_{\perp}^{j}}{M_{N}} h_{1 L}^{\perp}+\frac{\kappa^{j k} S_{T}^{k}}{M_{N}^{2}} h_{1 T}^{\perp}+\frac{\varepsilon^{j k} k_{\perp}^{k}}{M_{N}} h_{1}^{\perp},
\end{aligned}
$$

and the 16 subleading-twist TMDs $[2,5]$ are given by

$$
\begin{aligned}
\frac{1}{2} \operatorname{Tr}\left[1 \Phi\left(x, \boldsymbol{k}_{\perp}\right)\right] & =\frac{M_{N}}{P^{+}}\left[e-\frac{\varepsilon^{j k} k_{\perp}^{j} S_{T}^{k}}{M_{N}} e_{T}^{\perp}\right] \\
\frac{1}{2} \operatorname{Tr}\left[i \gamma_{5} \Phi\left(x, \boldsymbol{k}_{\perp}\right)\right] & =\frac{M_{N}}{P^{+}}\left[S_{L} e_{L}+\frac{\boldsymbol{k}_{\perp} \cdot \boldsymbol{S}_{T}}{M_{N}} e_{T}\right] \\
\frac{1}{2} \operatorname{Tr}\left[\gamma^{j} \Phi\left(x, \boldsymbol{k}_{\perp}\right)\right] & =\frac{M_{N}}{P^{+}}\left[\frac{k_{\perp}^{j}}{M_{N}} f^{\perp}+\varepsilon^{j k} S_{T}^{k} f_{T}+S_{L} \frac{\varepsilon^{j k} k_{\perp}^{k}}{M_{N}} f_{L}^{\perp}-\frac{\kappa^{j k} \varepsilon^{k l} S_{T}^{l}}{M_{N}^{2}} f_{T}^{\perp}\right], \\
\frac{1}{2} \operatorname{Tr}\left[\gamma^{j} \gamma_{5} \Phi\left(x, \boldsymbol{k}_{\perp}\right)\right] & =\frac{M_{N}}{P^{+}}\left[S_{T}^{j} g_{T}+S_{L} \frac{k_{\perp}^{j}}{M_{N}} g_{L}^{\perp}+\frac{\kappa^{j k} S_{T}^{k}}{M_{N}^{2}} g_{T}^{\perp}+\frac{\varepsilon^{j k} k_{\perp}^{k}}{M_{N}} g^{\perp}\right], \\
\frac{1}{2} \operatorname{Tr}\left[i \sigma^{j k} \gamma_{5} \Phi\left(x, \boldsymbol{k}_{\perp}\right)\right] & =\frac{M_{N}}{P^{+}}\left[\frac{S_{T}^{j} k_{\perp}^{k}-S_{T}^{k} k_{\perp}^{j}}{M_{N}} h_{T}^{\perp}-\varepsilon^{j k} h\right] \\
\frac{1}{2} \operatorname{Tr}\left[i \sigma^{+-} \gamma_{5} \Phi\left(x, \boldsymbol{k}_{\perp}\right)\right] & =\frac{M_{N}}{P^{+}}\left[S_{L} h_{L}+\frac{\boldsymbol{k}_{\perp} \cdot \boldsymbol{S}_{T}}{M_{N}} h_{T}\right]
\end{aligned}
$$

where $\kappa^{j k} \equiv\left(k_{\perp}^{j} k_{\perp}^{k}-\frac{1}{2} \boldsymbol{k}_{\perp}^{2} \delta^{j k}\right)$. The indices $j, k, l$ refer to the plane transverse with respect to the light cone, $\epsilon^{i j} \equiv \epsilon^{-+i j}$ and $\epsilon^{0123}=+1$. Dirac structures not listed in (2.11a)-(2.11i) are twist-4 [4]. Integrating out transverse momenta in the correlator (2.10) leads to the "usual" PDFs known from collinear kinematics [17, 37], namely at twist-2 level

$$
\begin{aligned}
\frac{1}{2} \operatorname{Tr}\left[\gamma^{+} \Phi(x)\right] & =f_{1}, \\
\frac{1}{2} \operatorname{Tr}\left[\gamma^{+} \gamma_{5} \Phi(x)\right] & =S_{L} g_{1}, \\
\frac{1}{2} \operatorname{Tr}\left[i \sigma^{j+} \gamma_{5} \Phi(x)\right] & =S_{T}^{j} h_{1},
\end{aligned}
$$


and at twist-3 level

$$
\begin{aligned}
\frac{1}{2} \operatorname{Tr}[1 \Phi(x)] & =\frac{M_{N}}{P^{+}} e, \\
\frac{1}{2} \operatorname{Tr}\left[\gamma^{j} \gamma_{5} \Phi(x)\right] & =\frac{M_{N}}{P^{+}} S_{T}^{j} g_{T}, \\
\frac{1}{2} \operatorname{Tr}\left[i \sigma^{+-} \gamma_{5} \Phi(x)\right] & =\frac{M_{N}}{P^{+}} S_{L} h_{L} .
\end{aligned}
$$

Other structures drop out either due to explicit $k_{\perp}$-dependence, or due to the sum rules [5]

$$
\int d^{2} \boldsymbol{k}_{\perp} f_{T}^{a}\left(x, k_{\perp}^{2}\right)=\int d^{2} \boldsymbol{k}_{\perp} e_{L}^{a}\left(x, k_{\perp}^{2}\right)=\int d^{2} \boldsymbol{k}_{\perp} h^{a}\left(x, k_{\perp}^{2}\right)=0
$$

imposed by time reversal constraints.

Fragmentation functions are defined through the following correlator [11] (where $\boldsymbol{P}_{\perp}$ denotes the transverse momentum of the produced hadrons acquired during the fragmentation process with respect to the quark):

$$
\Delta\left(z, \boldsymbol{P}_{\perp}\right)_{i j}=\sum_{X} \int \frac{d \xi^{+} d^{2} \boldsymbol{\xi}_{\perp}}{2 z(2 \pi)^{3}} e^{i p \xi}\left\langle 0\left|\mathcal{W}_{(\infty, \xi)} \psi_{i}(\xi)\right| h, X\right\rangle\left\langle h, X\left|\bar{\psi}_{j}(0) \mathcal{W}_{(0, \infty)}\right| 0\right\rangle \mid \begin{aligned}
& \xi^{-}=0 \\
& p^{-}=P_{h}^{-} / z \\
& \boldsymbol{p}_{\perp}=-\boldsymbol{P}_{\perp} / z
\end{aligned}
$$

In this work we will consider only unpolarized final-state hadrons. If the produced hadron moves fast in the (-) light-cone direction, the twist-2 FFs are projected out as

$$
\begin{aligned}
\frac{1}{2} \operatorname{Tr}\left[\gamma^{-} \Delta\left(z, \boldsymbol{P}_{\perp}\right)\right] & =D_{1}, \\
\frac{1}{2} \operatorname{Tr}\left[i \sigma^{j-} \gamma_{5} \Delta\left(z, \boldsymbol{P}_{\perp}\right)\right] & =\epsilon^{j k} \frac{P_{\perp}^{k}}{z m_{h}} H_{1}^{\perp},
\end{aligned}
$$

and at twist-3 level

$$
\begin{aligned}
\frac{1}{2} \operatorname{Tr}\left[1 \Delta\left(z, \boldsymbol{P}_{\perp}\right)\right] & =\frac{m_{h}}{P_{h}^{-}} E, \\
\frac{1}{2} \operatorname{Tr}\left[\gamma^{j} \Delta\left(z, \boldsymbol{P}_{\perp}\right)\right] & =-\frac{P_{\perp}^{j}}{z P_{h}^{-}} D^{\perp}, \\
\frac{1}{2} \operatorname{Tr}\left[\gamma^{j} \gamma_{5} \Delta\left(z, \boldsymbol{P}_{\perp}\right)\right] & =\varepsilon^{j k} \frac{P_{\perp}^{k}}{z P_{h}^{-}} G^{\perp}, \\
\frac{1}{2} \operatorname{Tr}\left[i \sigma^{j k} \gamma_{5} \Delta\left(z, \boldsymbol{P}_{\perp}\right)\right] & =-\varepsilon^{j k} \frac{m_{h}}{P_{h}^{-}} H .
\end{aligned}
$$

The FFs depend on $z, P_{\perp}$, renormalization scale, quark flavor and type of hadron which we do not indicate for brevity. Integration over transverse hadron momenta leaves us with $D_{1}(z), E(z), H(z)$ while the other structures drop out due to their $P_{\perp}$ dependence. 
The structure functions in eqs. (2.2a), (2.2b) are described in the Bjorken limit at tree level in terms of convolutions of TMDs and FFs. We define the unit vector $\hat{\boldsymbol{h}}=\boldsymbol{P}_{h T} / P_{h T}$ and use the following convolution integrals (see appendix B.1 for details)

$$
\mathcal{C}[\omega f D]=x \sum_{a} e_{a}^{2} \int d^{2} \boldsymbol{k}_{\perp} d^{2} \boldsymbol{P}_{\perp} \delta^{(2)}\left(z \boldsymbol{k}_{\perp}+\boldsymbol{P}_{\perp}-\boldsymbol{P}_{h T}\right) \omega f^{a}\left(x, \boldsymbol{k}_{\perp}^{2}\right) D^{a}\left(z, \boldsymbol{P}_{\perp}^{2}\right)
$$

where $\omega$ is a weight function, which in general depends on $\boldsymbol{k}_{\perp}$ and $\boldsymbol{P}_{\perp}$. The 8 leading-twist structure functions are

$$
\begin{aligned}
F_{U U} & =\mathcal{C}\left[\omega^{\{0\}} f_{1} D_{1}\right], \\
F_{U U}^{\cos 2 \phi_{h}} & =\mathcal{C}\left[\omega_{\mathrm{AB}}^{\{2\}} h_{1}^{\perp} H_{1}^{\perp}\right] \\
F_{U L}^{\sin 2 \phi_{h}} & =\mathcal{C}\left[\omega_{\mathrm{AB}}^{\{2\}} h_{1 L}^{\perp} H_{1}^{\perp}\right], \\
F_{L L} & =\mathcal{C}\left[\omega^{\{0\}} g_{1} D_{1}\right] \\
F_{L T}^{\cos \left(\phi_{h}-\phi_{S}\right)} & =\mathcal{C}\left[\omega_{\mathrm{B}}^{\{1\}} g_{1 T}^{\perp} D_{1}\right] \\
F_{U T}^{\sin \left(\phi_{h}+\phi_{S}\right)} & =\mathcal{C}\left[\omega_{\mathrm{A}}^{\{1\}} h_{1} H_{1}^{\perp}\right] \\
F_{U T}^{\sin \left(\phi_{h}-\phi_{S}\right)} & =\mathcal{C}\left[-\omega_{\mathrm{B}}^{\{1\}} f_{1 T}^{\perp} D_{1}\right], \\
F_{U T}^{\sin \left(3 \phi_{h}-\phi_{S}\right)} & =\mathcal{C}\left[\omega^{\{3\}} h_{1 T}^{\perp} H_{1}^{\perp}\right] .
\end{aligned}
$$

At subleading-twist we have the structure functions

$$
\begin{aligned}
F_{U U}^{\cos \phi_{h}}= & \frac{2 M_{N}}{Q} \mathcal{C}\left[\omega_{\mathrm{A}}^{\{1\}}\left(x h H_{1}^{\perp}+r_{h} f_{1} \frac{\tilde{D}^{\perp}}{z}\right)-\omega_{\mathrm{B}}^{\{1\}}\left(x f^{\perp} D_{1}+r_{h} h_{1}^{\perp} \frac{\tilde{H}}{z}\right)\right], \\
F_{L U}^{\sin \phi_{h}}= & \frac{2 M_{N}}{Q} \mathcal{C}\left[\omega_{\mathrm{A}}^{\{1\}}\left(x e H_{1}^{\perp}+r_{h} f_{1} \frac{\tilde{G}^{\perp}}{z}\right)+\omega_{\mathrm{B}}^{\{1\}}\left(x g^{\perp} D_{1}+r_{h} h_{1}^{\perp} \frac{\tilde{E}}{z}\right)\right], \\
F_{U L}^{\sin \phi_{h}}= & \frac{2 M_{N}}{Q} \mathcal{C}\left[\omega_{\mathrm{A}}^{\{1\}}\left(x h_{L} H_{1}^{\perp}+r_{h} g_{1} \frac{\tilde{G}^{\perp}}{z}\right)+\omega_{\mathrm{B}}^{\{1\}}\left(x f_{L}^{\perp} D_{1}-r_{h} h_{1 L}^{\perp} \frac{\tilde{H}}{z}\right)\right], \\
F_{L L}^{\cos \phi_{h}}= & \frac{2 M_{N}}{Q} \mathcal{C}\left[-\omega_{\mathrm{A}}^{\{1\}}\left(x e_{L} H_{1}^{\perp}-r_{h} g_{1} \frac{\tilde{D}^{\perp}}{z}\right)-\omega_{\mathrm{B}}^{\{1\}}\left(x g_{L}^{\perp} D_{1}+r_{h} h_{1 L}^{\perp} \frac{\tilde{E}}{z}\right)\right], \\
F_{U T}^{\sin \phi_{S}}= & \frac{2 M_{N}}{Q} \mathcal{C}\left[\omega^{\{0\}}\left(x f_{T} D_{1}-r_{h} h_{1} \frac{\tilde{H}}{z}\right)\right. \\
& \left.-\frac{\omega_{\mathrm{B}}^{\{2\}}}{2}\left(x h_{T} H_{1}^{\perp}+r_{h} g_{1 T}^{\perp} \frac{\tilde{G}^{\perp}}{z}-x h_{T}^{\perp} H_{1}^{\perp}+r_{h} f_{1 T}^{\perp} \frac{\tilde{D}^{\perp}}{z}\right)\right],
\end{aligned}
$$




$$
\begin{aligned}
& F_{L T}^{\cos \phi_{S}}=\frac{2 M_{N}}{Q} \mathcal{C}\left[-\omega^{\{0\}}\left(x g_{T} D_{1}+r_{h} h_{1} \frac{\tilde{E}}{z}\right)\right. \\
& \left.+\frac{\omega_{\mathrm{B}}^{\{2\}}}{2}\left(x e_{T} H_{1}^{\perp}-r_{h} g_{1 T}^{\perp} \frac{\tilde{D}^{\perp}}{z}+x e_{T}^{\perp} H_{1}^{\perp}+r_{h} f_{1 T}^{\perp} \frac{\tilde{G}^{\perp}}{z}\right)\right], \\
& F_{U T}^{\sin \left(2 \phi_{h}-\phi_{S}\right)}=\frac{2 M_{N}}{Q} \mathcal{C}\left[\frac{\omega_{\mathrm{AB}}^{\{2\}}}{2}\left(x h_{T} H_{1}^{\perp}+r_{h} g_{1 T}^{\perp} \frac{\tilde{G}^{\perp}}{z}+x h_{T}^{\perp} H_{1}^{\perp}-r_{h} f_{1 T}^{\perp} \frac{\tilde{D}^{\perp}}{z}\right)\right. \\
& \left.+\omega_{\mathrm{C}}^{\{2\}}\left(x f_{T}^{\perp} D_{1}-r_{h} h_{1 T}^{\perp} \frac{\tilde{H}}{z}\right)\right], \\
& F_{L T}^{\cos \left(2 \phi_{h}-\phi_{S}\right)}=\frac{2 M_{N}}{Q} \mathcal{C}\left[-\frac{\omega_{\mathrm{AB}}^{\{2\}}}{2}\left(x e_{T} H_{1}^{\perp}-r_{h} g_{1 T}^{\perp} \frac{\tilde{D}^{\perp}}{z}-x e_{T}^{\perp} H_{1}^{\perp}-r_{h} f_{1 T}^{\perp} \frac{\tilde{G}^{\perp}}{z}\right)\right. \\
& \left.-\omega_{\mathrm{C}}^{\{2\}}\left(x g_{T}^{\perp} D_{1}+r_{h} h_{1 T}^{\perp} \frac{\tilde{E}}{z}\right)\right],
\end{aligned}
$$

where $r_{h}=m_{h} / M_{N}$ and $F_{X Y}^{\text {weight }} \equiv F_{X Y}^{\text {weight }}\left(x, z, P_{h T}\right)$. The tilde-functions $\tilde{D}^{\perp}, \tilde{G}^{\perp}, \tilde{H}, \tilde{E}$ are defined in terms of $\bar{q} g q$-correlators, see section 3.2. The weight functions are defined as

$$
\begin{aligned}
& \omega^{\{0\}}=1, \omega_{\mathrm{B}}^{\{1\}}=\frac{\hat{\boldsymbol{h}} \cdot \boldsymbol{k}_{\perp}}{M_{N}}, \\
& \omega_{\mathrm{A}}^{\{1\}}=\frac{\hat{\boldsymbol{h}} \cdot \boldsymbol{P}_{\perp}}{z m_{h}}, \omega_{\mathrm{B}}^{\{2\}}=-\frac{\boldsymbol{P}_{\perp} \cdot \boldsymbol{k}_{\perp}}{z M_{N} m_{h}}, \quad \omega_{\mathrm{C}}^{\{2\}}=\frac{2\left(\hat{\boldsymbol{h}} \cdot \boldsymbol{k}_{\perp}\right)^{2}-\boldsymbol{k}_{\perp}^{2}}{2 M_{N}^{2}}, \\
& \omega_{\mathrm{A}}^{\{2\}}=\frac{2\left(\hat{\boldsymbol{h}} \cdot \boldsymbol{P}_{\perp}\right)\left(\hat{\boldsymbol{h}} \cdot \boldsymbol{k}_{\perp}\right)}{z M_{N} m_{h}}, \quad\left(\hat{\boldsymbol{h}} \cdot \boldsymbol{P}_{\perp}\right) \boldsymbol{k}_{\perp}^{2} \\
& \omega^{\{3\}}=\frac{4\left(\hat{\boldsymbol{h}} \cdot \boldsymbol{P}_{\perp}\right)\left(\hat{\boldsymbol{h}} \cdot \boldsymbol{k}_{\perp}\right)^{2}-2\left(\hat{\boldsymbol{h}} \cdot \boldsymbol{k}_{\perp}\right)\left(\boldsymbol{k}_{\perp} \cdot \boldsymbol{P}_{\perp}\right)-\left(z M_{N}^{2} m_{h}\right.}{2}
\end{aligned}
$$

and $\omega_{\mathrm{AB}}^{\{2\}}=\omega_{\mathrm{A}}^{\{2\}}+\omega_{\mathrm{B}}^{\{2\}}$. In $\omega_{i}^{\{n\}}$ the index $n=0,1,2,3$ indicates the (maximal) power $\left(P_{h T}\right)^{n}$ with which the corresponding contribution scales, and index $i$ (if any) distinguishes different types of contributions at the given order $n$. Notice that twist-3 structure functions in eqs. $(2.18 \mathrm{a})-(2.18 \mathrm{~h})$ contain an explicit factor $M_{N} / Q$. We also recall that we neglect two structure functions (denoted in [5] as $F_{U U, L}$ and $F_{U T, L}^{\sin \left(\phi_{h}-\phi_{S}\right)}$ ) due to longitudinal virtualphoton polarization, which are of order $\mathcal{O}\left(M^{2} / Q^{2}\right)$ in the TMD partonic description.

The structure functions that survive $P_{h T}$-integration of the SIDIS cross section in (2.6a), (2.6b) are associated with the trivial weights $\omega^{\{0\}}$ and expressed in terms of collinear PDFs and FFs as follows (here the sum rules (2.13) are used):

$$
\begin{aligned}
F_{U U}(x, z) & =x \sum_{a} e_{a}^{2} f_{1}^{a}(x) D_{1}^{a}(z), \\
F_{L L}(x, z) & =x \sum_{a} e_{a}^{2} g_{1}^{a}(x) D_{1}^{a}(z), \\
F_{L T}^{\cos \phi_{S}}(x, z) & =-\frac{2 M_{N}}{Q} x \sum_{a} e_{a}^{2}\left(x g_{T}^{a}(x) D_{1}^{a}(z)+r_{h} h_{1}^{a}(x) \frac{\tilde{E}^{a}(z)}{z}\right), \\
F_{U T}^{\sin \phi_{S}}(x, z) & =-\frac{2 m_{h}}{Q} x \sum_{a} e_{a}^{2} h_{1}^{a}(x) \frac{\tilde{H}^{a}(z)}{z} .
\end{aligned}
$$


Finally, integrating over $z$, summing over hadrons, and using the sum rules for the T-odd FFs, $\sum_{h} \int d z \tilde{E}^{a}(z)=0$ and $\sum_{h} \int d z \tilde{H}^{a}(z)=0$, we recover eqs. (2.9a)-(2.9d) and obtain for the DIS structure functions

$$
\begin{aligned}
& F_{1}(x)=\frac{1}{2} \sum_{a} e_{a}^{2} f_{1}^{a}(x), \\
& g_{1}(x)=\frac{1}{2} \sum_{a} e_{a}^{2} g_{1}^{a}(x), \\
& g_{2}(x)=\frac{1}{2} \sum_{a} e_{a}^{2} g_{T}^{a}(x)-g_{1}(x) .
\end{aligned}
$$

Before introducing the WW-type approximations in the next section, we would like to add a comment on TMD factorization: the partonic description of the leading-twist structure functions in (2.17) is based on factorization theorems [38-42]. In contrast to this, the partonic description of the subleading-twist structure functions in (2.18) is based on the assumption that the SIDIS cross section factorizes.

A lot of progress has been achieved in recent years in the theoretical understanding of leading-twist observables within the TMD framework, including definition, renormalization and evolution of leading-twist TMDs [43-46], next-to-leading order corrections within the TMD framework [47], and phenomenological fits with evolution [48, 49]. The matching of twist-2 collinear and TMD quantities was studied to next-to-leading and next-to-next-toleading order [50, 51]. The WW approximation has been used recently in ref. [52] to connect the twist-2 TMDs $f_{1 T}^{\perp}, g_{1 T}^{\perp}, h_{1}^{\perp}, h_{1 L}^{\perp}$ to certain higher-twist collinear matrix elements.

In contrast to this, the theory for subleading-twist TMD observables is only poorly developed. Still to the present day, the state-of-the-art approach to subleading-twist TMD observables is the one of refs. [1-5], based on a TMD tree-level formalism, which we adopt here. In fact, the results of refs. [34, 35] indicate doubts even in the tree-level formalism. Recently, an attempt was made to remedy these doubts [53]. Keeping in mind these "words of warning," still the formulas (2.18) are the best that theory has to offer currently. We may consider (2.18) as a model itself for the twist-3 SIDIS observables. We hope that the phenomenological approach based on WW-type approximations pursued in this work might lead to more insight into these observables, and eventually might trigger more theory efforts in the future.

\section{$3 \quad$ WW and WW-type approximations}

In this section we will define the approximations and review what is known about them. The basic idea of the approximations is simple. One uses QCD equations of motion to separate contributions from $\bar{q} q$-terms and $\bar{q} g q$-terms and assumes that the latter can be neglected with respect to the leading $\bar{q} q$-terms with a useful accuracy (here the $\langle\ldots\rangle$ denote symbolically the matrix elements which enter the definitions of TMDs or FFs):

$$
\left|\frac{\langle\bar{q} g q\rangle}{\langle\bar{q} q\rangle}\right| \ll 1
$$




\subsection{WW approximation for PDFs}

The WW approximation applies in principle to all twist-3 PDFs, eqs. (2.12d), (2.12e), (2.12f). It was established first for $g_{T}^{a}(x)$ [16], and later for $h_{L}^{a}(x)$ [17]. The situation of $e^{a}(x)$ is somewhat special, see below and the review [54].

The origin of the approximations is as follows. The operators defining $g_{T}^{a}(x)$ and $h_{L}^{a}(x)$ can be decomposed by means of QCD equations of motion in twist-2 parts, and pure twist-3 (interaction dependent) $\bar{q} g q$-terms and current-quark mass terms. We denote $\bar{q} g q$-terms and mass terms collectively and symbolically by functions with a tilde. ${ }^{2}$ Such decompositions are possible because $g_{T}^{a}(x)$ and $h_{L}^{a}(x)$ are "twist-3" not according to the "strict QCD definition" (twist $=$ mass dimension of associated local operator minus its spin). Rather they are classified according to the "working definition" of twist [55] (a function is "twist $t$ " if, in addition to overall kinematic prefactors, it contributes to cross sections in a partonic description suppressed by $(M / Q)^{t-2}$ where $M$ is a generic hadronic and $Q$ the hard scale). The two definitions coincide for twist-2 quantities, but higher-twist observables in general contain "contaminations" by leading twist.

In this way one obtains the decompositions and, if they apply, WW approximations [16, 17] (keep in mind here tilde terms contain pure twist-3 and current-quark mass terms)

$$
\begin{aligned}
g_{T}^{a}(x) & =\int_{x}^{1} \frac{d y}{y} g_{1}^{a}(y)+\tilde{g}_{T}^{a}(x) \stackrel{\mathrm{WW}}{\approx} \int_{x}^{1} \frac{d y}{y} g_{1}^{a}(y), \\
h_{L}^{a}(x) & =2 x \int_{x}^{1} \frac{d y}{y^{2}} h_{1}^{a}(y)+\tilde{h}_{L}^{a}(x) \stackrel{\mathrm{WW}}{\approx} 2 x \int_{x}^{1} \frac{d y}{y^{2}} h_{1}^{a}(y), \\
x e^{a}(x) & =\quad x \tilde{e}^{a}(x) \stackrel{\mathrm{WW}}{\approx} 0,
\end{aligned}
$$

where we included $e^{a}(x)$ which is a special case in the sense that it receives no twist-2 contribution. A prefactor of $x$ is provided in (3.2c) to cancel a $\delta(x)$-type singularity [54].

The relations (3.2a)-(3.2c) have been derived basically using operator product expansion techniques $[16,17]$. Notice that the operators defining $g_{T}^{a}$ and $h_{L}^{a}$ can also be decomposed within the TMD framework by means of a combination of relations derived from the QCD equations of motion and further constraint relations, called Lorentz-invariance relations (LIRs), into a twist-2 part, and dynamical twist-3 (interactions dependent) $\bar{q} g q$-terms and current-quark mass terms (see recent review [56] and references therein).

We will come back to (3.2a), (3.2b) and review the theoretical predictions and supporting experiments, but before we will introduce the WW-type approximations for TMDs and FFs.

\subsection{WW-type approximations for TMDs and FFs}

Analogous to WW approximations for PDFs discussed in section 3.1, also certain TMDs and FFs can be decomposed into twist-2 contributions and tilde terms. The latter may be assumed, in the spirit of (3.1), to be small. Hereby it is important to keep in mind that for

\footnotetext{
${ }^{2}$ In the literature it is customary to reserve the term "tilde terms" for matrix elements of $\bar{q} g q$ operators as done, e.g., in ref. [5]. For convenience in this work "tilde terms" refers to both $\bar{q} g q$ terms and current-quark mass terms as done, e.g., in [30].
} 
each TMD or FF one deals with different types of ("unintegrated") $\bar{q} g q$-correlations, and we prefer to refer to them as WW-type approximations.

In the T-even case one obtains the following approximations, ${ }^{3}$ where the terms on the left-hand-side are twist-3, those on the right-hand-side (if any) are twist-2,

$$
\begin{aligned}
& x e^{q}\left(x, k_{\perp}^{2}\right) \stackrel{\text { WW-type }}{\approx} 0, \\
& x f^{\perp q}\left(x, k_{\perp}^{2}\right) \stackrel{\text { WW-type }}{\approx} f_{1}^{q}\left(x, k_{\perp}^{2}\right), \\
& x g_{L}^{\perp q}\left(x, k_{\perp}^{2}\right) \stackrel{\text { WW-type }}{\approx} g_{1}^{q}\left(x, k_{\perp}^{2}\right), \\
& x g_{T}^{\perp q}\left(x, k_{\perp}^{2}\right) \stackrel{\text { WW-type }}{\approx} g_{1 T}^{\perp q}\left(x, k_{\perp}^{2}\right), \\
& x g_{T}^{q}\left(x, k_{\perp}^{2}\right) \stackrel{\text { WW-type }}{\approx} g_{1 T}^{\perp(1) q}\left(x, k_{\perp}^{2}\right), \\
& x h_{L}^{q}\left(x, k_{\perp}^{2}\right) \stackrel{\text { WW-type }}{\approx}-2 h_{1 L}^{\perp(1) q}\left(x, k_{\perp}^{2}\right), \\
& x h_{T}^{q}\left(x, k_{\perp}^{2}\right) \stackrel{\text { WW-type }}{\approx}-h_{1}^{q}\left(x, k_{\perp}^{2}\right)-h_{1 T}^{\perp(1) q}\left(x, k_{\perp}^{2}\right), \\
& x h_{T}^{\perp q}\left(x, k_{\perp}^{2}\right) \stackrel{\text { WW-type }}{\approx} h_{1}^{q}\left(x, k_{\perp}^{2}\right)-h_{1 T}^{\perp(1) q}\left(x, k_{\perp}^{2}\right) .
\end{aligned}
$$

In the T-odd case one obtains the approximations

$$
\begin{aligned}
& x e_{L}^{q}\left(x, k_{\perp}^{2}\right) \stackrel{\text { WW-type }}{\approx} 0, \\
& x e_{T}^{q}\left(x, k_{\perp}^{2}\right) \stackrel{\text { WW-type }}{\approx} 0, \\
& x e_{T}^{\perp q}\left(x, k_{\perp}^{2}\right) \stackrel{\text { WW-type }}{\approx} 0, \\
& x g^{\perp q}\left(x, k_{\perp}^{2}\right) \stackrel{\text { WW-type }}{\approx} 0, \\
& x f_{L}^{\perp q}\left(x, k_{\perp}^{2}\right) \stackrel{\text { WW-type }}{\approx} 0, \\
& x f_{T}^{\perp q}\left(x, k_{\perp}^{2}\right) \stackrel{\text { WW-type }}{\approx} f_{1 T}^{\perp q}\left(x, k_{\perp}^{2}\right), \\
& x f_{T}^{q}\left(x, k_{\perp}^{2}\right) \stackrel{\text { WW-type }}{\approx}-f_{1 T}^{\perp(1) q}\left(x, k_{\perp}^{2}\right), \\
& x h^{q}\left(x, k_{\perp}^{2}\right) \stackrel{\text { WW-type }}{\approx}-2 h_{1}^{\perp(1) q}\left(x, k_{\perp}^{2}\right) .
\end{aligned}
$$

The superscript "(1)" denotes the first transverse moment of TMDs defined generically as

$$
f^{(1)}\left(x, k_{\perp}^{2}\right)=\frac{k_{\perp}^{2}}{2 M^{2}} f\left(x, k_{\perp}^{2}\right), \quad f^{(1)}(x)=\int d^{2} k_{\perp} f^{(1)}\left(x, k_{\perp}^{2}\right) .
$$

Two very useful WW-type approximations follow from combining the WW approximations (3.2a), (3.2b) with the WW-type approximations (3.3e), (3.3f). The resulting relations

\footnotetext{
${ }^{3}$ Notice that ref. [5] uses four-vector notation for transverse vectors, while in this paper we always utilize two-vectors for transverse vectors, such that e.g. $\boldsymbol{k}_{\perp \text { our }}^{2}=-p_{T \text { ref. [5] }}^{2}$ and analog for other scalar products of transverse vectors. In our notation transverse vectors are never understood as four-vectors such that $\boldsymbol{k}_{\perp \text { our }}^{2} \equiv k_{\perp \text { our }}^{2}$.
} 
are the only WW-type relations applicable to twist-2 TMDs and are given by [2, 29, 32]:

$$
\begin{aligned}
& g_{1 T}^{\perp(1) a}(x) \stackrel{\text { WW-type }}{\approx} x \int_{x}^{1} \frac{d y}{y} g_{1}^{a}(y), \\
& h_{1 L}^{\perp(1) a}(x) \stackrel{\text { WW-type }}{\approx}-x^{2} \int_{x}^{1} \frac{d y}{y^{2}} h_{1}^{a}(y) .
\end{aligned}
$$

Some of the above WW-type approximations were discussed in [2, 26-32]. WW-relations for FFs are actually not needed: in eqs. (2.17), (2.18) either twist-2 FFs $D_{1}^{q}, H_{1}^{\perp q}$ enter or tilde FFs, as a consequence of how the azimuthal angles are defined [5]. For completeness we quote the WW-type approximations for FFs [5]

$$
\begin{aligned}
& E\left(z, P_{\perp}^{2}\right) \stackrel{\text { WW-type }}{\approx} 0, \\
& G^{\perp}\left(z, P_{\perp}^{2}\right) \stackrel{\text { WW-type }}{\approx} 0,
\end{aligned}
$$

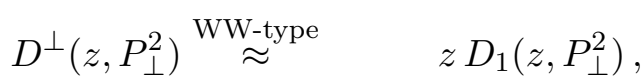

$$
\begin{aligned}
& H\left(z, P_{\perp}^{2}\right) \stackrel{\text { WW-type }}{\approx}-\frac{P_{\perp}^{2}}{z m_{h}^{2}} H_{1}^{\perp}\left(z, P_{\perp}^{2}\right) .
\end{aligned}
$$

Having introduced the WW and WW-type approximations, we will review in the following what is currently known from theory and experiment about the WW(-type) approximations.

\subsection{Predictions from instanton vacuum model}

Insights into the relative size of hadronic matrix elements, such as eq. (3.1), require a nonperturbative approach. It is by no means obvious which small parameter in the stronginteraction regime would allow one to explain such results. An appealing non-perturbative approach is provided by the instanton model of the QCD vacuum [57-59]. This semiclassical approach assumes that properties of the QCD vacuum are dominated by instantons and anti-instantons, topological non-perturbative gluon field configurations, which form a strongly interacting medium. The approach provides a natural mechanism for dynamical chiral-symmetry breaking, the dominant feature of strong interactions in the nonperturbative regime. It was shown with variational and numerical methods that the instantons form a dilute medium characterized by a non-trivial small parameter $\rho / R \sim 1 / 3$ [57-59], where $\rho$ and $R$ denote respectively the average instanton size $\rho$ and separation $R$.

Applying the instanton vacuum model to studies of $g_{T}^{a}(x)$ and $h_{L}^{a}(x)$, it was predicted that matrix elements of the $\bar{q} g q$ operators defining $\tilde{g}_{T}^{a}(x)[19]$ and $\tilde{h}_{L}^{a}(x)[20]$ are strongly suppressed by powers of the small parameter $\rho / R$ with respect to contributions from the respective twist-2 parts, which are of order $(\rho / R)^{0}$. For the $n=3$ Mellin moments (i.e. the lowest non-trivial ones for these tilde-functions) it was found $[19,20]$

$$
\frac{\tilde{g}_{T}^{q}}{g_{T}^{q}} \sim \frac{\tilde{h}_{L}^{q}}{h_{L}^{q}} \sim \frac{\langle\bar{q} g q\rangle}{\langle\bar{q} q\rangle} \sim\left(\frac{\rho}{R}\right)^{4} \log \left(\frac{\rho}{R}\right) \sim 10^{-2},
$$

which strongly supports the generic approximation in eq. (3.1) with the instanton packing fraction providing the non-trivial small parameter justifying the neglect of tilde terms. The 

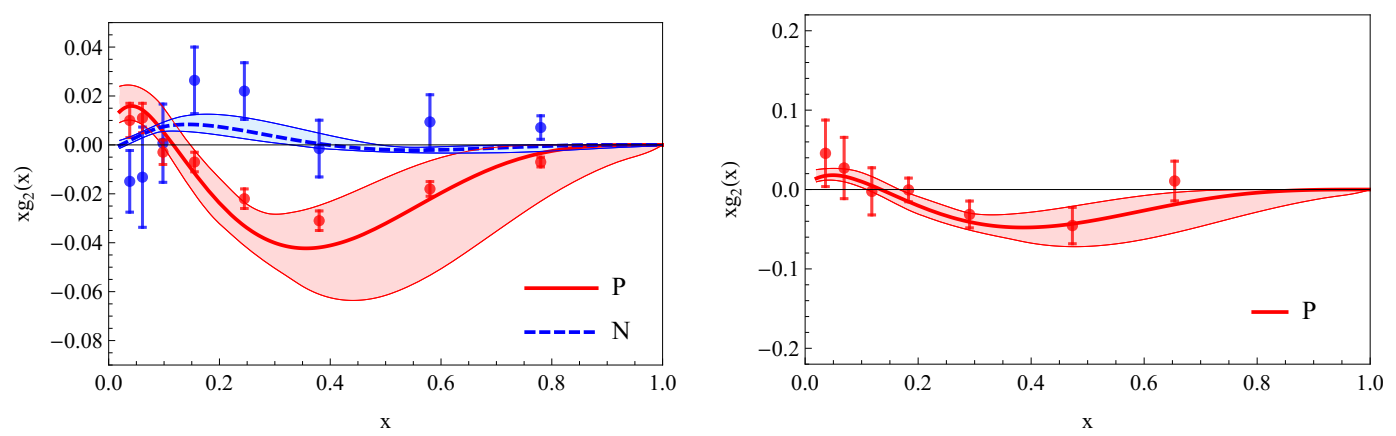

Figure 2. Left panel: the structure function $x g_{2}(x)$ in WW-approximation at $Q^{2}=7.1 \mathrm{GeV}^{2}$, eq. (3.9), for proton (P) and neutron (N) targets, and data from the SLAC E144 and E155 experiments at $\left\langle Q^{2}\right\rangle=7.1 \mathrm{GeV}^{2}[23,24]$. Right panel: HERMES data for $Q^{2}>1 \mathrm{GeV}^{2}$ with $\left\langle Q^{2}\right\rangle=2.4 \mathrm{GeV}^{2}[25]$. The estimate of the theoretical uncertainty is described in the text.

predictions for $\tilde{g}_{T}^{a}(x)$ [19] were made before the advent of the first precise data on $g_{2}(x)$, which we discuss next. The instanton calculus has not yet been applied to $\tilde{e}^{a}(x)$.

\subsection{Tests of WW approximation in DIS experiments}

The presently available phenomenological information on $g_{T}^{a}(x)$ is due to measurements of the structure function $g_{2}(x)$, eq. (2.21c), in DIS off various transversely polarized targets. In the WW-approximation (3.2a) one can write $g_{2}(x)$ as a total derivative expressed in terms of the experimentally well-known twist-2 structure function $g_{1}(x)$ as follows

$$
g_{2}(x) \stackrel{\mathrm{WW}}{\approx} g_{2}(x)_{\mathrm{WW}} \equiv \frac{d}{d x}\left[x \int_{x}^{1} \frac{d y}{y} g_{1}(y)\right] .
$$

Data support (3.9) to a good accuracy [23-25, 60], although especially at smaller $x$ more stringent tests are not yet possible. Overall it has been estimated that the WW approximation for $g_{2}(x)$ and $g_{T}^{a}(x)$ works with an accuracy of about $40 \%$ or better [61].

We present calculations of $g_{2}(x)_{\mathrm{Ww}}$ in figure 2. This result is obtained using the LO $g_{1}^{a}(x)$-parametrization [62]. In order to display the theoretical "uncertainty band" of this WW-approximation of about $40 \%$ as deduced in ref. [61] we proceed as follows: we split the $40 \%$ uncertainty into two parts: $\varepsilon_{1}= \pm 20 \%$ and $\varepsilon_{2}(x)= \pm 20 \%(1-x)^{\epsilon}$ with a small $\epsilon>0$, and estimate the impact of this uncertainty as

$$
g_{2}(x)_{\mathrm{WW}}=\left(1 \pm \varepsilon_{1}\right) \frac{d}{d x}\left[x \int_{x}^{1} \frac{d y}{y}\left(\frac{1}{2} \sum_{a} e_{a}^{2} g_{1}^{a}\left(y\left(1 \pm \varepsilon_{2}\right)\right)\right)\right] .
$$

The effect of $\varepsilon_{1}$ is to change the magnitude of $g_{2}(x)_{\mathrm{WW}}, \varepsilon_{2}$ varies the position of its zero. The $x$-dependence of $\varepsilon_{2}$ preserves $\lim _{x \rightarrow 1} g_{2}(x)=0$; we use $\epsilon=0.05$, which yields $\varepsilon_{2} \approx 20 \%$ up to the highest measured $x$-bin. The good agreement of $g_{2}(x)_{\mathrm{WW}}$ with data is encouraging, and in line with theory predictions [19]. Our estimate with the split uncertainties $\varepsilon_{1,2}$ may overestimate in certain $x$-bins the $40 \%$ - "uncertainty band" estimated in [61]. This however helps us to display a conservative estimate of possible uncertainties. We conclude that the WW-approximation works reasonably well, see figure 2 . 
Presently $h_{L}^{a}(x)$ is unknown. With phenomenological information on $h_{1}^{a}(x)$ [63-65], the WW approximation (3.2b) for $h_{L}^{a}(x)$ could be tested experimentally in Drell-Yan [66].

\subsection{Tests in lattice QCD}

The lowest Mellin moments of the PDF $g_{T}^{q}(x)$ were studied in lattice QCD in the quenched approximation [21] and with $N_{f}=2$ flavors of light dynamical quarks [22]. The results obtained were compatible with a small $\tilde{g}_{T}^{q}(x)$. We are not aware of lattice QCD studies related to the PDF $h_{L}^{a}(x)$, and turn now our attention to TMD studies in lattice QCD.

After first exploratory investigations of TMDs on the lattice [67, 68], recent years have witnessed considerable progress and improvements with regard to rigor, realism and methodology. For the latest developments we refer the interested reader to refs. [69-77]. However, numerical results from recent calculations are only available for a subset of observables, and the quantities calculated are not in a form that lends itself to straightforward tests of the WW-type relations as presented in this paper.

For the time being, we content ourselves with rather crude comparisons based on the lattice data published in refs. [67, 68]. These early works explored all nucleon and quark polarizations, but they used a gauge link that does not incorporate the final or initial state interactions present in SIDIS or Drell-Yan experiments. In other words, the transverse momentum dependent quantities computed in $[67,68]$ are not precisely the TMDs measurable in experiment. More caveats will be discussed along the way.

Let us now translate the approximations (3.6a), (3.6b) into expressions for which we have a chance to compare them with available lattice data. For that we multiply the eqs. (3.6a), (3.6b) by $x^{N}$ with $N=0,1,2, \ldots$ and integrate over $x \in[-1,1]$ which yields

$$
\begin{aligned}
& \int_{-1}^{1} d x x^{N} g_{1 T}^{\perp(1) q}(x) \stackrel{\text { WW-type }}{\approx} \frac{1}{N+2} \int_{-1}^{1} d x x^{N+1} g_{1}^{q}(x) \\
& \int_{-1}^{1} d x x^{N} h_{1 L}^{\perp(1) q}(x) \stackrel{\text { WW-type }}{\approx}-\frac{1}{N+3} \int_{-1}^{1} d x x^{N+1} h_{1}^{q}(x) .
\end{aligned}
$$

Here the negative $x$ refer to antiquark distributions $g_{1}^{\bar{q}}(x)=+g_{1}^{q}(-x), h_{1}^{\bar{q}}(x)=-h_{1}^{q}(-x)$, $g_{1 T}^{\perp(1) \bar{q}}(x)=-g_{1 T}^{\perp(1) q}(-x), h_{1 L}^{\perp(1) \bar{q}}(x)=+h_{1 L}^{\perp(1) q}(-x)$ depending on $C$-parity of the involved operators [2]. The right-hand sides of eqs. (3.11), (3.12) are $x$-moments of parton distributions, and those can be obtained from lattice QCD using well-established methods based on operator product expansion. The left-hand sides are moments of TMDs in $x$ and $\boldsymbol{k}_{\perp}$. We have to keep in mind that TMDs diverge for large $\boldsymbol{k}_{\perp}$. Therefore, without regularizing these divergences in a scheme suitable for the comparison of left and right hand side, a test of the above relations is meaningless, even before we get to address the issues of lattice calculations. Let us not give up at this point and take a look at the lattice observables of ref. [68] where TMDs were obtained from amplitudes $\tilde{A}_{i}\left(l^{2}, \ldots\right)$ in Fourier space, where $\boldsymbol{k}_{\perp}$ is encoded in the Fourier conjugate variable $\boldsymbol{\ell}_{\perp}$, which is the transverse displacement of quark operators in the correlator evaluated on the lattice. In Fourier space, the aforementioned divergent behavior for large $\boldsymbol{k}_{\perp}$ translates into strong lattice scale and scheme dependencies at short distances $\boldsymbol{\ell}_{\perp}$ between the quark operators. The $\boldsymbol{k}_{\perp}$ integrals needed 
for the left-hand sides of eqs. (3.11), (3.12) correspond to the amplitudes at $\boldsymbol{\ell}_{\perp}=0$, where scheme and scale dependence is greatest. In ref. [68] Gaussian fits have been performed to the amplitudes excluding data at short quark separations $\boldsymbol{\ell}_{\perp}$. The Gaussians describe the long-range data quite well and bridge the gap at short distances $\ell_{\perp}$. Taking the Gaussian fit at $\boldsymbol{\ell}_{\perp}=0$, we get a value that is (presumably) largely lattice-scheme and scale independent. We have thus swept the problem of divergences under the rug. The Gaussian fit acts as a crude regularization of the divergences that appear in TMDs at large $\boldsymbol{k}_{\perp}$ and manifest themselves as short range artifacts on the lattice. Casting this line of thought into mathematics, we get

$$
\begin{aligned}
& \int_{-1}^{1} d x g_{1 T}^{\perp(1) q}(x)=\int_{-1}^{1} d x \int d^{2} \boldsymbol{k}_{\perp} \frac{k_{\perp}^{2}}{2 M^{2}} g_{1 T}^{\perp q}\left(x, k_{\perp}\right)=-2 \tilde{A}_{7, q}(\ell=0) \stackrel{\text { Gauss }}{=}-c_{7, q} \\
& \int_{-1}^{1} d x h_{1 L}^{\perp(1) q}(x)=\int_{-1}^{1} d x \int d^{2} \boldsymbol{k}_{\perp} \frac{k_{\perp}^{2}}{2 M^{2}} h_{1 T}^{\perp q}\left(x, k_{\perp}\right)=-2 \tilde{A}_{10, q}(\ell=0) \stackrel{\text { Gauss }}{=}-c_{10, q}
\end{aligned}
$$

where the amplitudes $\tilde{A}$ and constants $c$ are those of ref. [68]. We have thus expressed the left-hand side of eqs. (3.11), (3.12) in terms of amplitudes $c_{7, q}$ and $c_{10, q}$ of the Gaussian fits on the lattice. Before quoting numbers, a few more comments are in order. The overall multiplicative renormalization in ref. [68] was fixed by setting the Gaussian integral $c_{2, u-d}$ of the unpolarized TMD $f_{1}$ in the isovector channel ( $\left.\mathrm{u}-\mathrm{d}\right)$ to the nucleon quark content, namely to 1 . One then assumes that the normalization of the lattice results for the unpolarized TMD $f_{1}$ also fixes the normalization for polarized quantities correctly. This assumption holds if renormalization is multiplicative and flavor-independent for the non-local lattice operators. This is not true for all lattice actions [75]. But presumably it is true if the lattice action preserves chiral symmetry, as it does in the present case. The Gaussian fits along with the normalization prescription serve as a crude form of renormalization, and this is needed to attempt a comparison of left and right hand sides of equations eqs. (3.11), (3.12).

There is another issue to discuss. The gauge link that goes into the evaluation of the quark-quark correlator introduces a power divergence that has to be subtracted. Ref. [68] employs a subtraction scheme on the lattice but establishes no connection with a subtraction scheme designed for experimental TMDs and the corresponding gauge-link geometry. The gauge-link renormalization mainly influences the width of the Gaussian fits; the amplitudes are only slightly affected, so it may not play a big role for our discussion. Altogether, the significance of our numerical "tests" of WW relations should be taken with a grain of salt.

For the test of (3.11), we use the numbers $\int d x g_{1 T}^{\perp(1) u}(x) \stackrel{\text { Gauss }}{=}-c_{7, u}=0.1041(85)$ and $\int d x g_{1 T}^{\perp(1) d}(x) \stackrel{\text { Gauss }}{=}-c_{7, d}=-0.0232(42)$ from [68]. Lattice data for $\int d x x^{N} g_{1}^{q}(x)[78,79]$ and $\int d x x^{N} h_{1}^{q}(x)$ [80] are available for $N=0,1,2,3$. These values have been computed using (quasi-)local operators that have been renormalized to the $\overline{M S}$ scheme at the scale $\mu^{2}=4 \mathrm{GeV}^{2}$. According to [79] (data set 4 : with $a m_{u, d}=0.020$ with $m_{\pi} \approx 500 \mathrm{MeV}$ ) one has $\int d x x g_{1}^{u-d}(x)=0.257(10)$ and $\int d x x g_{1}^{u+d}(x)=0.159(14)$. Decomposing the results 
from [79] into individual flavors and inserting them into (3.11), we obtain

$$
\begin{gathered}
\underbrace{\int d x g_{1 T}^{\perp(1) u}(x)}_{=0.1041(85) \text { ref. [68] }} \stackrel{!}{\stackrel{1}{\approx} \int d x x g_{1}^{u}(x)} \\
\underbrace{\int d x g_{1 T}^{\perp(1) d}(x)}_{=-0.104(9) \text { ref. [79] }} \stackrel{!}{\frac{1}{2} \int d x x g_{1}^{d}(x)}
\end{gathered}
$$

which confirms the approximation (3.11) for $N=0$ within the statistical uncertainties of the lattice calculations. In order to test (3.12) we use $\int d x h_{1 L}^{\perp(1) u}(x) \stackrel{\text { Gauss }}{=}-c_{10, u}=$ $-0.0881(72)$ and $\int d x h_{1 L}^{\perp(1) d}(x) \stackrel{\text { Gauss }}{=}-c_{10, d}=0.0137(34)$ from [68] and the lattice data $\int d x x h_{1}^{u}(x)=0.28(1)$ and $\int d x x h_{1}^{d}(x)=-0.054(4)$ from QCDSF [80]. ${ }^{4}$ Inserting these numbers into (3.12) for the case $N=0$ we obtain

$$
\begin{aligned}
&= \underbrace{\int d x h_{1 L}^{\perp(1) u}(x)}_{=-0.0881(72) \text { ref. [68] }} \stackrel{!}{\approx}-\underbrace{-\frac{1}{3} \int d x x h_{1}^{u}(x)}_{=-0.093(3) \text { ref. [80] }}, \\
& \underbrace{\int d x h_{1 L}^{\perp(1) d}(x)}_{=0.0137(34) \text { ref. [68] }} \stackrel{!}{\approx} \underbrace{-\frac{1}{3} \int d x x h_{1}^{d}(x)}_{=0.018(1) \text { ref. [80] }},
\end{aligned}
$$

which again confirms the WW-type approximation within the statistical uncertainties of the lattice calculations.

Several more comments are in order concerning the, at first glance, remarkably good confirmation of the WW-type approximations by lattice data in eqs. (3.15), (3.16).

First, the relations refer to lattice parameters corresponding to pion masses of $500 \mathrm{MeV}$. We do not need to worry about that too much. The lattice results do provide a valid test of the approximations in a "hadronic world" with somewhat heavier pions and nucleons. All that matters in our context is that the relative size of $\bar{q} g q$-matrix elements is small with respect to $\bar{q} q$-matrix elements.

Second, we have to revisit carefully which approximations the above lattice calculations actually test. As mentioned above, in the lattice study [67, 68], a specific choice for the path of the gauge link was chosen, which is actually different from the paths required in SIDIS or Drell-Yan. With the path choice of $[67,68]$ there are effectively only (T-even) $A_{i}$ amplitudes, the $B_{i}$ amplitudes are absent. Therefore the test (3.15) of the WW-type approximation (3.11) actually constitutes a test of the WW-approximation (3.2a) and confirms earlier lattice work [21, 22], (cf. refs. [30, 31] and section 3.6). Similarly, the test (3.16) of the WW-type approximation (3.12) actually constitutes a test of the WWapproximation (3.2b). The latter, however, has not been reported previously in literature, and constitutes a new result.

\footnotetext{
${ }^{4}$ These numbers are read off from a figure in [80], and were computed on a different lattice. We interpolate them to a common value of the pion mass $m_{\pi} \approx 500 \mathrm{MeV}$, and estimate the uncertainty conservatively in order to take systematic effects into account due to the use of a different lattice.
} 
Third, to be precise, (3.15), (3.16) test the first Mellin moments of the WW approximations (3.2a), (3.2b), which corresponds to the Burkhardt-Cottingham sum rule for $g_{T}^{a}(x)$ and an analogous sum rule for $h_{L}^{a}(x)$ (see [55] and references therein). In view of the long debate on the validity of those sum rules [54, 81, 82], this is an interesting result in itself.

It is important to stress that in view of the pioneering and exploratory status of the TMD lattice calculations $[67,68]$, this is already a remarkable and very interesting result. Thus, apart from the instanton calculation [20], also lattice data provide support for the validity of the WW approximation (3.2b). At the same time, however, we also have to admit that we do not really reach our goal of testing the WW-type approximations on the lattice. We have to wait for better lattice data. Meanwhile we may try to gain insights into the quality of WW-type approximations from models.

\subsection{Tests in models}

Effective approaches and models such as bag [17, 83-85], spectator [86], chiral quarksoliton [87], or light-cone constituent [88, 89] models support the approximations (3.2a), (3.2b) for PDFs within an accuracy of $(10-30) \%$ at low hadronic scale below $1 \mathrm{GeV}$.

Turning to TMDs, we recall that in models without gluon degrees of freedom certain relations among TMDs hold, the so-called quark-model Lorentz-invariance relations (qLIRs) $[2,32] .{ }^{5}$ Initially thought to be exact [2,32], qLIRs were shown to be invalid in models with gluons [90, 91] and in QCD [92]. They originate from decomposing the (completely unintegrated) quark correlator in terms of Lorentz-invariant amplitudes, and TMDs are certain integrals over those amplitudes. When gluons are absent, the correlator consists of twelve amplitudes [2, 32], i.e., fewer amplitudes than TMDs, which implies relations: the qLIRs. In QCD, the correct Lorentz decomposition requires the consideration of gauge links, which introduces further amplitudes. As a result one has as many amplitudes as TMDs and no relations exist [92]. However, qLIRs "hold" in QCD in the WW-type approximation [30]. In models without gluon degrees of freedom they are exact $[30,31,85,86]$.

The bag, spectator, and light-cone constituent-quark models support the approximations (3.6a), (3.6b) within an accuracy of (10-30)\% [85, 86, 88, 89]. The spectator and bag model support WW-type approximations within (10-30)\% [85]. As they are defined in terms of quark bilinear expressions (2.10), it is possible to evaluate twist-3 functions in quark models [17]. The tilde-terms arise due to the different model interactions, and it is important to discuss critically how realistically they describe the $\bar{q} g q$-terms of QCD [93, 94].

In the covariant parton model with intrinsic 3D-symmetric parton orbital motion [95], quarks are free, $\bar{q} g q$ correlations absent, and all WW and WW-type relations exact [96, 97]. The phenomenological success of this approach [95] may hint at a general smallness of $\bar{q} g q$ terms, although some of the predictions from this model have yet to be tested [96].

Noteworthy is the result from the chiral quark-soliton model where the WW-type approximation (3.3b) happens to be exact: $x f^{\perp q}\left(x, k_{\perp}^{2}\right)=f_{1}^{q}\left(x, k_{\perp}^{2}\right)$ for quarks and anti-

\footnotetext{
${ }^{5}$ Notice that the qLIRs of $[2,32]$ are valid only in quark models with no gluons and should not be confused with the LIRs of [56], which are exact relations in QCD, see section 3.1. In the literature, both are often simply referred to as LIRs. This ambiguity is unfortunate.
} 
quarks [93]. The degrees of freedom in this model are quarks, antiquarks, and Goldstone bosons, which are strongly coupled (the coupling constant is $\sim 4$ ) and has to be solved using nonperturbative techniques (expansion in $1 / N_{c}$, where $N_{c}$ is the number of colors) with the nucleon described as a chiral soliton. In general, the model predicts non-zero tilde-terms, for instance $\tilde{e}^{a}(x) \neq 0$ [98-100]. However, despite strong interactions in this effective theory, the tilde term $\tilde{f}^{\perp q}\left(x, k_{\perp}^{2}\right)$ vanishes exactly in this model [93] and the WW-type approximation (3.3b) becomes exact at the low initial scale of this model of $\mu_{0} \sim 0.6 \mathrm{GeV}$.

Let us finally discuss quark-target models, where gluon degrees of freedom are included and WW(-type) approximations badly violated [90, 91, 101, 102]. This is natural in this class of models for two reasons. First, quark-mass terms are of $\mathcal{O}\left(m_{q} / M_{N}\right)$ and negligible in the nucleon case, but of $\mathcal{O}(100 \%)$ in a quark target where $m_{q}$ plays also the role of $M_{N}$. Second, even if one refrains from mass terms the approximations are spoiled by gluon radiation, see for instance [103] in the context of (3.2a). This means that perturbative QCD does not support the WW-approximations: they certainly are not preserved by evolution. However, scaling violations per se do not need to be large. What is crucial in this context are dynamical reasons for the smallness of the matrix elements of $\bar{q} g q$-operators. This requires the consideration of chiral symmetry breaking effects reflected in the hadronic spectrum, as considered in the instanton vacuum model $[19,20]$ but out of scope in quarktarget models.

We are not aware of systematic tests of WW-type approximations for FFs. One information worth mentioning in this context is that in spectator models [86] tilde-contributions to FFs are proportional to the offshellness of partons $[93,94]$. This natural feature may indicate that in the region dominated by effects of small $P_{\perp}$ tilde-terms might be small. On the other hand, quarks have sizable constituent masses of the order of few hundred $\mathrm{MeV}$ in spectator models and the mass-terms are not small. The applicability of WW-type approximations to FFs remains the least tested point in our approach.

\subsection{Basis functions for the WW-type approximations}

The 6 leading-twist TMDs $f_{1}^{a}, f_{1 T}^{\perp a}, g_{1}^{a}, h_{1}^{a}, h_{1}^{\perp a}, h_{1 T}^{\perp a}$ and 2 leading-twist FFs $D_{1}^{a}, H_{1}^{\perp a}$ provide a basis in the sense that in WW-type approximation all other TMDs and FFs can either be expressed in terms of these basis functions or vanish. Below we shall see that, under the assumption of the validity of WW-type approximations, it is possible to express all SIDIS structure functions in terms of the basis functions. ${ }^{6}$ These basis functions allow us to describe, in WW-type approximation, all other TMDs. The experiment will tell us how well the approximations work. In some cases, however, we know in advance that the WW-type approximations have limitations, see next section 3.8.

\footnotetext{
${ }^{6}$ Notice that SIDIS alone is not sufficient to uniquely determine the eight basis functions that appear in six SIDIS leading-twist structure functions. It is thus crucial to take advantage of other processes (like Drell-Yan and hadron production in $e^{+} e^{-}$annihilation, which are indispensable for the determination of $\left.f_{1}^{a}, D_{1}^{a}, H_{1}^{\perp a}\right)$.
} 


\subsection{Limitation of WW-type approximations}

The approximation may work in the case when a TMD or $\mathrm{FF}=\langle\bar{q} q\rangle+\langle\bar{q} g q\rangle \approx\langle\bar{q} q\rangle \neq 0$ with a "controlled approximation" in the spirit of eq. (3.1). We know cases where this works, see sections 3.3, 3.4, but it has to be checked case by case whether $|\langle\bar{q} g q\rangle| \ll|\langle\bar{q} q\rangle|$ for a given operator. At least in such cases the approximation has a chance to work.

However, it may happen that after applying the QCD equations of motion one ends up in the situation that a given function $=\langle\bar{q} q\rangle+\langle\bar{q} g q\rangle$ with $\langle\bar{q} q\rangle=0$. This happens for the T-even TMD $e^{a}$ in eqs. (3.2c), (3.3a), for the T-odd TMDs $e_{L}^{q}, e_{T}^{q}, e_{T}^{\perp q}, f_{L}^{\perp q}, g^{\perp q}$ in eqs. (3.4a)-(3.4e), and for the FFs $E^{q}, G^{\perp q}$ in eqs. (3.7a), (3.7b) (actually, all twist-3 FFs are affected, we will discuss this in detail below). In this situation the "leading term" is absent, so neglecting the "subleading (pure twist-3) term" actually constitutes an error of $100 \%$ even if the neglected matrix element $\langle\bar{q} g q\rangle$ is very small. Notice that this occurs for all subleading-twist FFs that enter SIDIS structure functions only in the shape of tilde-FFs, see section 2 and eqs. (2.18). We shall see that some structure functions are potentially more and others potentially less affected by this generic limitation. In any case, phenomenological work has to be carried out to find out whether or not the approximation works.

For both FFs and TMDs there are also limitations which go beyond this generic issue. To illustrate this for FFs we recall that both $H_{1}^{\perp(1) q}$ and $\tilde{H}_{1}^{q}$ are related to integrals of an underlying function $H_{F U}^{q, \Im}\left(z, z_{1}\right)$ as pointed out in ref. [56]. Therefore, if one literally assumed $\tilde{H}^{q}(z)$ to be zero, this would imply that also $H_{1}^{\perp(1) q}$ would vanish, indicating that the WW-type approximation has to be used with care for chiral-odd FFs.

Similar limitations exist also for TMDs. This is manifest in particular for those twist-3 T-odd TMDs that appear in the decomposition of the correlator (2.10) with no prefactor of $k_{\perp}$. There are three cases: $f_{T}^{a}\left(x, k_{\perp}^{2}\right), h^{a}\left(x, k_{\perp}^{2}\right)$, and $e_{L}^{a}\left(x, k_{\perp}^{2}\right)$. Such TMDs in principle survive integration of the correlator over $k_{\perp}$ and would have PDF counterparts if there were not the sum rules in eq. (2.13). These sum rules arise because hypothetical PDF versions of T-odd TMDs vanish: they have a simple straight gauge link along the lightcone, and such objects vanish due to parity and time-reversal symmetry of strong interactions. This argument does not apply to other T-odd TMDs because they drop out from the $k_{\perp^{-}}$ integrated correlator due to explicit factors of, e.g., $k_{\perp}^{j}$ in the case of the Sivers function.

Let us first discuss the case of $f_{T}^{a}\left(x, k_{\perp}^{2}\right)$. Taking the WW-type approximation $(3.4 \mathrm{~g})$ literally means $x \int d^{2} k_{\perp} f_{T}^{a}\left(x, k_{\perp}^{2}\right) \stackrel{! ?}{=}-f_{1 T}^{\perp(1) a}(x) \neq 0$, at variance with the sum rule (2.13). We have $x f_{T}^{a}\left(x, k_{\perp}\right)=x \tilde{f}_{T}^{a}\left(x, k_{\perp}^{2}\right)-f_{1 T}^{\perp(1) a}\left(x, k_{\perp}^{2}\right)$ from QCD equations of motion [5], which yields $(3.4 \mathrm{~g})$. The point is that in this case it is essential to keep the tilde-function. The situation for the chirally and T-odd twist-3 TMD $h^{a}\left(x, k_{\perp}^{2}\right)$ is analogous. The third function in (2.13) causes no issues since $e_{L}^{a}\left(x, k_{\perp}^{2}\right)=\tilde{e}_{L}^{a}\left(x, k_{\perp}^{2}\right) \approx 0$ in WW-type approximation.

Does it mean WW-type approximations fail for $f_{T}^{a}\left(x, k_{\perp}^{2}\right)$ and $h^{a}\left(x, k_{\perp}^{2}\right)$ ? Not necessarily! The approximations may work in some but not all regions of $k_{\perp}$, but the sum rules (2.13) include integration over all $k_{\perp}$. Notice also that, e.g., $f_{1 T}^{\perp(1), q}(x)$ is related to the soft-gluon-pole matrix element $T_{F}(x, x)[104,105]$, which is a $\bar{q} g q$-term that one would naturally neglect in WW-type approximation. In this sense $(3.4 \mathrm{~g})$ could be consistent. Thus, issues with the sum rules (2.13) do not need to exclude the possibility that the WW- 
type approximations for $f_{T}^{a}\left(x, k_{\perp}^{2}\right)$ and $h^{a}\left(x, k_{\perp}^{2}\right)$ in $(3.4 \mathrm{~g}),(3.4 \mathrm{~h})$ may work at small $k_{\perp}$ where we use them in our TMD approach. This would mean that the UV region is essential to realize the sum rules (2.13). Alternatively, one could also envision the sum rules (2.13) to be sensitive to the IR region through gluonic or fermionic pole contributions manifest in tilde-terms. Presently too little is known in the theory of subleading-twist TMDs. In sections 7.6 and 7.8 we will present pragmatic solutions for how to deal with the TMDs $f_{T}^{a}\left(x, k_{\perp}^{2}\right)$ and $h^{a}\left(x, k_{\perp}^{2}\right)$ phenomenologically. For now let us keep in mind that one has to keep a vigilant eye on all WW-type approximations, and especially on those for $f_{T}^{a}\left(x, k_{\perp}^{2}\right)$ and $h^{a}\left(x, k_{\perp}^{2}\right)$.

As it was mentioned in the Introduction one important limitation concerns the fact that the WW-type approximations are not preserved under $Q^{2}$ evolution. Still some intuition can be obtained from the collinear case: the evolution equations for $g_{T}^{a}(x)$ and $h_{L}^{a}(x)$ exhibit complicated mixing patterns typical for higher twist functions, which simplify to DGLAP-type evolutions in the limit of a large number of colors $N_{c}$ and in the limit of large- $x$ [106-109]. These evolution equations differ from those of the leading-twist functions $g_{1}^{a}(x)$ and $h_{1}^{a}(x)$. However, since $Q^{2}$ varies moderately in the considered experiments (e.g. for common values of $x$ the $Q^{2}$ at COMPASS is only about a factor 2-3 larger than at HERMES), this point is not a major uncertainty in our study. More theoretical work will be required to understand $k_{\perp}$-evolution effects of subleading twist TMDs in future experiments (EIC) covering kinematic regions that vary by orders of magnitude in $Q^{2}$.

\section{SIDIS in the WW-type approximation and Gaussian model}

In this section, we consequently apply the WW and WW-type approximation to SIDIS, and describe our procedure to evaluate the structure functions in this approximation and the Gaussian Ansatz which we use to model the $k_{\perp}$ dependence of TMDs.

\subsection{Leading structure functions amenable to WW-type approximations}

The WW and WW-type approximations are useful for the following two leading-twist structure functions:

$$
\begin{gathered}
F_{L T}^{\cos \left(\phi_{h}-\phi_{S}\right)} \stackrel{\mathrm{WW}}{=} \mathcal{C}\left[\omega_{\mathrm{B}}^{\{1\}} g_{1 T}^{\perp} D_{1}\right] \mid \begin{array}{l}
g_{1 T}^{\perp a} \rightarrow g_{1}^{a} \\
\text { eq. (3.6a) }
\end{array} \\
\left.F_{U L}^{\sin 2 \phi_{h}} \stackrel{\mathrm{WW}}{=} \mathcal{C}\left[\omega_{\mathrm{AB}}^{\{2\}} h_{1 L}^{\perp} H_{1}^{\perp}\right]\right|_{\substack{h_{1 L}^{\perp a} \rightarrow h_{1}^{a} \\
\text { eq. }(3.6 \mathrm{~b})}} .
\end{gathered}
$$




\subsection{Subleading structure functions in WW-type approximations}

In the case of the subleading-twist structure functions the WW-type approximations in (3.3a)-(3.4h) lead to considerable simplifications. We obtain the approximations

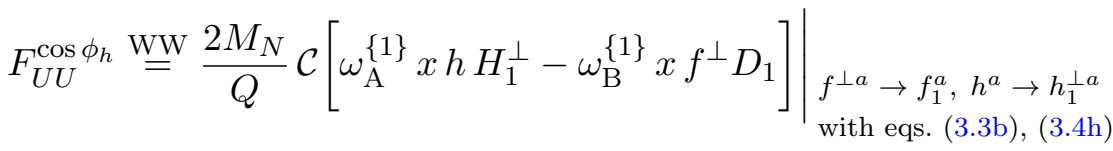

$$
\begin{aligned}
& \left.F_{U L}^{\sin \phi_{h}} \stackrel{\mathrm{wW}}{=} \frac{2 M_{N}}{Q} \mathcal{C}\left[\omega_{\mathrm{A}}^{\{1\}} x h_{L} H_{1}^{\perp}\right]\right|_{\substack{h_{L}^{a} \rightarrow h_{1 L}^{\perp a} \\
(3.3 \mathrm{f})}} \\
& F_{U T}^{\sin \phi_{S}} \stackrel{\mathrm{WW}}{=} \frac{2 M_{N}}{Q} \mathcal{C}\left[\omega^{\{0\}} x f_{T} D_{1}-\frac{\omega_{\mathrm{B}}^{\{2\}}}{2}\left(x h_{T}-x h_{T}^{\perp}\right) H_{1}^{\perp}\right] \mid \begin{array}{l}
f_{T}^{a} \rightarrow f_{1 T}^{\perp a}, \\
h_{T}^{a}-h_{T}^{\perp a} \rightarrow h_{1}^{a} \\
(3.4 \mathrm{~g}),(3.3 \mathrm{~g}),(3.3 \mathrm{~h})
\end{array} \\
& F_{U T}^{\sin \left(2 \phi_{h}-\phi_{S}\right)} \stackrel{\mathrm{wW}}{=} \frac{2 M_{N}}{Q} \mathcal{C}\left[\omega_{\mathrm{C}}^{\{2\}} x f_{T}^{\perp} D_{1}+\frac{\omega_{\mathrm{AB}}^{\{2\}}}{2} x\left(h_{T}+h_{T}^{\perp}\right) H_{1}^{\perp}\right] \mid \begin{array}{l}
f_{T}^{\perp a} \rightarrow f_{1 T}^{\perp a}, \\
\left(h_{T}^{a}+h_{T}^{\perp a}\right) \rightarrow h_{1 T}^{\perp a} \\
(3.4 \mathrm{f}),(3.3 \mathrm{~g}),(3.3 \mathrm{~h})
\end{array} \\
& F_{L U}^{\sin \phi_{h}} \stackrel{\mathrm{WW}}{=} 0 \\
& \left.F_{L T}^{\cos \phi_{S}} \stackrel{\mathrm{Ww}}{=} \frac{2 M_{N}}{Q} \mathcal{C}\left[-\omega^{\{0\}} x g_{T} D_{1}\right]\right|_{\substack{g_{T}^{a} \rightarrow g_{1}^{a} \\
(3.2 \mathrm{a})}} \\
& \left.F_{L L}^{\cos \phi_{h}} \stackrel{\mathrm{WW}}{=} \frac{2 M_{N}}{Q} \mathcal{C}\left[-\omega_{\mathrm{B}}^{\{1\}} x g_{L}^{\perp} D_{1}\right]\right|_{\substack{g_{L}^{\perp a} \rightarrow g_{1}^{a} \\
(3.3 \mathrm{c})}} \\
& F_{L T}^{\cos \left(2 \phi_{h}-\phi_{S}\right)} \stackrel{\mathrm{WW}}{=} \frac{2 M_{N}}{Q} \mathcal{C}\left[-\omega_{\mathrm{C}}^{\{2\}} x g_{T}^{\perp} D_{1}\right] \mid \begin{array}{l}
g_{T}^{\perp} a \rightarrow g_{1}^{a} \\
(3.3 \mathrm{~d}),(3.6 \mathrm{a})
\end{array}
\end{aligned}
$$

\subsection{Gaussian Ansatz for TMDs and FFs}

In this work we will use the so-called Gaussian Ansatz for the TMDs and FFs. This Ansatz, which for a generic TMD or FF is given by

$$
f\left(x, k_{\perp}^{2}\right)=f(x) \frac{e^{-k_{\perp}^{2} /\left\langle k_{\perp}^{2}\right\rangle}}{\pi\left\langle k_{\perp}^{2}\right\rangle}, \quad D\left(z, P_{\perp}^{2}\right)=D(z) \frac{e^{-P_{\perp}^{2} /\left\langle P_{\perp}^{2}\right\rangle}}{\pi\left\langle P_{\perp}^{2}\right\rangle},
$$

is popular not only because it considerably simplifies the calculations. In fact, all convolution integrals of the type (2.16) can be solved analytically with this Ansatz. Far more important is the fact that it works phenomenologically with a good accuracy in many practical applications [110-115]. Of course this Ansatz is only a rough approximation. For instance, it is not consistent with general matching expectations for large $k_{\perp}$ [116].

Nevertheless, if one limits oneself to work in a regime where the transverse momenta (of hadrons produced in SIDIS, dileptons produced in the Drell-Yan process, etc.) are small 
compared to the hard scale in the process, then the Ansatz works quantitatively very well. The most recent and detailed tests were reported in [113], where the Gaussian Ansatz was shown to describe the most recent SIDIS data: no deviations were observed within the error bars of the data provided one takes into account the broadening of the Gaussian widths with increasing energy [113] according with expectations from QCD [43]. The Gaussian Ansatz is approximately compatible with the $k_{\perp}$-shapes obtained from evolution [43] or fits to highenergy Tevatron data on weak-boson production [117]. Effective models at low [85, 88, 89] and high [97] renormalization scales support this Ansatz as a good approximation.

\subsection{Evaluation of structure functions in WW-type \& Gaussian approximation}

The Gaussian Ansatz is compatible with many WW-type approximations, but not all. The trivial approximations (3.3a) and (3.4a)-(3.4e) cause no issue. The Gaussian Ansatz can also be applied to the nontrivial approximations in eqs. (3.3b)-(3.3d) and (3.4f), provided the corresponding Gaussian widths are defined to be equal to each other: for example, in the WW-type approximation $(3.3 \mathrm{~b}), x f^{\perp q}\left(x, k_{\perp}^{2}\right) \approx f_{1}^{q}\left(x, k_{\perp}^{2}\right)$, one may assume Gaussian $k_{\perp}$-dependence for $f^{\perp q}\left(x, k_{\perp}^{2}\right)$ and for $f_{1}^{q}\left(x, k_{\perp}^{2}\right)$ as long as the Gaussian widths of these two TMDs are assumed to be equal.

In the case of the approximations $(3.3 \mathrm{e})-(3.3 \mathrm{~h})$ the situation is different because here twist-3 TMDs are related to transverse moments of twist-2 TMDs. In such cases the Gaussian Ansatz is not compatible with the WW-type approximations: for instance, the approximation (3.3e) relates $x g_{T}^{q}\left(x, k_{\perp}^{2}\right) \approx \frac{k_{\perp}^{2}}{2 M_{N}^{2}} g_{1 T}^{q}\left(x, k_{\perp}^{2}\right)$, e.g., if $g_{1 T}^{q}\left(x, k_{\perp}^{2}\right)$ was exactly Gaussian then $g_{T}^{q}\left(x, k_{\perp}^{2}\right)$ certainly could not be Gaussian. If one wanted to take the Gaussian Ansatz and WW-type approximations literally, one clearly would deal with an incompatibility. However, we of course must keep in mind that both are approximations.

Some comments are in order to understand how the usage of the Gaussian Ansatz and the WW-type approximations can be reconciled. First, let us remark that the individual TMDs, say $g_{T}^{q}\left(x, k_{\perp}^{2}\right)$ and $g_{1 T}^{q}\left(x, k_{\perp}^{2}\right)$ in our example, may each by itself be assumed to be approximately Gaussian in $k_{\perp}$, which is supported by quark model calculations [85]. Second, we actually do not need the unintegrated WW-type approximations. For phenomenological applications we can use the WW-type approximations in "integrated form."

Let us stress that if one took an unintegrated WW-type approximation of the type $x g_{T}^{q}\left(x, k_{\perp}^{2}\right) \approx \frac{k_{\perp}^{2}}{2 M_{N}^{2}} g_{1 T}^{q}\left(x, k_{\perp}^{2}\right)$ literally and assumed both TMDs to be exactly Gaussian, one would find "incompatibilities", perhaps most strikingly in the limit $k_{\perp} \rightarrow 0$ where the left-hand side is finite while the right-hand side vanishes. Notice that the failure of the WW-type approximations (3.3e)-(3.3h) in the limit $k_{\perp} \rightarrow 0$ is not specific to the Gaussian model, but a general feature caused by neglecting tilde-terms. This indicates a practical scheme how to use responsibly the WW-type approximations in eqs. (3.3e)-(3.3h).

Our procedure is as follows. In a first step we assume that all TMDs and FFs are (approximately) Gaussian and solve the convolution integrals. In the second step we use the integrated WW-type approximations to simplify the results for the structure functions.

Notice that in some cases (when T-even TMDs are involved) one could choose a different order of the steps: first apply WW-type approximations and then solve convolution 

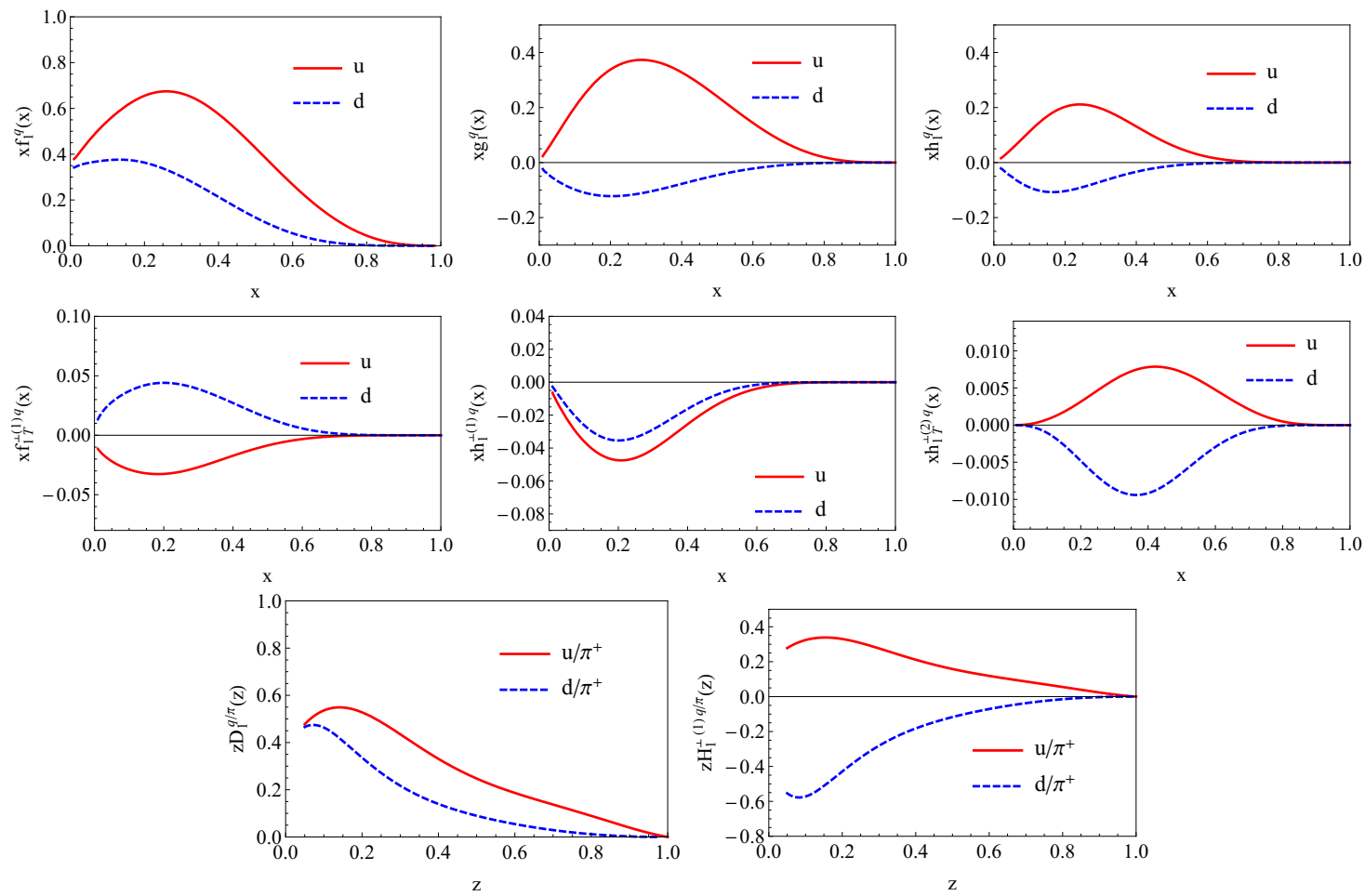

Figure 3. The basis functions $f_{1}^{a}, g_{1}^{a}, h_{1}^{a}, f_{1 T}^{\perp a}, h_{1}^{\perp a}, h_{1 T}^{\perp a} ; D_{1}^{a}, H_{1}^{\perp a}$. The parametrizations of the basis functions and the Gaussian model parameters are described in detail in appendix A.

integrals with Gaussian Ansatz. In general, this would yield different (and bulkier) analytical expressions, but we convinced ourselves that the differences are numerically within the accuracy expected for this approach. However, for the structure functions discussed in sections 7.6 and 7.8, such an "alternative scheme" would give results at variance with the sum rules for the twist-3 T-odd TMDs in eq. (2.13), as discussed in section 3.8. The scheme presented here will allow us to implement those sum rules in a convenient and consistent way. We will follow up on this in more detail in sections 7.6 and 7.8.

To summarize, our procedure is to solve first the convolution integrals with a Gaussian Ansatz, and use then WW-type approximations. When implementing this procedure we will see that the results for the structure functions can be conveniently expressed in terms of the basis TMDs or their adequate transverse moments.

\subsection{Phenomenological information on basis functions}

We have seen that the following 6 TMDs and 2 FFs provide a basis (section 3) and allow us to express all SIDIS structure functions (section 4) in WW-type approximation:

$$
\text { basis: } f_{1}^{a}, f_{1 T}^{\perp a}, g_{1}^{a}, h_{1}^{a}, h_{1}^{\perp a}, h_{1 T}^{\perp a} ; D_{1}^{a}, H_{1}^{\perp a} .
$$

Phenomenological information is available for all basis functions at least to some extent. In figure 3 we present plots of the basis functions, and refer to appendix A for details. The four functions $f_{1}^{a}, g_{1}^{a}, h_{1}^{a}, D_{1}^{a}$ are related to twist-2 collinear functions. All collinear functions are calculated at $Q^{2}=2.4 \mathrm{GeV}^{2}$ with $f_{1}^{a}(x)$ from [118], $g_{1}^{a}(x)$ from [62], and $D_{1}^{a}(z)$ 
from [119]. The other four TMDs have no collinear counterparts. For $f_{1 T}^{\perp a}, h_{1}^{\perp a}$, and $H_{1}^{\perp a}$ it is convenient to consider their (1)-moments, for $h_{1 T}^{\perp a}$ the (2)-moment; see (B.8) for definitions. This has two important advantages. First, this step simplifies the Gaussian model expressions, and the Gaussian width parameters are largely absorbed in the definitions of the transverse moments. Second, the $k_{\perp}$-moments of these TMDs have in principle simple definitions in QCD (whereas, e.g., the function $f_{1 T}^{\perp a}(x)$ can be computed in models but is very cumbersome to define in $\mathrm{QCD}$ ). The parametrizations for the basis functions read

$$
\begin{aligned}
f_{1}^{a}\left(x, k_{\perp}^{2}\right) & =f_{1}^{a}(x) \frac{1}{\pi\left\langle k_{\perp}^{2}\right\rangle_{f_{1}}} e^{-k_{\perp}^{2} /\left\langle k_{\perp}^{2}\right\rangle_{f_{1}}}, \\
D_{1}^{a}\left(z, P_{\perp}^{2}\right) & =D_{1}^{a}(z) \frac{1}{\pi\left\langle P_{\perp}^{2}\right\rangle_{D_{1}}} e^{-P_{\perp}^{2} /\left\langle P_{\perp}^{2}\right\rangle_{D_{1}}}, \\
g_{1}^{a}\left(x, k_{\perp}^{2}\right) & =g_{1}^{a}(x) \frac{1}{\pi\left\langle k_{\perp}^{2}\right\rangle_{g_{1}}} e^{-k_{\perp}^{2} /\left\langle k_{\perp}^{2}\right\rangle_{g_{1}}}, \\
h_{1}^{a}\left(x, k_{\perp}^{2}\right) & =h_{1}^{a}(x) \frac{1}{\pi\left\langle k_{\perp}^{2}\right\rangle_{h_{1}}} e^{-k_{\perp}^{2} /\left\langle k_{\perp}^{2}\right\rangle_{h_{1}}}, \\
H_{1}^{\perp a}\left(z, P_{\perp}^{2}\right) & =H_{1}^{\perp(1) a}(z) \frac{2 z^{2} m_{h}^{2}}{\pi\left\langle P_{\perp}^{2}\right\rangle_{H_{1}^{\perp}}^{2}} e^{-P_{\perp}^{2} /\left\langle P_{\perp}^{2}\right\rangle_{H_{1}^{\perp}}}, \\
f_{1 T}^{\perp a}\left(x, k_{\perp}^{2}\right) & =f_{1 T}^{\perp(1) a}(x) \frac{2 M^{2}}{\pi\left\langle k_{\perp}^{2}\right\rangle_{f_{1 T}^{\perp}}^{2}} e^{-k_{\perp}^{2} /\left\langle k_{\perp}^{2}\right\rangle_{f_{1 T}^{\perp}}}, \\
h_{1}^{\perp a}\left(x, k_{\perp}^{2}\right) & =h_{1}^{\perp(1) a}(x) \frac{2 M^{2}}{\pi\left\langle k_{\perp}^{2}\right\rangle_{h_{1}^{\perp}}^{2}} e^{-k_{\perp}^{2} /\left\langle k_{\perp}^{2}\right\rangle_{h_{1}^{\perp}}}, \\
h_{1 T}^{\perp a}\left(x, k_{\perp}^{2}\right) & =h_{1 T}^{\perp(2) a}(x) \frac{2 M^{4}}{\pi\left\langle k_{\perp}^{2}\right\rangle_{h_{1 T}^{\perp}}^{3}} e^{-k_{\perp}^{2} /\left\langle k_{\perp}^{2}\right\rangle_{h_{1 T}}} .
\end{aligned}
$$

\section{$5 \quad$ Leading-twist asymmetries and basis functions}

In this section we review how the basis functions describe available SIDIS data. This is of importance to assess the reliability of the predictions presented in the next sections.

\subsection{Leading-twist $\boldsymbol{F}_{U U}$ and Gaussian Ansatz}

As explained in section 4.3 the Gaussian Ansatz is chosen not only because it considerably simplifies the calculations, but more importantly because it works phenomenologically with a good accuracy in many processes including SIDIS [110-115].

The Gaussian Ansatz for the unpolarized TMD and FF is given by eqs. (4.5a), (4.5b). The parameters $\left\langle k_{\perp}^{2}\right\rangle_{f_{1}}$ and $\left\langle P_{\perp}^{2}\right\rangle_{D_{1}}$ can be assumed to be flavor- and $x$ - or $z$-independent, as present data hardly allow us to constrain too many parameters, see appendix A.1 for a review. This assumption can be relaxed, e.g., theoretical studies in chiral effective theories predict a strong flavor-dependence in the $k_{\perp}$-behavior of sea and valence quark TMDs [120]. 

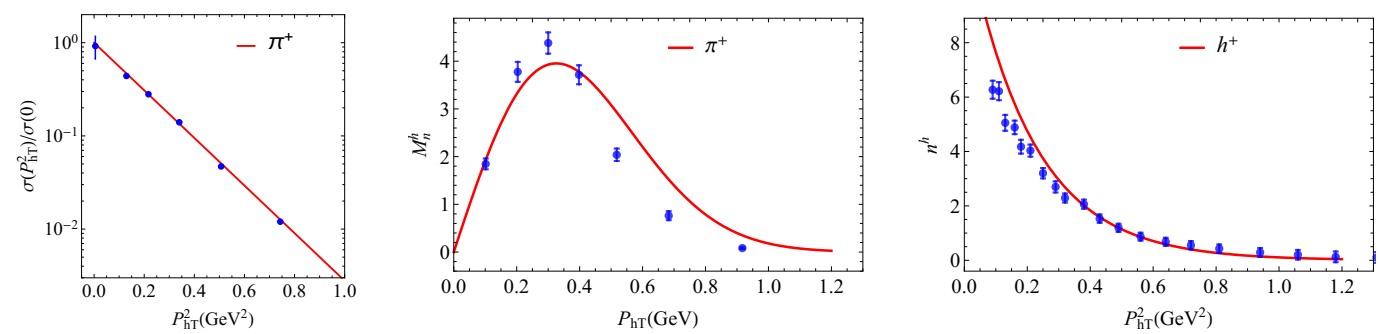

Figure 4. Left panel: $F_{U U}\left(P_{h T}^{2}\right) / F_{U U}(0)$ for $\pi^{+}$production at JLab with a $5.75 \mathrm{GeV} e^{-}$beam [122]. Middle panel: HERMES multiplicity (5.3) at $\left\langle Q^{2}\right\rangle=2.87 \mathrm{GeV}^{2},\langle x\rangle=0.15,\langle z\rangle=0.22$ [123]. Right panel: COMPASS multiplicity (5.4) at $\left\langle Q^{2}\right\rangle=20 \mathrm{GeV}^{2},\langle x\rangle=0.15,\langle z\rangle=0.2$ [124].

The structure function $F_{U U}$ needed for our analysis reads

$$
\begin{aligned}
F_{U U}\left(x, z, P_{h T}\right) & =x \sum_{q} e_{q}^{2} f_{1}^{q}(x) D_{1}^{q}(z) \mathcal{G}\left(P_{h T}\right), \\
F_{U U}(x, z) & =x \sum_{q} e_{q}^{2} f_{1}^{q}(x) D_{1}^{q}(z)
\end{aligned}
$$

where we introduce the notation $\mathcal{G}\left(P_{h T}\right)$, which is defined as

$$
\mathcal{G}\left(P_{h T}\right)=\frac{\exp \left(-P_{h T}^{2} / \lambda\right)}{\pi \lambda}, \quad \lambda=z^{2}\left\langle k_{\perp}^{2}\right\rangle_{f_{1}}+\left\langle P_{\perp}^{2}\right\rangle_{D_{1}}
$$

with the understanding that the convenient abbreviation $\lambda$ is expressed in terms of the Gaussian widths of the preceding TMD and FF. Notice that $\mathcal{G}\left(P_{h T}\right) \equiv \mathcal{G}\left(x, z, P_{h T}\right)$ and that in general $\mathcal{G}\left(P_{h T}\right)$ appears under the flavor sum due to a possible flavor-dependence of the involved Gaussian widths. The normalization $\int d^{2} P_{h T} \mathcal{G}\left(P_{h T}\right)=1$ correctly connects the structure function $F_{U U}\left(x, z, P_{h T}\right)$ in (5.1a) with its $P_{h T}$-integrated counterpart (5.1b). In our effective description this step is trivial. In QCD the connection of TMDs to PDFs is subtle [121]. Figure 4 illustrates how the Gaussian Ansatz describes selected SIDIS data.

Let us begin with JLab where, in the pre- $12 \mathrm{GeV}$ era, electron beams from CEBAF with energies in the range 4.3 to $5.7 \mathrm{GeV}$ were scattered off proton or deuterium targets in the typical kinematics $1 \mathrm{GeV}^{2}<Q^{2}<4.5 \mathrm{GeV}^{2}, W>2 \mathrm{GeV}, 0.1<x<0.6, y<0.85$, $0.5<z<0.8$. The left panel of figure 4 shows basically the SIDIS structure function $F_{U U}\left(P_{h T}^{2}\right)$ normalized with respect to its value at zero transverse hadron momentum ${ }^{7}$ for $\pi^{+}$production from a proton target measured in the CLAS experiment with a $5.75 \mathrm{GeV}$ beam for the kinematics $\left\langle Q^{2}\right\rangle=2.37 \mathrm{GeV}^{2},\langle x\rangle=0.24,\langle z\rangle=0.30$ [122]. Clearly, the Gaussian model works for the entire region of $P_{h T}$ covered in this experiment, in which the structure function $F_{U U}$ falls down by 2 orders of magnitude [113].

Next we discuss a representative plot from the HERMES experiment where pions or kaons were measured in the scattering of $27.6 \mathrm{GeV}$ electrons or positrons of HERA's polarized lepton storage ring off proton and deuteron targets in the SIDIS kinematics

\footnotetext{
${ }^{7}$ Strictly speaking in [122] data for the normalized SIDIS cross section was presented. But these data correspond to $F_{U U}\left(P_{h T}^{2}\right) / F_{U U}(0) \equiv F_{U U}\left(\langle x\rangle,\langle z\rangle, P_{h T}^{2}\right) / F_{U U}(\langle x\rangle,\langle z\rangle, 0)$ up to $1 / Q^{2}$-suppressed terms.
} 
$Q^{2}>1 \mathrm{GeV}^{2}, W^{2}>10 \mathrm{GeV}^{2}, 0.023<x<0.4, y<0.85,0.2<z<0.7$. The middle panel of figure 4 displays the HERMES multiplicity [123]

$$
M_{n}^{h}\left(x, z, P_{h T}\right) \equiv \frac{d \sigma_{\mathrm{SIDIS}}\left(x, z, P_{h T}\right) / d x d z d P_{h T}}{d \sigma_{\mathrm{DIS}}(x) / d x}=2 \pi P_{h T} \frac{F_{U U}\left(x, z, P_{h T}\right)}{x \sum_{q} e_{q}^{2} f_{1}^{q}(x)}
$$

at $\left\langle Q^{2}\right\rangle=2.87 \mathrm{GeV}^{2},\langle x\rangle=0.15,\langle z\rangle=0.22$ for $\pi^{+}$production on the proton target [123].

Finally we show also a representative plot from the COMPASS experiment where charged pions, kaons, or hadrons were measured with $160 \mathrm{GeV}$ longitudinally polarized muons scattered off proton and deuteron targets in the typical SIDIS kinematics $Q^{2}>1 \mathrm{GeV}^{2}, W>5 \mathrm{GeV}, 0.003<x<0.7,0.1<y<0.9,0.2<z<1$. The right panel of figure 4 shows the COMPASS multiplicity [124]

$$
n^{h}\left(x, z, P_{h T}^{2}\right) \equiv \frac{d \sigma_{\mathrm{SIDIS}}\left(x, z, P_{h T}^{2}\right) / d x d z d P_{h T}^{2}}{d \sigma_{\mathrm{DIS}}(x) / d x}=\pi \frac{F_{U U}\left(x, z, P_{h T}^{2}\right)}{x \sum_{q} e_{q}^{2} f_{1}^{q}(x)}
$$

at $\left\langle Q^{2}\right\rangle=20 \mathrm{GeV}^{2},\langle x\rangle=0.15,\langle z\rangle=0.2$ for $h^{+}$production on the deuterium target [124].

To streamline the presentation we refer to the comprehensive appendix $\mathrm{A}$ on the parametrizations used, and for technical details on the Gaussian Ansatz to appendix B.

The description of the HERMES and COMPASS multiplicities in figure 4 is good and sufficient for our purposes, but it is not perfect. The descriptions of the COMPASS data in the region of small $P_{h T}^{2}$ and that of the HERMES data for $P_{h T} \gtrsim 0.3 \mathrm{GeV}$ are not ideal. However, notice that in our description we use the Gaussian widths as fitted and employed in the original extractions of the TMDs. These values were not optimized to fit the HERMES or COMPASS multiplicities. Keeping this in mind, the description in figure 4 can be considered as satisfactory. We also remark that we do not take into account $k_{\perp}$-broadening effects between HERMES and COMPASS energies and that the HERMES data actually represent multiplicities integrated (separately for numerator and denominator) over the kinematic ranges of each bin while the curve is plotted for a fixed set of kinematics. Through dedicated fits to the HERMES, COMPASS (and other) data and consideration of $k_{\perp}$-evolution effects it is possible to obtain a better description than in figure 4 , see [125].

\subsection{Leading-twist $A_{L L}$ and first test of Gaussian Ansatz in polarized scattering}

The Gaussian Ansatz is useful in unpolarized case [110-115], but nothing is known about its applicability to spin asymmetries. The JLab data [126] on $A_{L L}\left(P_{h T}\right)$ put us in the position to conduct a first "test" for polarized partons. We assume Gaussian form for $g_{1}^{a}\left(x, k_{\perp}^{2}\right)$, eq. (4.5c), and use lattice QCD results [67] to estimate the width $\left\langle k_{\perp}^{2}\right\rangle_{g_{1}}$, see appendix A.2. With $\lambda=z^{2}\left\langle k_{\perp}^{2}\right\rangle_{g_{1}}+\left\langle P_{\perp}^{2}\right\rangle_{D_{1}}$ implicit in $\mathcal{G}\left(P_{h T}\right)$, the structure function $F_{L L}$ reads

$$
\begin{aligned}
F_{L L}\left(x, z, P_{h T}\right) & =x \sum_{q} e_{q}^{2} g_{1}^{q}(x) D_{1}^{q}(z) \mathcal{G}\left(P_{h T}\right), \\
F_{L L}(x, z) & =x \sum_{q} e_{q}^{2} g_{1}^{q}(x) D_{1}^{q}(z)
\end{aligned}
$$



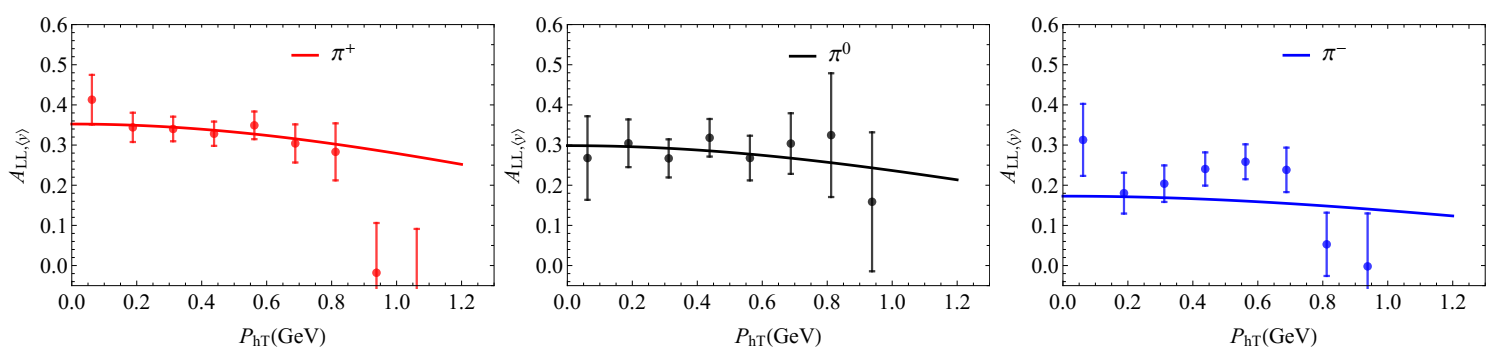

Figure 5. $A_{L L,\langle y\rangle}$ as function of $P_{h T}$ vs. JLab data [126] for $\pi^{+}, \pi^{0}, \pi^{-}$. The solid lines are our results for the mean values of kinematical variables $\langle x\rangle=0.25,\langle z\rangle=0.5$, and $\left\langle Q^{2}\right\rangle=1.67 \mathrm{GeV}^{2}$.

The definition of the asymmetry used by the JLab experiment [126] is

$$
A_{L L,\langle y\rangle}\left(x, z, P_{h T}\right)=\left\langle p_{2} A_{L L}\left(x, z, P_{h T}\right)\right\rangle=\frac{\left\langle y(2-y) F_{L L}\left(x, z, P_{h T}\right)\right\rangle}{\left\langle\left(1+(1-y)^{2}\right) F_{U U}\left(x, z, P_{h T}\right)\right\rangle},
$$

where $p_{2}=y(2-y) /\left(1+(1-y)^{2}\right)$ and averaging (separately in numerator and denominator) over the kinematics of [126] is implied. We use the lattice data [67] to constrain the Gaussian width $\left\langle k_{\perp}^{2}\right\rangle_{g_{1}}$ as described in appendix A.2. All other ingredients in (5.6) are known and tested through other observables in section 5.1. Therefore the comparison of our results to the JLab data [126] shown in figure 5 provides several important tests. First, the JLab data [126] are compatible with the Gaussian Ansatz within uncertainties. Second, the lattice results - in the way we use them in appendix A.2 - give an appropriate description of the data. (Another important test was already presented in [126]: the $P_{h T}$-integrated ("collinear") asymmetry (5.5b) is compatible with data from other experiments and theoretical results obtained from parametrizations of $f_{1}^{a}(x), g_{1}^{a}(x), D_{1}^{a}(z)$. This shows that in the pre- $12 \mathrm{GeV}$ era one was, to a good approximation, indeed already probing DIS [126].)

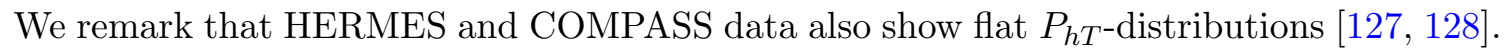

Encouraged by these findings we will use lattice predictions from ref. [67] below also for the Gaussian widths of $g_{1 T}^{\perp(1) a}$ and $h_{1 L}^{\perp(1) a}$. Of course, at this point one could argue that the WW and WW-type approximations (3.6a), (3.6b) also dictate that $g_{1 T}^{\perp}$ and $h_{1 L}^{\perp}$ have the same Gaussian widths as $g_{1}$ and $h_{1}$. In fact, the lattice results for the respective widths are numerically similar, which can be interpreted as yet another argument in favor of the usefulness of the approximations. The practical predictions depend only weakly on the choice of parameters.

\subsection{Leading-twist $A_{U T}^{\sin \left(\phi_{h}-\phi_{S}\right)}$ Sivers asymmetry}

The $F_{U T}^{\sin \left(\phi_{h}-\phi_{S}\right)}$ structure function is related to the Sivers function [129], which describes the distribution of unpolarized quarks inside a transversely polarized proton. It has so far received the widest attention, from both phenomenological and experimental points of view.

The Sivers function $f_{1 T}^{\perp}$ is related to initial and final-state interactions of the struck quark and the rest of the nucleon and could not exist without contributions of orbital angular momentum of partons to the spin of the nucleon. As such it encodes the correlation between the partonic intrinsic motion and the transverse spin of the nucleon, and it gen- 

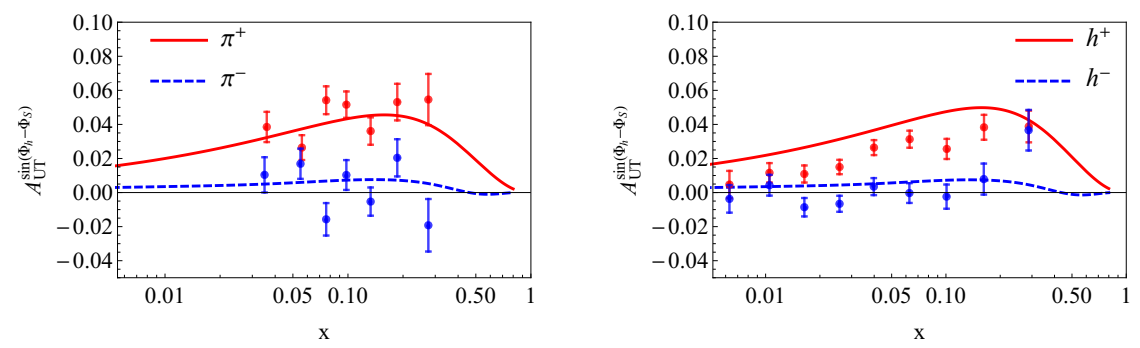

Figure 6. Sivers asymmetry $A_{U T}^{\sin \left(\phi_{h}-\phi_{S}\right)}$ for a proton target as function of $x$ based on the fit [136] in comparison to (left panel) HERMES [137] and (right panel) COMPASS [138] data.

erates a dipole deformation in momentum space. The Sivers function has been extracted from SIDIS data by several groups, with consistent results [45, 111, 130-135].

The structure function $F_{U T}^{\sin \left(\phi_{h}-\phi_{S}\right)}$ reads

$$
\begin{aligned}
F_{U T}^{\sin \left(\phi_{h}-\phi_{S}\right)}\left(x, z, P_{h T}\right) & =-x \sum_{q} e_{q}^{2} f_{1 T}^{\perp(1) q}(x) D_{1}^{q}(z) b_{\mathrm{B}}^{(1)}\left(\frac{z P_{h T}}{\lambda}\right) \mathcal{G}\left(P_{h T}\right), \\
F_{U T}^{\sin \left(\phi_{h}-\phi_{S}\right)}\left(x, z,\left\langle P_{h T}\right\rangle\right) & =-x \sum_{q} e_{q}^{2} f_{1 T}^{\perp(1) q}(x) D_{1}^{q}(z) c_{\mathrm{B}}^{(1)}\left(\frac{z}{\lambda^{1 / 2}}\right)
\end{aligned}
$$

where $\lambda=z^{2}\left\langle k_{\perp}^{2}\right\rangle_{f_{1 T}^{\perp}}+\left\langle P_{\perp}^{2}\right\rangle_{D_{1}}$ and $b_{\mathrm{B}}^{(1)}=2 M_{N}$ and $c_{\mathrm{B}}^{(1)}=\sqrt{\pi} M_{N}$, see appendix B.5 for details.

Notice that integrating structure functions over $P_{h T}$ is different from integrating the cross section over $P_{h T}$ where azimuthal hadron modulations drop out. Only if the relevant weight is $\omega^{\{0\}}$ we obtain "collinear structure functions": $F_{U U}(x, z), F_{L L}(x, z)$ in sections 5.1, 5.2, and below in sections 7.2, 7.6. In all other cases, despite integration over $P_{h T}$, we end up always with true convoluted TMDs (here within Gaussian model). We stress this important point by displaying the dependence of the structure functions on the mean transverse momentum, e.g., $F_{U T}^{\sin \left(\phi_{h}-\phi_{S}\right)}\left(x, z,\left\langle P_{h T}\right\rangle\right)=\int d^{2} P_{h T} F_{U T}^{\sin \left(\phi_{h}-\phi_{S}\right)}\left(x, z, P_{h T}\right)$ in $(5.7 \mathrm{~b})$.

The asymmetries $A_{U T}^{\sin \left(\phi_{h}-\phi_{S}\right)}=F_{U T}^{\sin \left(\phi_{h}-\phi_{S}\right)} / F_{U U}$ obtained from the fit [136] are plotted in figure 6 as functions of $x$ in comparison to HERMES [137] and COMPASS [138] data on respectively charged pion and hadron production from a proton target. Notice that the COMPASS data points seem to be below the theoretical curves which may indicate evolution effects $[48,139]$. We do not show here the description of the $P_{h T}$-dependence of the data but it is well described by the fit of [136] which confirms that the Gaussian model works also in this case. The Sivers function is predicted to enter the description of hadron-hadron collisions (with transversely polarized protons) with an opposite sign compared to SIDIS [36, 140, 141]. Recent results on single-spin asymmetries in weak-boson production from RHIC [142] and Drell-Yan from COMPASS [143, 144] are consistent with this prediction. 


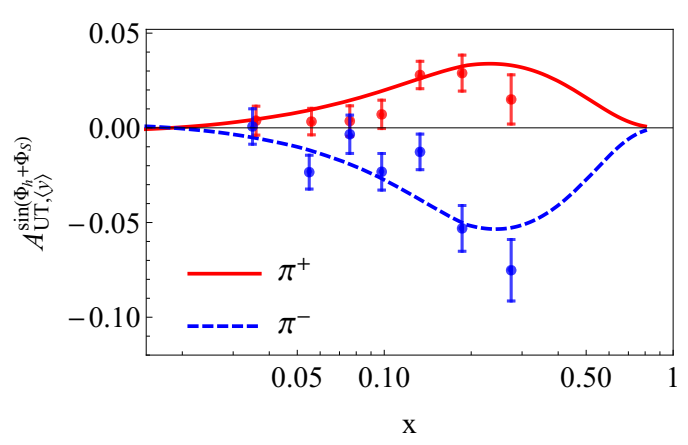

(a)

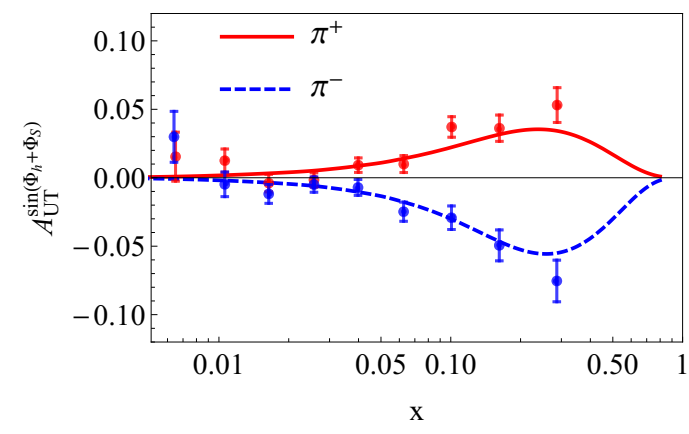

(b)

Figure 7. Collins asymmetry for a proton target vs $x$ based on the fit [152]. (a) $A_{U T,\langle y\rangle}^{\sin \left(\phi_{h}+\phi_{S}\right)}$ in comparison to HERMES [155] data. (b) $A_{U T}^{\sin \left(\phi_{h}+\phi_{S}\right)}$ in comparison to COMPASS [156] data.

\subsection{Leading-twist $A_{U T}^{\sin \left(\phi_{h}+\phi_{S}\right)}$ Collins asymmetry}

The $F_{U T}^{\sin \left(\phi_{h}+\phi_{S}\right)}$ structure function of the SIDIS cross section is due to the convolution of the transversity distribution $h_{1}$ and the Collins FF $H_{1}^{\perp}$. It describes the distribution of transversely polarized quarks in a transversely polarized nucleon, and is the only source of information on the tensor charge of the nucleon. Transversity can also be accessed as a PDF in Drell-Yan or dihadron production [145-150]. The Collins FF $H_{1}^{\perp}$ decodes the fundamental correlation between the transverse spin of a fragmenting quark and the transverse momentum of the produced final hadron [151]. There are many extractions of $h_{1}$ and $H_{1}^{\perp}$ from combined fits of SIDIS and $e^{+} e^{-}$data, for instance those of refs. [152-154]. In this work we will use the extractions of $h_{1}$ and $H_{1}^{\perp}$ from ref. [152].

The structure function $F_{U T}^{\sin \left(\phi_{h}+\phi_{S}\right)}$ reads

$$
\begin{aligned}
F_{U T}^{\sin \left(\phi_{h}+\phi_{S}\right)}\left(x, z, P_{h T}\right) & =x \sum_{q} e_{q}^{2} h_{1}^{q}(x) H_{1}^{\perp(1) q}(z) b_{\mathrm{A}}^{(1)}\left(\frac{z P_{h T}}{\lambda}\right) \mathcal{G}\left(P_{h T}\right), \\
F_{U T}^{\sin \left(\phi_{h}+\phi_{S}\right)}\left(x, z,\left\langle P_{h T}\right\rangle\right) & =x \sum_{q} e_{q}^{2} h_{1}^{q}(x) H_{1}^{\perp(1) q}(z) c_{\mathrm{A}}^{(1)}\left(\frac{z}{\lambda^{1 / 2}}\right),
\end{aligned}
$$

where $\lambda=z^{2}\left\langle k_{\perp}^{2}\right\rangle_{h_{1}}+\left\langle P_{\perp}^{2}\right\rangle_{H_{1}^{\perp}}$ and $b_{\mathrm{A}}^{(1)}=2 m_{h}$ and $c_{\mathrm{A}}^{(1)}=\sqrt{\pi} m_{h}$, see appendix B.5 for details.

The asymmetries $A_{U T,\langle y\rangle}^{\sin \left(\phi_{h}+\phi_{S}\right)}=\left\langle(1-y) F_{U T}^{\sin \left(\phi_{h}+\phi_{S}\right)}\right\rangle /\left\langle\left(1-y+y^{2} / 2\right) F_{U U}\right\rangle$ are plotted in figure 7 as functions of $x$ in comparison to HERMES [155] and $A_{U T}^{\sin \left(\phi_{h}+\phi_{S}\right)}=F_{U T}^{\sin \left(\phi_{h}+\phi_{S}\right)} / F_{U U}$ for COMPASS [156] data on charged-pion production from proton targets. We remark that the description of the $P_{h T}$-dependences of this azimuthal spin asymmetry is equally satisfactory by the fit of ref. [152], which implies that the data are compatible with the Gaussian Ansatz also in this case.

\subsection{Leading-twist $A_{U U}^{\cos \left(2 \phi_{h}\right)}$ Boer-Mulders asymmetry}

The structure function $F_{U U}^{\cos \left(2 \phi_{h}\right)}$ arises from a convolution of the Collins fragmention function and the Boer-Mulders TMD $h_{1}^{\perp}$, which describes the distribution of transversely po- 

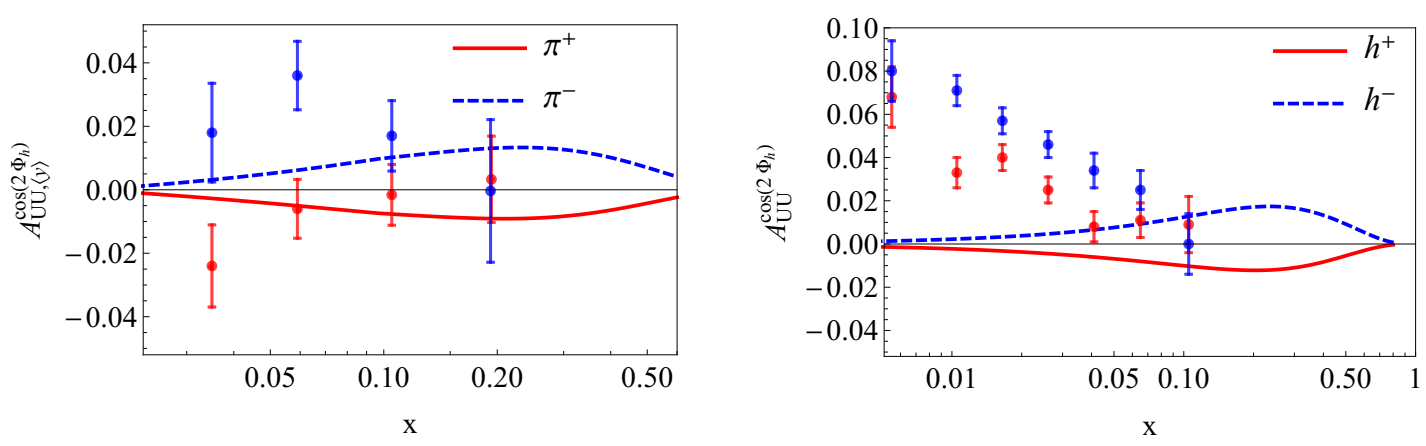

Figure 8. The asymmetry $A_{U U,\langle y\rangle}^{\cos \left(2 \phi_{h}\right)}$ for a proton target as function of $x$ based on the fit [157] in comparison to (left panel) HERMES [158] and $A_{U U}^{\cos \left(2 \phi_{h}\right)}$ (right panel) COMPASS data [159].

larized partons inside an unpolarized target. The expression of this structure function is given by

$$
\begin{aligned}
F_{U U}^{\cos \left(2 \phi_{h}\right)}\left(x, z, P_{h T}\right) & =x \sum_{q} e_{q}^{2} h_{1}^{\perp(1) q}(x) H_{1}^{\perp(1) q}(z) b_{\mathrm{AB}}^{(2)}\left(\frac{z P_{h T}}{\lambda}\right)^{2} \mathcal{G}\left(P_{h T}\right), \\
F_{U U}^{\cos 2 \phi_{h}}\left(x, z,\left\langle P_{h T}\right\rangle\right) & =x \sum_{q} e_{q}^{2} h_{1}^{\perp(1) q}(x) H_{1}^{\perp(1) q}(z) c_{\mathrm{AB}}^{(2)}\left(\frac{z}{\lambda^{1 / 2}}\right)^{2},
\end{aligned}
$$

where $\lambda=z^{2}\left\langle k_{\perp}^{2}\right\rangle_{h_{1}^{\perp}}+\left\langle P_{\perp}^{2}\right\rangle_{H_{1}^{\perp}}$ and $b_{\mathrm{AB}}^{(2)}=4 M_{N} m_{h}$ and $c_{\mathrm{AB}}^{(2)}=4 M_{N} m_{h}$, see appendix B.5.

The asymmetries $A_{U U,\langle y\rangle}^{\cos \left(2 \phi_{h}\right)}=\left\langle(1-y) F_{U U}^{\cos \left(2 \phi_{h}\right)}\right\rangle /\left\langle\left(1-y+y^{2} / 2\right) F_{U U}\right\rangle$ for HERMES [158] and $A_{U U}^{\cos \left(2 \phi_{h}\right)}=F_{U U}^{\cos \left(2 \phi_{h}\right)} / F_{U U}$ for COMPASS [159] are plotted in figure 8, where we only considered the Boer-Mulders contribution to $A_{U U}^{\cos \left(2 \phi_{h}\right)}$, which does not describe the data well. Especially for COMPASS one can see that calculation and data are of opposite signs. In fact, it is suspected that this observable receives a significant contribution from the Cahn effect [160], a term of higher-twist character of the type $\left\langle P_{h T}^{2}\right\rangle / Q^{2}$, which is not negligible in fixed-target experiments as shown in phenomenological [113] and model [161] studies. In the phenomenological works $[157,162,163]$, an attempt was made to estimate and correct for this contribution in order to obtain a picture of the Boer-Mulders function undistorted from Cahn effect. The point is that this substantial twist-4 contamination can be estimated phenomenologically, even though there is no rigorous theoretical basis for the description of such power-suppressed terms. In this work we consistently neglect power-suppressed contributions of order $1 / Q^{2}$, and do so also in figure 8 . Nevertheless, we of course use the parametrizations of $[157,162,163]$ offering the best currently available parametrizations for $h_{1}^{\perp}$, which were corrected for the Cahn effect as good as it is possible at the current state of art. It is unknown whether other asymmetries could be similarly effected by such type of power corrections. This is an important point to be kept in mind as the lesson from figure 8 shows. 


\subsection{Leading-twist $A_{U T}^{\sin \left(3 \phi_{h}-\phi_{S}\right)}$ asymmetry}

The pretzelosity TMD $h_{1 T}^{\perp q}$ is the least known basis function. It is of interest as it allows one to measure the deviation of the nucleon spin density from spherical shape [12], is related to the only leading-twist SIDIS structure function where the small- $P_{h T}$ description in terms of TMDs and the large- $P_{h T}$ expansion in perturbative QCD mismatch [116], and is the only TMD where a clear relation to quark orbital angular momentum could be established (albeit only within quark models) [85, 164-166].

The structure function $F_{U T}^{\sin \left(3 \phi_{h}-\phi_{S}\right)}$ reads

$$
\begin{aligned}
F_{U T}^{\sin \left(3 \phi_{h}-\phi_{S}\right)}\left(x, z, P_{h T}\right) & =x \sum_{q} e_{q}^{2} h_{1 T}^{\perp(2) q}(x) H_{1}^{\perp(1) q}(z) b^{(3)}\left(\frac{z P_{h T}}{\lambda}\right)^{3} \mathcal{G}\left(P_{h T}\right), \\
F_{U T}^{\sin \left(3 \phi_{h}-\phi_{S}\right)}\left(x, z,\left\langle P_{h T}\right\rangle\right) & =x \sum_{q} e_{q}^{2} h_{1 T}^{\perp(2) q}(x) H_{1}^{\perp(1) q}(z) c^{(3)}\left(\frac{z}{\lambda^{1 / 2}}\right)^{3}
\end{aligned}
$$

where $\lambda=z^{2}\left\langle k_{\perp}^{2}\right\rangle_{h_{1 T}^{\perp}}+\left\langle P_{\perp}^{2}\right\rangle_{H_{1}^{\perp}}$ and $b^{(3)}=2 M_{N}^{2} m_{h}$ and $c^{(3)}=3 / 2 \sqrt{\pi} M_{N}^{2} m_{h}$, see appendix B.5. In eq. (5.10a) we see that this structure function suffers a cubic suppression for small transverse hadron momenta.

Preliminary COMPASS data [167] for $A_{U T}^{\sin \left(3 \phi_{h}-\phi_{S}\right)}=F_{U T}^{\sin \left(3 \phi_{h}-\phi_{S}\right)} / F_{U U}$ and preliminary HERMES data [168] for $A_{U T,\langle y\rangle}^{\sin \left(3 \phi_{h}-\phi_{S}\right)}=\left\langle(1-y) F_{U T}^{\sin \left(3 \phi_{h}-\phi_{S}\right)}\right\rangle /\left\langle\left(1-y+y^{2} / 2\right) F_{U U}\right\rangle$ are plotted in figure 9. Clearly, the pretzelosity TMD is the least known of the basis TMDs. Nevertheless it is of fundamental importance, as it provides one of the basis functions in our approach. It is so difficult to access it experimentally, because its contribution to the SIDIS cross section is proportional to $P_{h T}^{3}$, the TMD approach requires us to necessarily operate at $P_{h T} \ll Q$, and so far only moderate values of $Q$ could be achieved in the fixed target experiments. Future high-luminosity data from JLab 12 are expected to significantly improve our knowledge of this TMD.

For comparison figure 15 shows also the results from the quark-diquark model calculation of ref. [170], and from the light-cone constituent quark model of ref. [171].

\subsection{Statistical and systematic uncertainties of basis functions}

Even the well-known collinear functions $f_{1}^{a}(x)$ and $g_{1}^{a}(x)$ have statistical uncertainties and systematic uncertainties (the latter introduced by choosing a certain fit Ansatz, which however can be avoided through neural-network techniques [172]). These uncertainties as well as those of $D_{1}^{a}(z)$ can safely be neglected for our purposes. For TMDs the situation is different. Already the transverse momentum descriptions of $f_{1}^{a}\left(x, k_{\perp}^{2}\right)$ and $D_{1}^{a}\left(z, P_{\perp}^{2}\right)$ are associated with non-negligible statistical uncertainties, which are reviewed in appendix A, and with systematic uncertainties that are very difficult to assess as they are related to model bias (because of Gaussian model and its limitations). The statistical and systematic uncertainties are significant when we deal with the basis functions $f_{1 T}^{\perp q}, h_{1}^{q}, H_{1}^{\perp q}$. The least well-controlled uncertainties are associated with the Boer-Mulders function $h_{1}^{\perp q}$ and pretzelosity $h_{1 T}^{\perp q}$.

In this work we are not interested in these uncertainties, which future data will allow us to diminish, even though in practice they may be sizable. Rather in this work we 


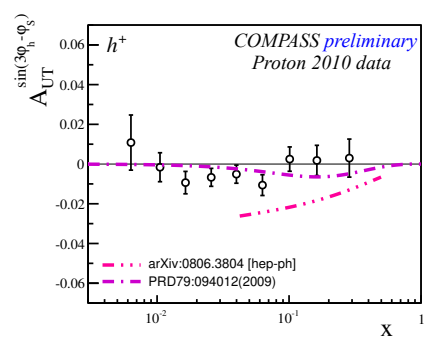

(a)

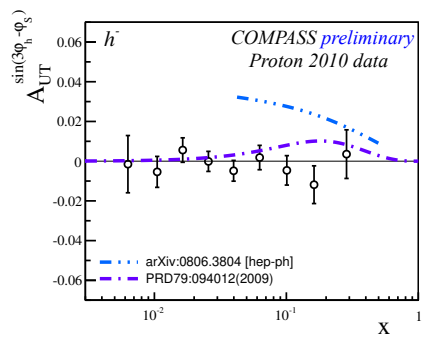

(b)

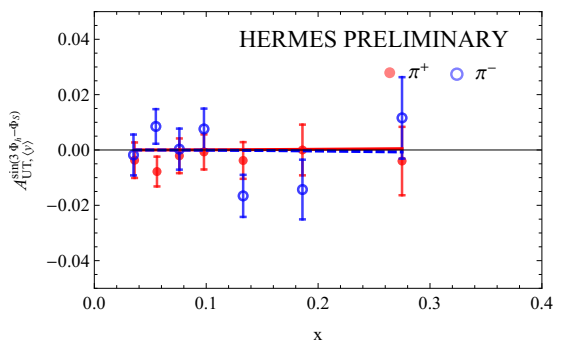

(c)

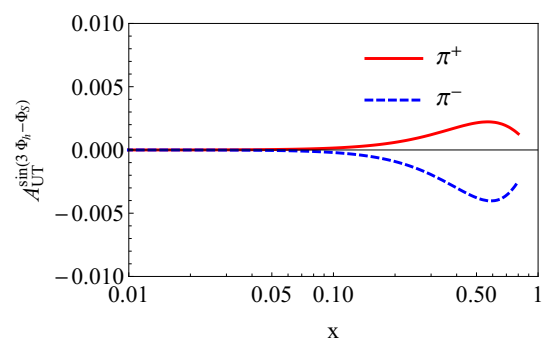

(d)

Figure 9. $A_{U T}^{\sin \left(3 \phi_{h}-\phi_{S}\right)}$ as a function of $x$ from preliminary COMPASS [167] (a,b) and $A_{U T,\langle y\rangle}^{\sin \left(3 \phi_{h}-\phi_{S}\right)}$ for HERMES [168] (c) in comparison to the best fit from [169] (whose 1- $\sigma$ uncertainty band is compatible with zero), and our calculation for COMPASS kinematics (d). For comparison the COMPASS plots show the model results $[170,171]$. [We remark that in this and several subsequent figures we have the permission to show the preliminary data [167] only in the official figures provided by the COMPASS collaboration in (a,b), and have to display our results separately in (d). Notice also the different scale on the y-axis in panel (d) as compared to (a,b).]

are interested in making projections based on the WW-type approximation. To avoid cumbersome and difficult-to-interpret figures, we will therefore refrain from indicating the uncertainties associated with our current knowledge of the basis functions. In the following we will only display the estimated systematic uncertainty associated with the WW-type approximations assuming it works within a relative accuracy of $\pm 40 \%$. We stress that this is only to simplify the presentation. The actual uncertainty of the predictions may be larger.

\section{Leading-twist asymmetries in WW-type approximation}

Two out of the eight leading-twist structure functions can be described in the WW-type approximation thanks to eqs. (3.6a), (3.6b), which relate $g_{1 T}^{\perp(1) q}(x)$ and $h_{1 L}^{\perp(1) q}(x)$ to the basis functions $g_{1}^{q}(x)$ and $h_{1}^{a}(x)$, see figure 10. These TMDs are sometimes referred to as "worm gear" functions, where $g_{1 T}^{\perp q}$ describes the distributions of longitudinally polarized quarks inside a transversely polarized nucleon, and $h_{1 L}^{\perp q}$ the opposite configuration, namely transversely polarized quarks inside a longitudinally polarized nucleon. It is interesting that both cases can be expressed in the WW-type approximation in terms of the basis functions. In this section we discuss the associated asymmetries. 

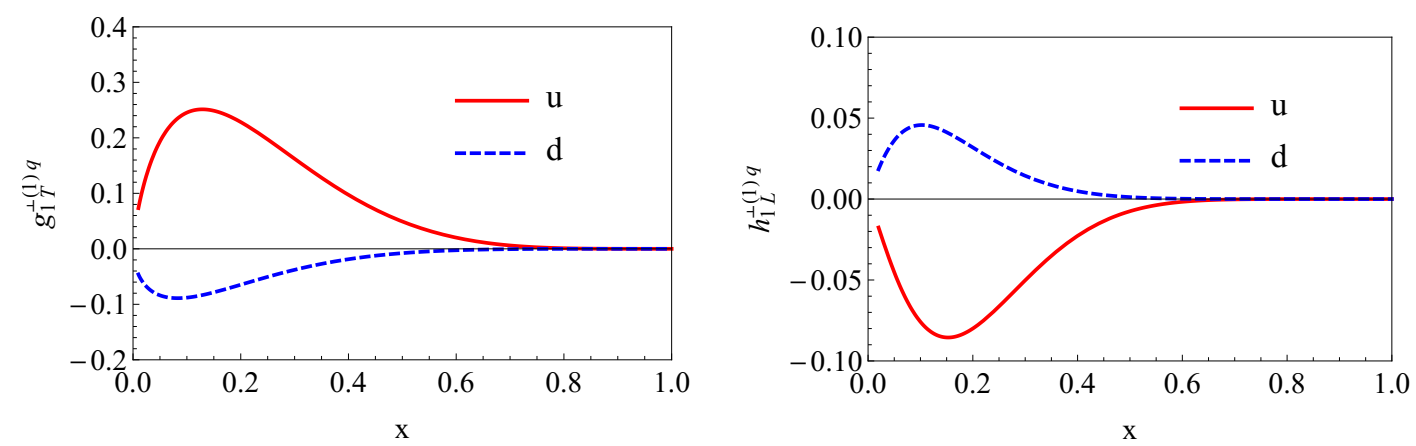

Figure 10. $g_{1 T}^{\perp(1) q}(x)$ (left panel) and $h_{1 L}^{\perp(1) q}(x)$ distributions (right panel) for up and down flavor as predicted by the WW-type approximations in eqs. (3.6a), (3.6b).

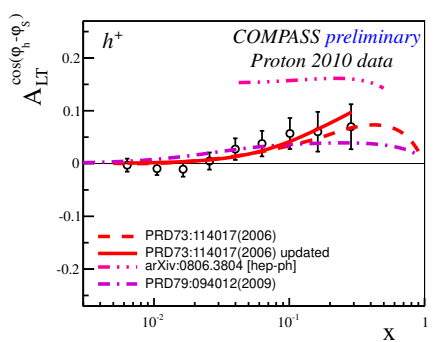

(a)

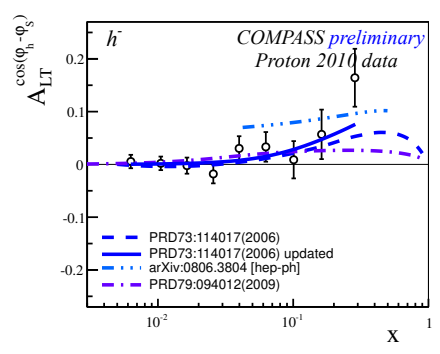

(b)

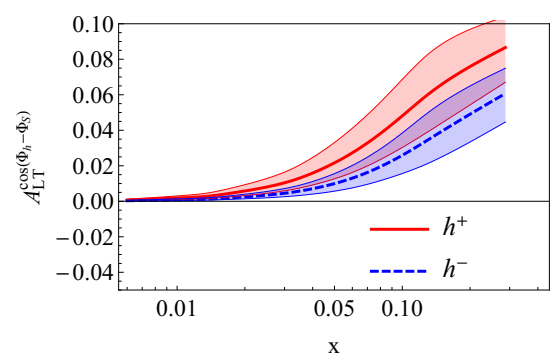

(c)

Figure 11. Leading-twist $A_{L T}^{\cos \left(\phi_{h}-\phi_{S}\right)}$ : preliminary COMPASS data [167] (a,b); and our calculation for COMPASS kinematics (c) shown separately for reasons explained in the caption of figure 9.

\subsection{Leading-twist $A_{L T}^{\cos \left(\phi_{h}-\phi_{S}\right)}$}

We assume for $g_{1 T}^{\perp}$ the Gaussian Ansatz as shown in (B.9a) of appendix B.3, see also [28], and evaluate $g_{1 T}^{\perp(1) q}(x)$ using (3.6a), which yields the result shown in figure 10. For our numerical estimates we use $\left\langle k_{\perp}^{2}\right\rangle_{g_{1 T}^{\perp}}=\left\langle k_{\perp}^{2}\right\rangle_{g_{1}}$, which is supported by lattice results [67].

In the Gaussian Ansatz the structure function $F_{L T}^{\cos \left(\phi_{h}-\phi_{S}\right)}$ has the form

$$
\begin{aligned}
F_{L T}^{\cos \left(\phi_{h}-\phi_{S}\right)}\left(x, z, P_{h T}\right) & =x \sum_{q} e_{q}^{2} g_{1 T}^{\perp(1) q}(x) D_{1}^{q}(z) b_{\mathrm{B}}^{(1)}\left(\frac{z P_{h T}}{\lambda}\right) \mathcal{G}\left(P_{h T}\right) \\
F_{L T}^{\cos \left(\phi_{h}-\phi_{S}\right)}\left(x, z,\left\langle P_{h T}\right\rangle\right) & =x \sum_{q} e_{q}^{2} g_{1 T}^{\perp(1) q}(x) D_{1}^{q}(z) c_{\mathrm{B}}^{(1)}\left(\frac{z}{\lambda^{1 / 2}}\right)
\end{aligned}
$$

where $\lambda=z^{2}\left\langle k_{\perp}^{2}\right\rangle_{g_{1 T}^{\perp}}+\left\langle P_{\perp}^{2}\right\rangle_{D_{1}}, b_{\mathrm{B}}^{(1)}=2 M_{N}, c_{\mathrm{B}}^{(1)}=\sqrt{\pi} M_{N}$, see appendix B.5 for details.

This asymmetry was measured at JLab [173], COMPASS [174-176] and HERMES [177, 178] (for the latter two experiments only preliminary results are available so far). Figure 11 shows the preliminary results from the 2010 COMPASS data [167], in addition to our calculation, where we approximate the charged hadrons (70-80\% of which are $\pi^{ \pm}$at COMPASS) by charged pions, see appendix A.1. We observe that the WW-type approximation describes the data within their experimental uncertainties. For comparison also results from the theoretical works $[28,170,171]$ are shown. Our results are also compatible with the 


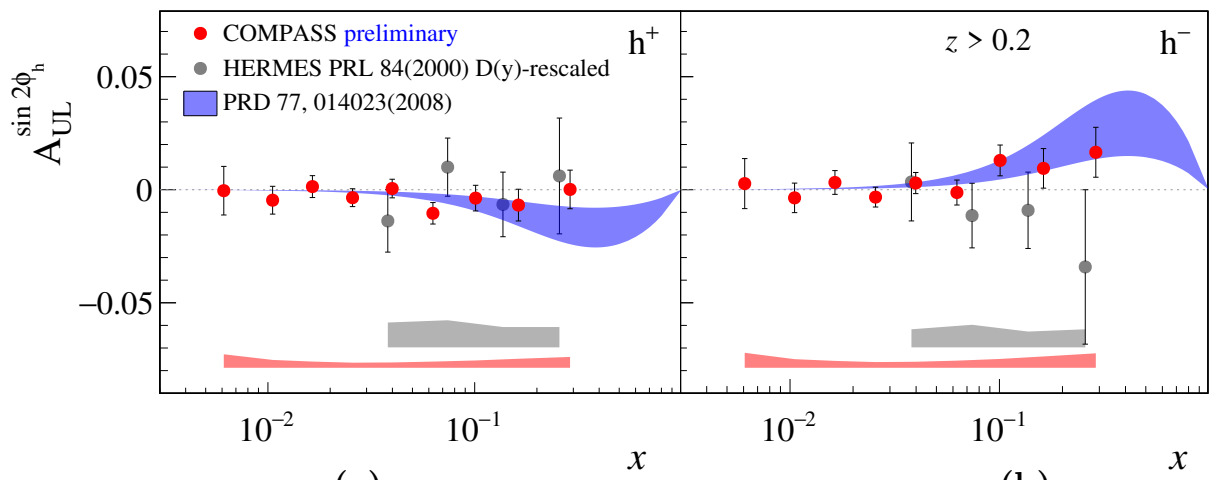

(a)

(b)

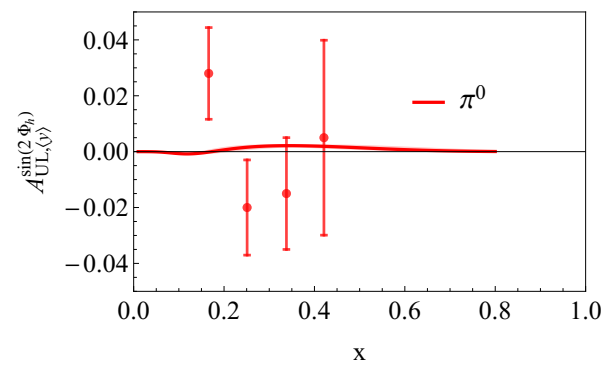

(c)

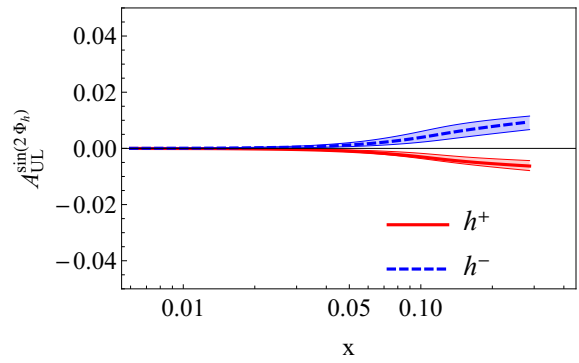

(d)

Figure 12. Leading twist $A_{U L}^{\sin \left(2 \phi_{h}\right)}$ vs. $x$ from HERMES [179] and COMPASS [176, 180] (a, b), $A_{U L,\langle y\rangle}^{\sin \left(2 \phi_{h}\right)}$ from JLab [181] (c), and from our calculation for COMPASS kinematics in the WWtype approximation (d) shown separately for reasons explained in the caption of figure 9 . Results from [29] are also shown in comparison to the COMPASS data.

JLab data, which were taken with a neutron $\left({ }^{3} \mathrm{He}\right)$ target [173] and have larger statistical uncertainties than the preliminary COMPASS and HERMES data.

\subsection{Leading-twist $A_{U L}^{\sin 2 \phi_{h}}$ Kotzinian-Mulders asymmetry}

We use the Gaussian form for the Kotzinian-Mulders function $h_{1 L}^{\perp a}$, (B.9b) in appendix B.3, with $\left\langle k_{\perp}^{2}\right\rangle_{h_{1 L}^{\perp}}=\left\langle k_{\perp}^{2}\right\rangle_{h_{1}}$ as supported by lattice data [67]. From (3.6b) we obtain the WWtype estimate for $h_{1 L}^{\perp(1) a}(x)$ shown in figure 10. The structure function $F_{U L}^{\sin \left(2 \phi_{h}\right)}$ reads

$$
\begin{aligned}
F_{U L}^{\sin \left(2 \phi_{h}\right)}\left(x, z, P_{h T}\right) & =x \sum_{q} e_{q}^{2} h_{1 L}^{\perp(1) q}(x) H_{1}^{\perp(1) q / h}(z)\left(\frac{z P_{h T}}{\lambda}\right)^{2} b_{\mathrm{AB}}^{(2)} \mathcal{G}\left(P_{h T}\right), \\
F_{U L}^{\sin \left(2 \phi_{h}\right)}\left(x, z,\left\langle P_{h T}\right\rangle\right) & =x \sum_{q} e_{q}^{2} h_{1 L}^{\perp(1) q}(x) H_{1}^{\perp(1) q / h}(z)\left(\frac{z}{\lambda^{1 / 2}}\right)^{2} c_{\mathrm{AB}}^{(2)}
\end{aligned}
$$

where $\lambda=z^{2}\left\langle k_{\perp}^{2}\right\rangle_{h_{1 L}^{\perp}}+\left\langle P_{\perp}^{2}\right\rangle_{H_{1}^{\perp}}$ and $b_{\mathrm{AB}}^{(2)}=c_{\mathrm{AB}}^{(2)}=4 M_{N} m_{h}$, see appendix B.5 for details.

The asymmetry $A_{U L}^{\sin \left(2 \phi_{h}\right)}=F_{U L}^{\sin \left(2 \phi_{h}\right)} / F_{U U}$ was studied at HERMES [179, 182], COMPASS [176, 180], and JLab [126, 181]. In figure 12 proton data are shown for $\pi^{ \pm}$ in the HERMES experiment measured with the $27.6 \mathrm{GeV}$ positron beam of HERA for 
$1 \mathrm{GeV}^{2}<Q^{2}<15 \mathrm{GeV}^{2}, W>2 \mathrm{GeV}, 0.023<x<0.4$, and $y<0.85$. The COMPASS data were taken in $2007(160 \mathrm{GeV})$ and $2011(200 \mathrm{GeV})$ and show the asymmetry for charged hadrons (in practice mainly pions). Since $y$-dependent prefactors were included in the analyses (see section 2.1), the HERMES data are adequately (" $D(y)-$ ")rescaled. The CLAS $\pi^{0}$ data in the right panel were measured using $6 \mathrm{GeV}$ longitudinally polarized electrons scattering off longitudinally polarized protons in a cryogenic ${ }^{14} \mathrm{NH}_{3}$ target in the kinematic region of $1.0 \mathrm{GeV}^{2}<Q^{2}<3.2 \mathrm{GeV}^{2}, 0.12<x<0.48$ and $0.4<z<0.7$ [181].

$A_{U L}^{\sin \left(2 \phi_{h}\right)}$ can be expected to be smaller than $A_{L T}^{\cos \left(\phi_{h}-\phi_{S}\right)}$ discussed in section 6.1, even though both are leading twist. This is because $F_{U L}^{\sin \left(2 \phi_{h}\right)}$ is quadratic in the hadron transverse momenta $P_{h T}$, while $F_{L T}^{\cos \left(\phi_{h}-\phi_{S}\right)}$ is linear. In addition, the former is proportional to the Collins function, which is smaller than $D_{1}^{q}(z)$, and the WW-type approximation predicts the magnitude of $h_{1 L}^{\perp(1) q}(x)$ to be about half of the size of $g_{1 T}^{\perp(1) q}(x)$. The data support these expectations. HERMES and JLab data are compatible with zero for this asymmetry. So are the preliminary COMPASS data except for the region $x>0.1$ for negative hadrons, where the trend of the data provides a first encouraging confirmation for our results. Thus current data are compatible with the WW-type approximation for $h_{1 L}^{\perp(1) q}(x)$.

\subsection{Inequalities and a cross check}

We discussed WW-type approximations for the twist-2 TMDs $g_{1 T}^{\perp q}$ and $h_{1 L}^{\perp q}$ in sections 6.1, 6.2. Before proceeding with twist-3 let us pause and revisit positivity bounds [15].

The Kotzinian-Mulders function $h_{1 L}^{\perp q}$ in conjunction with the Boer-Mulders function, and the TMD $g_{1 T}^{\perp q}$ in conjunction with the Sivers function obey the positivity bounds [15]

$$
\begin{aligned}
& \frac{k_{\perp}^{2}}{4 M_{N}^{2}}\left(\left(f_{1}^{q}\left(x, k_{\perp}^{2}\right)\right)^{2}-\left(g_{1}^{q}\left(x, k_{\perp}^{2}\right)\right)^{2}\right)-\left(h_{1 L}^{\perp(1) q}\left(x, k_{\perp}^{2}\right)\right)^{2}-\left(h_{1}^{\perp(1) q}\left(x, k_{\perp}^{2}\right)\right)^{2} \geq 0, \\
& \frac{k_{\perp}^{2}}{4 M_{N}^{2}}\left(\left(f_{1}^{q}\left(x, k_{\perp}^{2}\right)\right)^{2}-\left(g_{1}^{q}\left(x, k_{\perp}^{2}\right)\right)^{2}\right)-\left(f_{1 T}^{\perp(1) q}\left(x, k_{\perp}^{2}\right)\right)^{2}-\left(g_{1 T}^{\perp(1) q}\left(x, k_{\perp}^{2}\right)\right)^{2} \geq 0,
\end{aligned}
$$

where $f^{(1)}\left(x, k_{\perp}^{2}\right) \equiv \frac{k_{\perp}^{2}}{2 M_{N}^{2}} f\left(x, k_{\perp}^{2}\right)$. The inequalities provide a non-trivial test of our approach. The inequalities (6.3a), (6.3b) must be strictly satisfied by the exact leading-order QCD expressions for the TMDs. (For PDFs it is known that positivity can be preserved in some schemes and violated in others. For TMDs not much is known about positivity conditions beyond leading order.) However, here we do not deal with exact TMDs but (i) we invoked strong model assumptions (WW-type approximations for $g_{1 T}^{\perp q}$ and $h_{1 L}^{\perp q}$ and Gaussian Ansatz for all TMDs), and (ii) we deal with first extractions, which have sizable uncertainties including poorly controlled systematic uncertainties. Therefore, considering that we deal with approximations (WW-type, Gauss) and considering the current state of TMD extractions, the inequalities (6.3a), (6.3b) constitute a challenging test for the approach.

In order to conduct a test we use the Gaussian Ansatz (4.5a), (4.5d), (4.5f), (4.5g), (B.9a), (B.9b) for the TMDs and integrate over $k_{\perp}$. The results are shown in figure 13, where we plot the "normalized inequalities" defined as follows: given an inequality $a-b-$ $\cdots \geq 0$, the normalized inequality is defined as: $0 \leq(a-b-\ldots) /(|a|+|b|+\ldots) \leq 1$. 

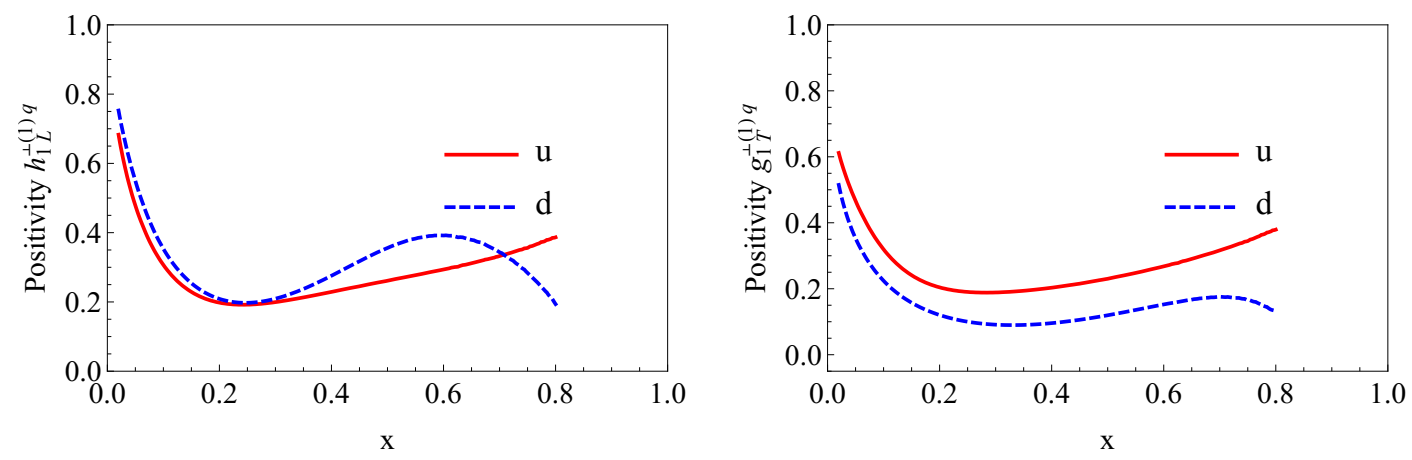

Figure 13. The normalized inequalities for $g_{1 T}^{\perp(1) q}(x)$ and $h_{1 L}^{\perp(1) q}(x)$ vs. $x$ which are obtained by integrating (6.3a) and (6.3b) over $k_{\perp}$ and normalizing with respect to the sum of the absolute values of the individual terms. The result must be positive and smaller than unity if the WW-type approximations and the application of the Gaussian model are compatible with positivity, see text. Clearly, our approach respects this test of the positivity conditions.

Figure 13 shows that the results of our approach for the "normalized inequalities" for both TMDs lie between zero and one, as it is dictated by positivity constraints. This is an important consistency cross-check for our approach.

\section{Subleading-twist asymmetries in WW-type approximation}

WW-type approximations can be applied to all eight twist-3 asymmetries, see section 4.2. In this section we discuss all of them, starting with less complex cases and proceeding then to those structure functions whose description in WW-type approximation is more involved.

\subsection{Subleading-twist $A_{L U}^{\sin \phi_{h}}$}

We start our discussion with the structure function $F_{L U}^{\sin \phi_{h}}$, eq. (2.18b), containing four terms: two are proportional to the pure twist- 3 fragmentation functions $\tilde{G}^{\perp a}$ and $\tilde{E}^{a}$; the other two terms are proportional to the twist-3 TMDs $e^{a}$ and $g^{\perp a}$, which also turn out to be given in terms of pure twist-3 terms upon the inspection of (3.3a), (3.4d). Hence, after consequently applying the WW-type approximation, we are left with no term. Our approximation predicts this structure function to be zero.

In this asymmetry we encounter the generic limitation of the WW-type approximation in most extreme form. As discussed in section 3.8, if we have a function $=\langle\bar{q} q\rangle+\langle\bar{q} g q\rangle$ the necessary condition for the approximation to work is that $\langle\bar{q} q\rangle \neq 0$ and the sufficient condition would be $|\langle\bar{q} q\rangle| \gg|\langle\bar{q} g q\rangle|$. Remarkably, none of the twist-3 TMDs or FFs entering this structure function satisfy even the necessary condition. In this situation we do not expect the WW-type approximation to work.

Indeed, data from COMPASS, HERMES, and JLab show a clearly non-zero asymmetry $A_{L U}^{\sin \phi_{h}}=F_{L U}^{\sin \phi_{h}} / F_{U U}$ of the order of $2 \%[159,183-187]$ (which includes the $1 / Q$ factor intrinsic in twist-3 observables; without this factor the asymmetry would reach $10 \%$ at large $x$ at COMPASS). This observable provides a unique opportunity to learn about the physics of $\bar{q} g q$-terms, but is beyond the applicability of the WW-type approximation. 
With the numerator of the asymmetry proportional to $\bar{q} g q$-terms and the denominator given in terms of $\bar{q} q$-terms, one could be tempted to interpret this observation as

$$
A_{L U}^{\sin \phi_{h}} \propto \frac{\langle\bar{q} g q\rangle}{\langle\bar{q} q\rangle} \stackrel{\exp }{\sim} \mathcal{O}(2 \%) .
$$

Thus, in some sense the observed effect hints at the smallness of the involved $\bar{q} g q$-terms. While in principle a correct observation, one should keep in mind several reservations. First, the experimental result (7.1) contains kinematic prefactors, which help to reduce the value. Second, the denominator contains $f_{1}^{a}$ and $D_{1}^{a}$, which are the largest TMD and FF because of positivity constraints. Third, the numerator is a sum of four terms, so its overall smallness could result from cancellation of different terms, rather than indicating that each single $\bar{q} g q$-term is small. Fourth, some asymmetries predicted to be non-zero in WW-approximation are not larger and in some cases even smaller than the result in (7.1).

To conclude, the WW-type approximation predicts $A_{L U}^{\sin \phi} \approx 0$ in contradiction to experiment. The size of the observed effect seems in line with the $\mathrm{WW}$-type approximation, as $A_{L U}^{\sin \phi_{h}} \sim\langle\bar{q} g q\rangle /\langle\bar{q} q\rangle \sim \mathcal{O}(2 \%)$ is not large, although this interpretation has reservations. $F_{L U}^{\sin \phi}$ is the only twist-3 SIDIS structure function not "contaminated" by leading twist. Attempts to describe this observable and relevant model studies have been reported [34, 54, 93, 98-100, 188-198]. But more phenomenological work and dedicated studies on the basis of models of $\bar{q} g q$ terms are needed to fully understand this asymmetry.

\subsection{Subleading-twist $A_{L T}^{\cos \phi_{S}}$}

In the WW-type approximation the structure function $F_{L T}^{\cos \phi_{S}}$ arises from $g_{T}^{a}\left(x, k_{\perp}^{2}\right)$ and $D_{1}^{a}\left(z, P_{\perp}^{2}\right)$, whose collinear counterparts are more or less known, see sections 3.4 and 5.1. We assume the Gaussian Ansatz for $g_{T}^{a}\left(x, k_{\perp}^{2}\right)$, shown in (B.9c) of appendix B.3, with $\left\langle k_{\perp}^{2}\right\rangle_{g_{T}}=\left\langle k_{\perp}^{2}\right\rangle_{g_{1}}$. We then determine $g_{T}^{q}(x)$ according to (3.2a), which is a well-tested approximation in DIS, see section 3.4. In this way we obtain for $F_{L T}^{\cos \phi_{S}}$ the result

$$
\begin{aligned}
F_{L T}^{\cos \phi_{S}}\left(x, z, P_{h T}\right) & =-\frac{2 M_{N}}{Q} x^{2} \sum_{q} e_{q}^{2} g_{T}^{q}(x) D_{1}^{q}(z) \mathcal{G}\left(P_{h T}\right), \\
F_{L T}^{\cos \phi_{S}}(x, z) & =-\frac{2 M_{N}}{Q} x^{2} \sum_{q} e_{q}^{2} g_{T}^{q}(x) D_{1}^{q}(z)
\end{aligned}
$$

with the width $\lambda=z^{2}\left\langle k_{\perp}^{2}\right\rangle_{g_{T}}+\left\langle P_{\perp}^{2}\right\rangle_{D_{1}}$ in the Gaussian $\mathcal{G}\left(P_{h T}\right)$.

Notice that we followed here the scheme explained in section 4.4: first assume Gaussian Ansatz, then apply WW-type approximation. For some structure functions the order of these steps is not relevant, but here it is. It is instructive to discuss what the opposite order of these steps would yield. Using first the WW-type approximation (3.3e) in the convolution integral (3.2a) and then using the Gaussian Ansatz yields a bulkier analytical expression than (7.2a) though a numerically similar result. But there are two critical issues with that. First, the WW-type approximation (3.3e) relates $x g_{T}^{q}\left(x, k_{\perp}^{2}\right)=g_{1 T}^{\perp(1) q}\left(x, k_{\perp}^{2}\right)$ which would imply $g_{T}^{q}\left(x, k_{\perp}^{2}\right) \rightarrow 0$ for $k_{\perp} \rightarrow 0$ due to the weight $k_{\perp}^{2} /\left(2 M_{N}^{2}\right)$ in the (1)-moment, which is not supported by model calculations [85]. Second, the more economic (because less 


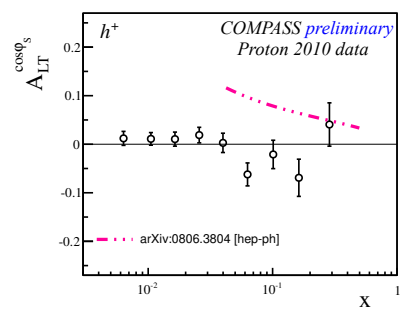

(a)

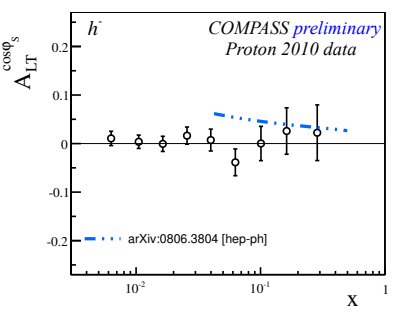

(b)

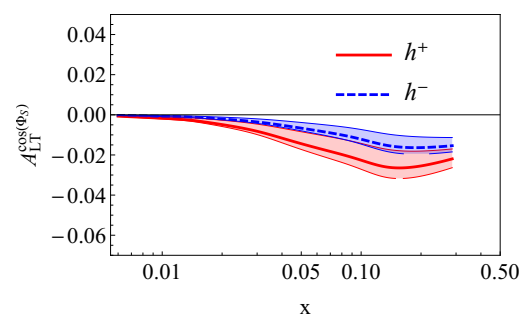

(c)

Figure 14. Subleading-twist asymmetry $A_{L T}^{\cos \phi_{S}}$ as function of $x$ from scattering of $160 \mathrm{GeV}$ longitudinally polarized muons off a transversely polarized proton target [167] (a,b), and our calculation for COMPASS kinematics (c) where a different scale is chosen to better visualize the theory curves.

bulky) expression in (7.2a) automatically yields (7.2b), which is the correct collinear result for this SIDIS structure function in (2.20c) in WW-type approximation. This technical remark confirms the consistency of the scheme suggested in section 4.4.

Figure 14 shows our predictions for $A_{L T}^{\cos \phi_{S}}$ in comparison to preliminary COMPASS data [167]. The predicted asymmetry is small and compatible with the COMPASS data within uncertainties. Preliminary HERMES data [178] confirm a small asymmetry. More precise data are necessary to judge how well the WW-type approximation works in this case. Such data could come from the JLab12 experiments. For further model studies of this asymmetry we refer to refs. [199, 200].

\subsection{Subleading-twist $A_{L T}^{\cos \left(2 \phi_{h}-\phi_{S}\right)}$}

In the WW-type approximation this asymmetry is expressed in terms of $g_{T}^{\perp a}\left(x, k_{\perp}^{2}\right)$, for which we assume a Gaussian Ansatz according to (B.9d) in appendix B.3, and use the WW-type approximation (3.3d) as

$$
x g_{T}^{\perp(2) q}(x)=\frac{\left\langle k_{\perp}^{2}\right\rangle_{g_{1 T}^{\perp}}}{M_{N}^{2}} g_{1 T}^{\perp(1) q}(x),
$$

where we finally express $g_{1 T}^{\perp(1) q}(x)$ in terms of $g_{1}^{q}(x)$ according to eq. (3.6a). For the Gaussian widths we assume $\left\langle k_{\perp}^{2}\right\rangle_{g_{T}^{\perp}}=\left\langle k_{\perp}^{2}\right\rangle_{g_{1 T}^{\perp}}=\left\langle k_{\perp}^{2}\right\rangle_{g_{1}}$. This yields for the structure function

$$
\begin{aligned}
F_{L T}^{\cos \left(2 \phi_{h}-\phi_{S}\right)}\left(x, z, P_{h T}\right) & =-\frac{2 M_{N}}{Q} x \sum_{q} e_{q}^{2} x g_{T}^{\perp(2) q}(x) D_{1}^{q}(z) b_{C}^{(2)}\left(\frac{z P_{h T}}{\lambda}\right)^{2} \mathcal{G}\left(P_{h T}\right), \\
F_{L T}^{\cos \left(2 \phi_{h}-\phi_{S}\right)}\left(x, z,\left\langle P_{h T}\right\rangle\right) & =-\frac{2 M_{N}}{Q} x \sum_{q} e_{q}^{2} x g_{T}^{\perp(2) q}(x) D_{1}^{q}(z) c_{C}^{(2)}\left(\frac{z}{\lambda^{1 / 2}}\right)^{2}
\end{aligned}
$$

where $\lambda=z^{2}\left\langle k_{\perp}^{2}\right\rangle_{g_{T}}+\left\langle P_{\perp}^{2}\right\rangle_{D_{1}}$ and $b_{\mathrm{C}}^{(2)}=c_{\mathrm{C}}^{(2)}=M_{N}^{2}$, see appendix B.5 for details.

Our predictions for the asymmetry $A_{L T}^{\cos \left(2 \phi_{h}-\phi_{S}\right)}=F_{L T}^{\cos \left(2 \phi_{h}-\phi_{S}\right)} / F_{U U}$ as a function of $x$ are displayed in figure 15 in addition to preliminary COMPASS data from ref. [167]. The asymmetry is very small, so at the current stage one may conclude that the WW-type 


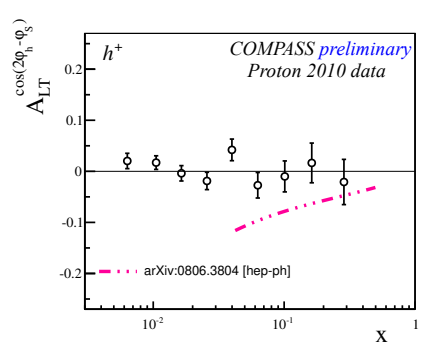

(a)

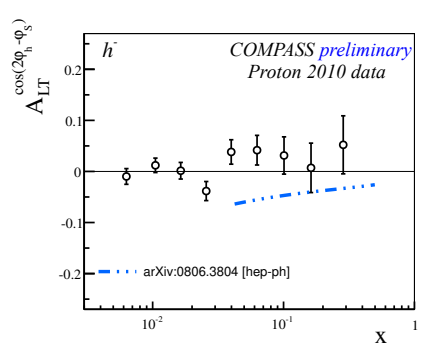

(b)

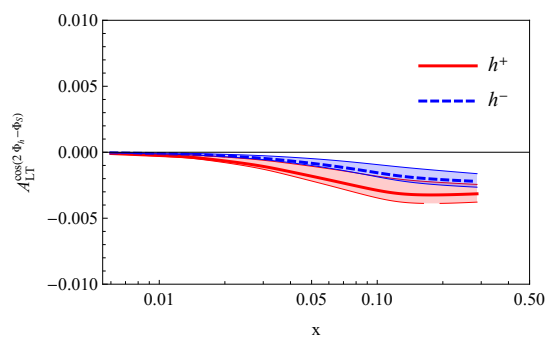

(c)

Figure 15. $A_{L T}^{\cos \left(2 \phi_{h}-\phi_{S}\right)}$ as a function of $x$ from a proton target: preliminary COMPASS data [167] $(\mathrm{a}, \mathrm{b})$, and our calculation for COMPASS kinematics (c) shown separately for reasons explained in the caption of figure 9 .

approximation for the asymmetry $A_{L T}^{\cos \left(2 \phi_{h}-\phi_{S}\right)}$ is compatible with available data. In view of the smallness of the effect (cf. figure 15), it might be difficult to obtain more quantitative insights in the near future.

For completeness, figure 15 shows also results from a quark-diquark model calculation, where a more sizable asymmetry was predicted [170]. Let us remark that the $A_{L T}^{\cos \left(2 \phi_{h}-\phi_{S}\right)}$ asymmetry was also studied in a different spectator model in ref. [199] predicting asymmetries of the same sign but of smaller magnitudes of $\mathcal{O}(1 \%)$.

\subsection{Subleading-twist $A_{L L}^{\cos \phi_{h}}$}

In WW-type approximation the only contribution to $F_{L L}^{\cos \phi_{h}}$ is due to $g_{L}^{\perp q}\left(x, k_{\perp}^{2}\right)$, which we assume to have a Gaussian $k_{\perp}$-behavior according to (B.9e) in appendix B.3 with the Gaussian width $\left\langle k_{\perp}^{2}\right\rangle_{g_{L}^{\perp}}=\left\langle k_{\perp}^{2}\right\rangle_{g_{1}}$. The structure function $F_{L L}^{\cos \phi_{h}}$ reads

$$
\begin{aligned}
F_{L L}^{\cos \phi_{h}}\left(x, z, P_{h T}\right) & =-\frac{2 M_{N}}{Q} x \sum_{q} e_{q}^{2} x g_{L}^{\perp(1) q}(x) D_{1}^{q}(z) b_{\mathrm{B}}^{(1)}\left(\frac{z P_{h T}}{\lambda}\right) \mathcal{G}\left(P_{h T}\right), \\
F_{L L}^{\cos \phi_{h}}\left(x, z,\left\langle P_{h T}\right\rangle\right) & =-\frac{2 M_{N}}{Q} x \sum_{q} e_{q}^{2} x g_{L}^{\perp(1) q}(x) D_{1}^{q}(z) c_{\mathrm{B}}^{(1)}\left(\frac{z}{\lambda^{1 / 2}}\right),
\end{aligned}
$$

where $\lambda=z^{2}\left\langle k_{\perp}^{2}\right\rangle_{g_{L}^{\perp}}+\left\langle P_{\perp}^{2}\right\rangle_{D_{1}}, b_{\mathrm{B}}^{(1)}=2 M_{N}, c_{\mathrm{B}}^{(1)}=\sqrt{\pi} M_{N}$, see appendix B.5. Finally we explore the WW-type approximation (3.3c) to relate $x g_{L}^{\perp(1) a}(x)=\frac{\left\langle k_{\perp}^{2}\right\rangle_{g_{1}}}{2 M_{N}^{2}} g_{1}^{a}(x)$.

The asymmetry $A_{L L}^{\cos \phi_{h}}=F_{L L}^{\cos \phi_{h}} / F_{U U}$ predicted by the WW-type approximation in this case is compatible with preliminary COMPASS [176, 180] (see figure 16) and HERMES [127] data. We remark that previously this asymmetry was studied in basically WW-type approximation in [201] and more recently also in a model study [202]. The predictions from both works are included in figure 16 for comparison.

\subsection{Subleading-twist $A_{U L}^{\sin \phi_{h}}$}

This was historically the first measurement of a single-spin asymmetry in SIDIS, by HERMES [179, 203], and consequently subject to numerous phenomenological and model studies [204-211]. A more recent model study was reported in [212]. 


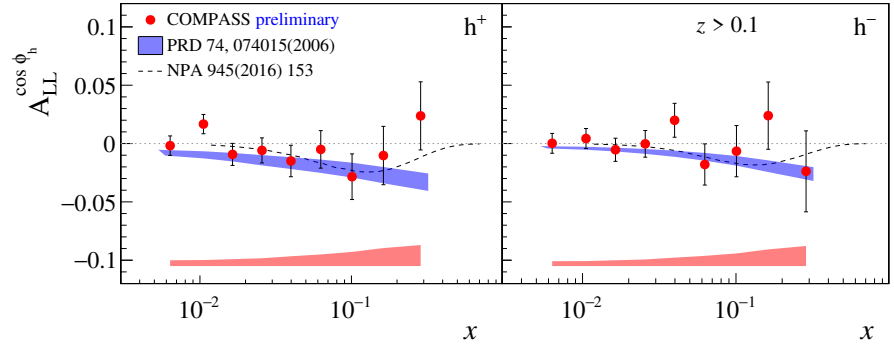

(a)

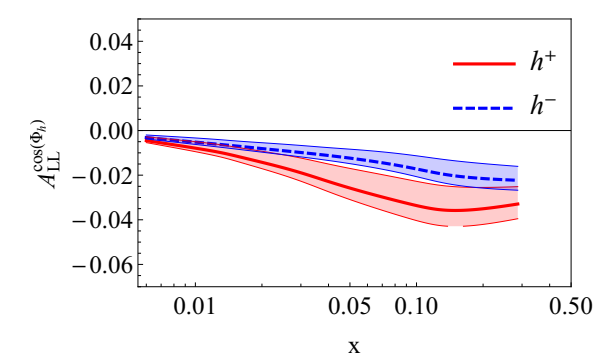

(c)

Figure 16. $A_{L L}^{\cos \left(\phi_{h}\right)}$ as a function of $x$ : preliminary COMPASS data $[176,180](\mathrm{a}, \mathrm{b})$, and our calculation for COMPASS kinematics in WW-type approximation (c) shown separately for reasons explained in the caption of figure 9 .

In WW-type approximation $A_{U L}^{\sin \phi_{h}}$ is described by $h_{L}^{q}\left(x, k_{\perp}^{2}\right)$, for which we assume the Gaussian Ansatz (B.9f) in appendix B.3 with $\left\langle k_{\perp}^{2}\right\rangle_{h_{L}}=\left\langle k_{\perp}^{2}\right\rangle_{h_{1}}$. We explore (3.3f) to estimate $x h_{L}^{q}(x)=-2 h_{1 L}^{\perp(1) q}(x)$ and express $h_{1 L}^{\perp(1) q}(x)$ through $h_{1}^{a}(x)$ according to (3.6b). This yields

$$
\begin{aligned}
F_{U L}^{\sin \phi_{h}}\left(x, z, P_{h T}\right) & =\frac{2 M_{N}}{Q} x \sum_{q} e_{q}^{2} x h_{L}^{q}(x) H_{1}^{\perp(1) q}(z) b_{\mathrm{A}}^{(1)}\left(\frac{z P_{h T}}{\lambda}\right) \mathcal{G}\left(P_{h T}\right), \\
F_{U L}^{\sin \phi_{h}}\left(x, z,\left\langle P_{h T}\right\rangle\right) & =\frac{2 M_{N}}{Q} x \sum_{q} e_{q}^{2} x h_{L}^{q}(x) H_{1}^{\perp(1) q}(z) c_{\mathrm{A}}^{(1)}\left(\frac{z}{\lambda^{1 / 2}}\right)
\end{aligned}
$$

where $\lambda=z^{2}\left\langle k_{\perp}^{2}\right\rangle_{h_{L}}+\left\langle P_{\perp}^{2}\right\rangle_{H_{1}^{\perp}}$ and $b_{\mathrm{A}}^{(1)}=2 m_{h}$ and $c_{\mathrm{A}}^{(1)}=\sqrt{\pi} m_{h}$, see appendix B.5 for details.

The asymmetries $A_{U L}^{\sin \phi_{h}}=F_{U L}^{\sin \phi_{h}} / F_{U U}$ are compared to HERMES, JLab, and preliminary COMPASS data in figure 17. The WW-type approximation seems not incompatible with data for negative pions and hadrons, but underestimates the magnitude of the asymmetry for positive pions and hadrons at HERMES and COMPASS. For neutral pions the approximation predicts a negligibly small effect (due to cancelling contributions from favored und unfavored Collins fragmentation function) and is not able to explain the large effect observed at HERMES and JLab for $\pi^{0}$ in the large- $x$ region $0.1<x<0.5$ in figure 17. This indicates that in this asymmetry the tilde-terms are not negligible, and have a characteristic flavor dependence that is distinct from that of the Collins effect.

\subsection{Subleading-twist $A_{U T}^{\sin \phi_{S}}$}

In this structure function some interesting new features occur. The first feature is that after applying the WW-type approximation, not one but three terms are left, cf. eqs. (2.18e), (4.2c): two terms proportional to $h_{T}^{\perp q}\left(x, k_{\perp}^{2}\right)$ and $h_{T}^{q}\left(x, k_{\perp}^{2}\right)$, respectively, and one term proportional to $f_{T}^{q}\left(x, k_{\perp}^{2}\right)$, which is associated with the second interesting feature. This Todd TMD must satisfy the sum rule $\int d^{2} k_{\perp} f_{T}^{q}\left(x, k_{\perp}^{2}\right)=0$, see (2.13) and section 3.8. This could be implemented in two ways. One could describe it with a superposition of Gaussians, see appendix B.4. But at this point we have no guidance from phenomenology or theory 


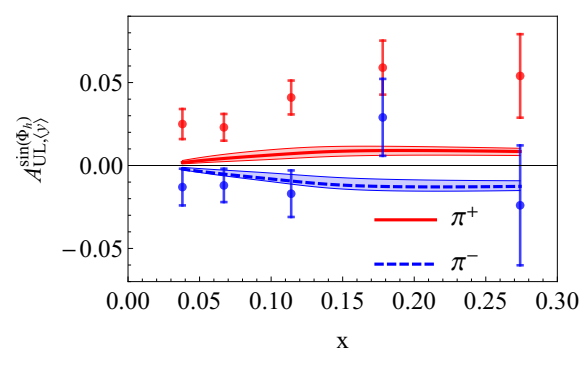

(a)

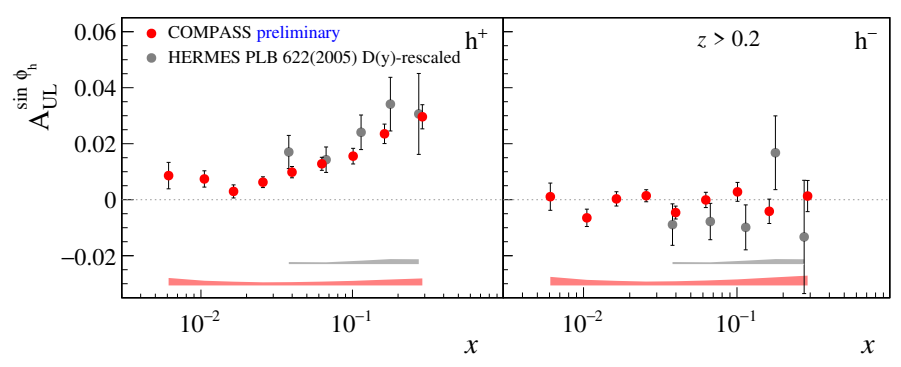

(c)

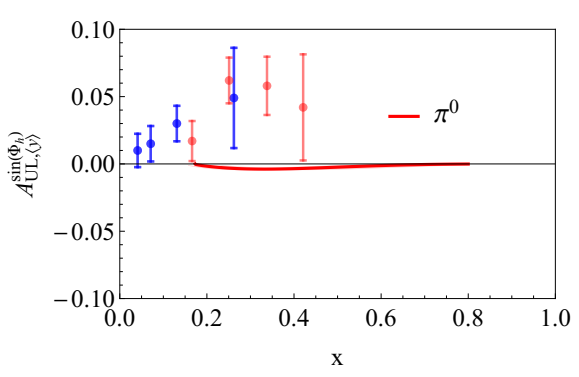

(b)

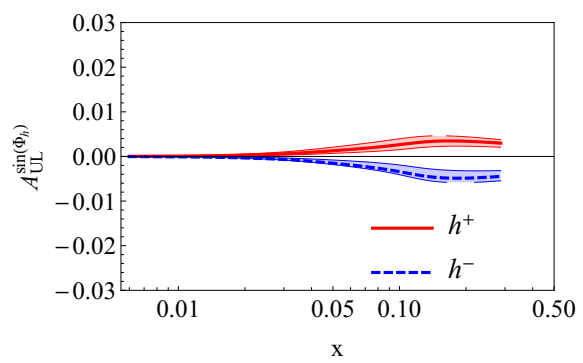

(e)

Figure 17. $A_{U L}^{\sin \phi_{h}}$ for proton vs. $x$ from WW-type approximation and comparison to the data. $\pi^{ \pm}$from HERMES [213] (a), $\pi^{0}$ from HERMES (blue) [203] and JLab (red) [181] (b), preliminary COMPASS data $[176,180](\mathrm{c}, \mathrm{d})$, and our calculation for COMPASS kinematics in WW-type approximation (e) shown separately for reasons explained in the caption of figure 9 .

to fix additional parameters. So we choose an alternative and pragmatic solution, namely to neglect the contribution of $f_{T}^{q}\left(x, k_{\perp}^{2}\right){ }^{8}$ Assuming Gaussian Ansatz (B.9g), (B.9h) for $h_{T}^{\perp q}\left(x, k_{\perp}^{2}\right), h_{T}^{q}\left(x, k_{\perp}^{2}\right)$ in appendix B.3 and relating them to transversity via the WW-type approximations $(3.3 \mathrm{~g}),(3.3 \mathrm{~h})$, the expression for $F_{U T}^{\sin \phi_{S}}$ is given in terms of a single term

$$
\begin{aligned}
F_{U T}^{\sin \phi_{S}}\left(x, z, P_{h T}\right) & =\frac{2 M_{N}}{Q} x \sum_{q} e_{q}^{2} h_{1}^{(1) q}(x) H_{1}^{\perp(1) q}(z) \frac{4 z^{2} m_{h} M_{N}}{\lambda}\left(1-\frac{P_{h T}^{2}}{\lambda}\right) \mathcal{G}\left(P_{h T}\right) \\
F_{U T}^{\sin \phi_{S}}(x, z) & =0 .
\end{aligned}
$$

with $\lambda=z^{2}\left\langle k_{\perp}^{2}\right\rangle_{h_{T}^{\perp}}+\left\langle P_{\perp}^{2}\right\rangle_{H_{1}^{\perp}}$ and $\left\langle k_{\perp}^{2}\right\rangle_{h_{T}^{\perp}}=\left\langle k_{\perp}^{2}\right\rangle_{h_{T}}=\left\langle k_{\perp}^{2}\right\rangle_{h_{1}}$. The third interesting feature is the occurrence of a term that drops out upon integrating the structure function over $P_{h T}$, cf. (7.7a) vs. (7.7b). This is a property of the weight $\omega_{\mathrm{B}}^{\{2\}}$, see $(2.19)$ and appendix B (which appears also in $F_{L T}^{\cos \phi_{S}},(2.18 \mathrm{f})$, where it drops out in WW-type approximation). This property can help to discriminate experimentally the terms associated with this weight.

The final result in $(7.7 \mathrm{~b})$ is the consistent result for $F_{U T}^{\sin \phi_{S}}(x, z)$ in WW-type approximation, see (2.20d). Our prediction is therefore $A_{U T}^{\sin \phi_{S}}(x)=0$. Preliminary COMPASS [167] (figure 18 top) and HERMES [168] (figure 18 bottom) data indicate that at

\footnotetext{
8 This corresponds to using a "single Gaussian" as $f_{T}^{q}\left(x, k_{\perp}^{2}\right)=f_{T}^{q}(x) \frac{\exp \left(-k_{\perp}^{2} /\left\langle k_{\perp}^{2}\right\rangle_{f_{T}}\right)}{\pi\left\langle k_{\perp}^{2}\right\rangle_{f_{T}}}$ with the "coefficient" $f_{T}^{q}(x)=0$ as dictated by the sum rule (2.13).
} 


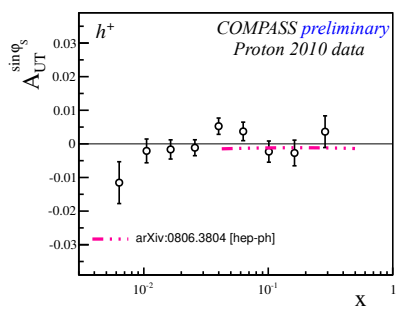

(a)

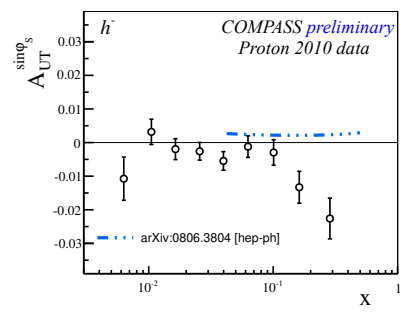

(b)

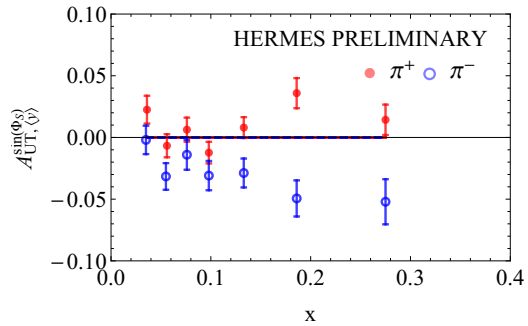

(c)

Figure 18. Subleading-twist asymmetry $A_{U T}^{\sin \phi_{S}}(x)$, which is predicted to vanish in the WW-type approximation: preliminary COMPASS [167] (a,b), and HERMES data [168] (c).

$x \gtrsim 0.1$ the signal is clearly non-zero and thus inconsistent with this WW-type prediction. In order to describe the data, it is therefore necessary to explicitly model the involved tilde-functions. For a recent model study we refer to ref. [214].

\subsection{Subleading-twist $A_{U T}^{\sin \left(2 \phi_{h}-\phi_{S}\right)}$}

In this asymmetry, two terms survive the WW-type approximation. Using the Gaussian Ansatz for $f_{T}^{\perp q}\left(x, k_{\perp}^{2}\right), h_{T}^{\perp q}\left(x, k_{\perp}^{2}\right), h_{T}^{q}\left(x, k_{\perp}^{2}\right)$, according to (B.9g), (B.9h), (B.9i) in appendix B.3, with $\left\langle k_{\perp}^{2}\right\rangle_{h_{T}^{\perp}}=\left\langle k_{\perp}^{2}\right\rangle_{h_{T}}=\left\langle k_{\perp}^{2}\right\rangle_{h_{1 T}^{\perp}}$ and $\left\langle k_{\perp}^{2}\right\rangle_{f_{T}^{\perp}}=\left\langle k_{\perp}^{2}\right\rangle_{f_{1 T}^{\perp}}$ yields the expressions

$$
\begin{aligned}
F_{U T}^{\sin \left(2 \phi_{h}-\phi_{S}\right)}\left(x, z, P_{h T}\right)= & \frac{2 M_{N}}{Q} x \sum_{q} e_{q}^{2}\left[x f_{T}^{\perp(2) q}(x) D_{1}^{q}(z) b_{\mathrm{C}}^{(2)}\left(\frac{z P_{h T}}{\lambda}\right)^{2} \mathcal{G}\left(P_{h T}\right)\right. \\
& \left.+\frac{x}{2}\left(h_{T}^{(1) q}(x)+h_{T}^{\perp(1) q}(x)\right) H_{1}^{\perp(1) q}(z) b_{\mathrm{AB}}^{(2)}\left(\frac{z P_{h T}}{\lambda}\right)^{2} \mathcal{G}\left(P_{h T}\right)\right], \\
F_{U T}^{\sin \left(2 \phi_{h}-\phi_{S}\right)}\left(x, z,\left\langle P_{h T}\right\rangle\right)= & \frac{2 M_{N}}{Q} x \sum_{q} e_{q}^{2}\left[x f_{T}^{\perp(2) q}(x) D_{1}^{q}(z) c_{\mathrm{C}}^{(2)}\left(\frac{z}{\lambda^{1 / 2}}\right)^{2}\right. \\
& \left.+\frac{x}{2}\left(h_{T}^{(1) q}(x)+h_{T}^{\perp(1) q}(x)\right) H_{1}^{\perp(1) q}(z) c_{\mathrm{AB}}^{(2)}\left(\frac{z}{\lambda^{1 / 2}}\right)^{2}\right]
\end{aligned}
$$

with respectively $\lambda=z^{2}\left\langle k_{\perp}^{2}\right\rangle_{f_{T}^{\perp}}+\left\langle P_{\perp}^{2}\right\rangle_{D_{1}}$ in the first, and $\lambda=z^{2}\left\langle k_{\perp}^{2}\right\rangle_{h_{T}^{\perp}}+\left\langle P_{\perp}^{2}\right\rangle_{H_{1}^{\perp}}$ in the second terms in (7.8a), (7.8b). The coefficients $b_{i}^{(2)}$ and $c_{i}^{(2)}$ are defined in appendix B.5. In the next step we explore the WW-type approximations $(3.3 \mathrm{~g}),(3.3 \mathrm{~h}),(3.4 \mathrm{f})$ to relate $x f_{T}^{\perp(2) q}(x)=\frac{\left\langle k_{\perp}^{2}\right\rangle_{f_{1 T}^{\perp}}}{M_{N}^{2}} f_{1 T}^{\perp(1) q}(x)$ and $-\frac{1}{2} x\left(h_{T}^{(1) q}(x)+h_{T}^{\perp(1) q}(x)\right)=h_{1 T}^{\perp(2) q}(x)$.

The asymmetries $A_{U T}^{\sin \left(2 \phi_{h}-\phi_{S}\right)}=F_{U T}^{\sin \left(2 \phi_{h}-\phi_{S}\right)} / F_{U U}$ are plotted in figure 19 in comparison to preliminary COMPASS [167] and HERMES [168] data. The predicted asymmetry is small and compatible with the data that are consistent with a zero effect within uncertainties. For comparison, figure 15 shows also the predictions from the quark-diquark model of ref. [170]. More recently the $A_{U T}^{\sin \left(2 \phi_{h}-\phi_{S}\right)}$ was also studied in the model approach of ref. [214]. 


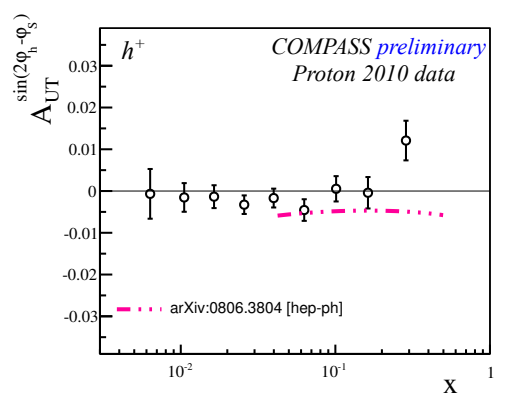

(a)

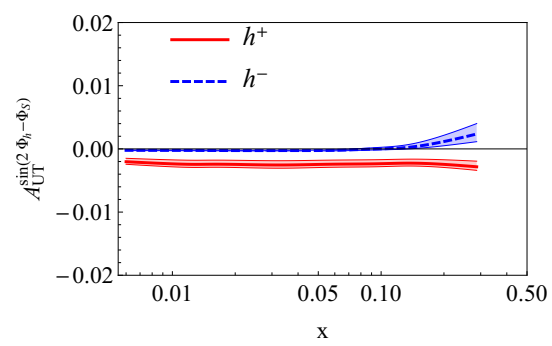

(c)

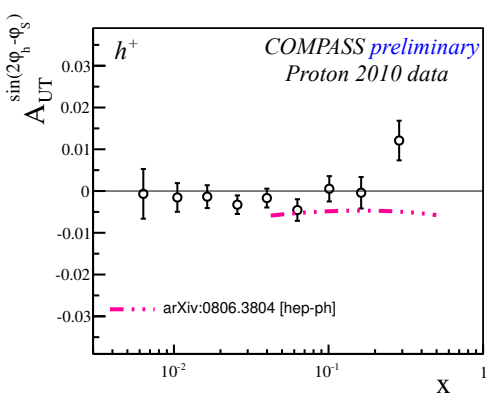

(b)

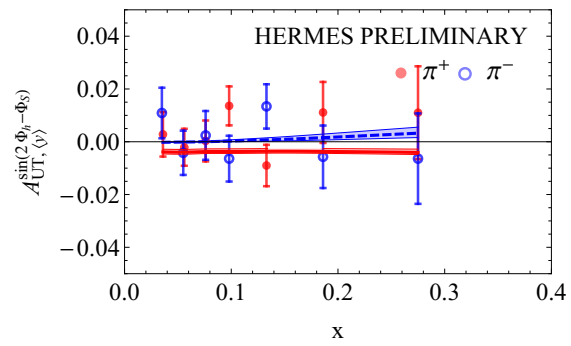

(d)

Figure 19. $A_{U T}^{\sin \left(2 \phi_{h}-\phi_{S}\right)}(x)$ : preliminary COMPASS data [167] (a,b) and our calculation for COMPASS kinematics in WW-type approximation (c) shown separately for reasons explained in the caption of figure 9. Similarly $A_{U T,\langle y\rangle}^{\sin \left(2 \phi_{h}-\phi_{S}\right)}(x)$ vs preliminary HERMES data [168] (d).

\subsection{Subleading-twist $A_{U U}^{\cos \phi_{h}}$}

Historically this was the earliest azimuthal SIDIS asymmetry to be discussed in literature: the first prediction for this asymmetry from intrinsic $k_{\perp}$ was made in [160, 215], a first measurement was reported in [216]. ${ }^{9}$ This structure function contains after the WW-type approximation initially two contributions, proportional to $f^{\perp}\left(x, k_{\perp}^{2}\right)$ and $h^{q}\left(x, k_{\perp}^{2}\right)$. The latter is T-odd and obeys the sum rule (2.13). We treat $h^{q}\left(x, k_{\perp}^{2}\right)$ exactly as $f_{T}^{q}\left(x, k_{\perp}^{2}\right)$ in section 7.6. Using the Gaussian Ansatz for $f^{\perp}\left(x, k_{\perp}^{2}\right)$ in (B.9j) of appendix B.3, we obtain

$$
\begin{aligned}
F_{U U}^{\cos \phi_{h}}\left(x, z, P_{h T}\right) & =\frac{2 M_{N}}{Q} x \sum_{q} e_{q}^{2}\left[-x f^{\perp(1) q}(x) D_{1}^{q}(z) b_{\mathrm{B}}^{(1)}\left(\frac{z P_{h T}}{\lambda}\right) \mathcal{G}\left(P_{h T}\right)\right], \\
F_{U U}^{\cos \phi_{h}}\left(x, z,\left\langle P_{h T}\right\rangle\right) & =\frac{2 M_{N}}{Q} x \sum_{q} e_{q}^{2}\left[-x f^{\perp(1) q}(x) D_{1}^{q}(z) c_{\mathrm{B}}^{(1)}\left(\frac{z}{\lambda^{1 / 2}}\right)\right],
\end{aligned}
$$

with $\lambda=z^{2}\left\langle k_{\perp}^{2}\right\rangle_{f^{\perp}}+\left\langle P_{\perp}^{2}\right\rangle_{D_{1}}$. The coefficients $b_{i}^{(1)}$ and $c_{i}^{(1)}$ are defined in appendix B.5. Note that $(7.9 \mathrm{a})$ is valid in the "scheme" of footnote 8 , but (7.9a) holds independent of how one implements the sum rule (2.13) (as in footnote 8 or appendix B.4).

\footnotetext{
${ }^{9}$ First hints [217] of azimuthal modulations in SIDIS date back to the early 1970s, i.e., 10 years before the CERN measurements, but (unfortunately) were discarded by the authors.
} 

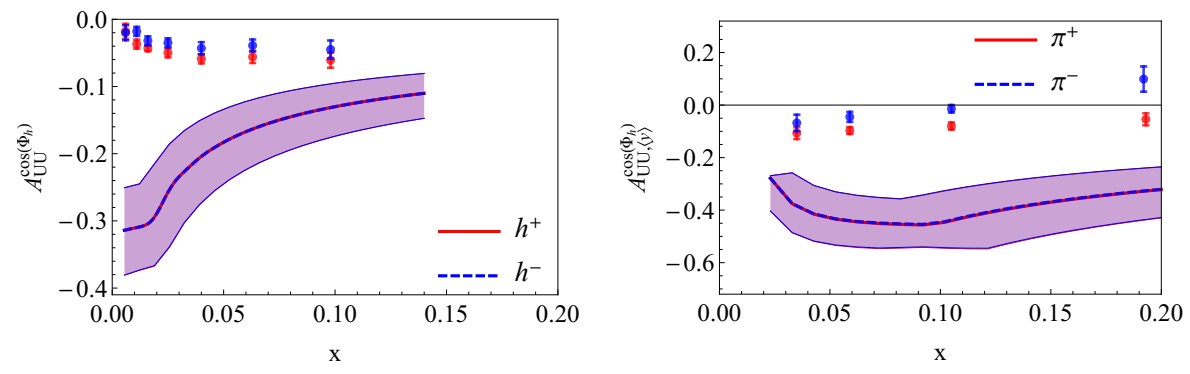

Figure 20. Left panel: asymmetry $A_{U U}^{\cos \phi_{h}}$ for positive and negative hadrons at COMPASS for a proton target [159]. Right panel: the corresponding $A_{U U,\langle y\rangle}^{\cos \phi_{h}}$ for $\pi^{ \pm}$from HERMES [158].

For $f^{\perp(1)}(x)$ we explore (3.3b) as $x f^{\perp(1) q}(x)=\frac{\left\langle k_{\perp}^{2}\right\rangle_{f_{1}}}{2 M_{N}^{2}} f_{1}^{q}(x)$ and assume for its Gaussian width $\left\langle k_{\perp}^{2}\right\rangle_{f^{\perp}}=\left\langle k_{\perp}^{2}\right\rangle_{f_{1}}$. The latter means the Gaussian factors of $F_{U U}^{\cos \phi_{h}}$ and $F_{U U}$ cancel out, i.e. at some point for $P_{h T} \gtrsim 1 \mathrm{GeV}$ we would obtain from (7.9a) an asymmetry $A_{U U}^{\cos \phi_{h}}=F_{U U}^{\cos \phi_{h}} / F_{U U}$ exceeding $100 \%$ and violating unitarity. This is of course an artifact of our approximations, and reminds us that Gaussian and WW-type approximations as well as the entire TMD formalism must be applied to small $P_{h T} \ll Q$.

The asymmetries $A_{U U}^{\cos \phi_{h}}$ were measured by EMC [216], at JLab [122, 218], HERMES [158], and COMPASS [159]. In figure 20 we compare our predictions to the HERMES and COMPASS data. The WW-type approximation tends to overestimate the data at COMPASS especially in the small- $x$ region. It is also not compatible with the flavor dependence seen at HERMES. However, both experiments seem not to agree for instance on the shape of the asymmetry for negative pions or hadrons. More experimental and theoretical work is needed to clarify whether this could be due to power corrections.

\section{Conclusions}

In this work a comprehensive and complete treatment of SIDIS spin and azimuthal asymmetries was presented. The theoretical and phenomenological understanding of most of the leading-twist SIDIS structure functions for the production of unpolarized hadrons is relatively advanced: factorization is proven, and each structure functions is unambiguously expressed in terms of one of eight twist-2 TMDs convoluted with one of two twist-2 FFs. For subleading-twist SIDIS structure functions the situation is far more complex for two reasons. First, factorization is not proven and must be assumed. Second, each of the subleading-twist structure functions receives four or six contributions from various TMDs and FFs one of which is twist-2 and the other twist-3. Clearly, to make predictions for new experiments or interpret early data, an organizing theoretical guideline is needed.

In this work we have explored the so-called WW-type approximation as a candidate guideline for the description of SIDIS structure functions. This approximation consists of a systematic neglect of $\bar{q} g q$-terms in the correlators defining the TMDs and FFs. We have shown that in such an approach all twist-2 and twist-3 structure functions can be described in terms of eight basis functions: six TMDs and two FFs, which are each twist-2. All other TMDs and FFs, assuming this approximation, are either related to the basis functions or 
vanish. We remark that the generalized parton model approach of ref. [219] provides a description that is largely equivalent to ours.

To make this work self-contained, we included a review of the available phenomenological information on the basis functions, which is given in terms of six SIDIS structure functions. (Of course, one cannot extract eight basis functions from six observables: the extraction of two basis functions, the unpolarized TMD and FF $f_{1}^{q}\left(x, k_{\perp}^{2}\right)$ and $D_{1}^{q}\left(x, P_{\perp}^{2}\right)$, makes also use of other experiments, most notably Drell-Yan and hadron production in $e^{+} e^{-}$annihilations.)

The WW-type approximation for TMDs and FFs is inspired by the observation that the classical WW-approximation for the twist-3 DIS structure function $g_{2}(x)$ (or PDF $\left.g_{T}^{q}(x)\right)$ works well. This was predicted in theoretical studies in the instanton model of the QCD vacuum, and confirmed by data and lattice QCD studies. The instanton vacuum model predicts an analogous WW approximation for the chirally odd twist-3 PDF $h_{L}^{q}(x)$ to work similarly well. This prediction remains to be tested in experiment.

In each case, $g_{T}^{q}(x)$ and $h_{L}^{q}(x)$, we deal with nucleon matrix elements of different $\bar{q} g q$ correlators, which are assumed to be small. Can one generalize these approximations to TMDs? This is a key question, which has been addressed in the past in literature in selected cases. This work is the first systematic investigation of this question. As in the unintegrated correlators one deals with different classes of operators, we prefer to speak of the WW-type approximation to distinguish from the collinear case.

We have studied in detail all SIDIS structure function in this approximation. This includes a review of results from lattice QCD calculations, effective theories and models. We found that from theoretical point of view the WW-type approximations receive certain support, though there is less evidence than in the collinear case. Most importantly, we have conducted systematic tests of WW approximations with available published or preliminary (and soon to be published) SIDIS data from HERMES, COMPASS, and JLab.

We found the following results. The two leading-twist structure functions amenable to WW-type approximations, $F_{L T}^{\cos \left(\phi_{h}-\phi_{S}\right)}$ and $F_{U L}^{\sin \left(2 \phi_{h}\right)}$, are well-described (the former) or at least compatible (the latter) with the data in this approximation. For $F_{U L}^{\sin \left(2 \phi_{h}\right)}$ more precise data are needed, but also in this case the trend is encouraging especially thanks to the recent preliminary COMPASS data. We have also shown that our approach satisfies positivity inequalities, which is a non-trivial consistency check considering the crude approximations (WW-type, Gaussian Ansatz for TMDs) in our approach.

At subleading twist the WW-type approximation for the structure functions $F_{L T}^{\cos \phi_{S}}$, $F_{L T}^{\cos \left(2 \phi_{h}-\phi_{S}\right)}, F_{L L}^{\cos \phi_{h}}, F_{U T}^{\sin \left(2 \phi_{h}-\phi_{S}\right)}$ is compatible with data, too. Some of these asymmetries are predicted to be very small in the WW-type approximation, sometimes smaller than a fraction of a percent. This is compatible with the available data in the sense that the data are consistent with zero within their statistical accuracy. This cannot be considered a thorough evidence for the applicability of the WW-type approximations, but on the positive side we also observe no hints that the WW-type approximations fail in these cases.

In the case of the subleading-twist structure functions $F_{U U}^{\cos \phi_{h}}, F_{U L}^{\sin \phi_{h}}, F_{L U}^{\sin \phi_{h}}$, and $F_{U T}^{\sin \phi_{S}}$ the situation is clearer and indicates that here the WW-type approximations do not work. Incidentally, these asymmetries include the very first non-zero azimuthal asym- 
metry measured in unpolarized SIDIS $\left(F_{U U}^{\cos \phi_{h}}\right)$, the very first non-zero target single-spin asymmetry measured in SIDIS $\left(F_{U L}^{\sin \phi_{h}}\right)$, and the beam-spin asymmetry $\left(F_{L U}^{\sin \phi_{h}}\right)$. The WW-type prediction for $F_{U U}^{\cos \left(\phi_{h}\right)}$ tends to overshoot the data. In the case of $F_{U L}^{\sin \phi_{h}}$ the WW-type approximation undershoots data by a factor of two or so. Most interestingly, in the case of $F_{L U}^{\sin \phi_{h}}$ the WW-type approximation predicts exactly a zero asymmetry, but experiments see small but non-zero effect.

The non-applicability of the WW-type approximation in these cases should not be too surprising. After all it is a crude method to model TMDs and FFs and an uncontrollable "expansion" (in nuclear physics the concept of 2-body, 3-body, etc operators is well-justified and an effective expansion can be conducted; in the case of TMDs, however, it is less appropriate to speak about a systematic expansion in terms of $\bar{q} q, \bar{q} q q$, etc correlators). It will be very interesting to learn whether, e.g., in $F_{U L}^{\sin \phi_{h}}$ or $F_{L U}^{\sin \phi_{h}}$ a single $\bar{q} g q$-term is anomalously large, or whether it is an accumulative effect of several small terms $\bar{q} g q$-terms adding up to the observed asymmetry.

Among all SIDIS structure functions $F_{L U}^{\mathrm{sin} \phi_{h}}$ emerges as a particularly interesting case: this asymmetry is due to $\bar{q} g q$ only, without "contamination" from $\bar{q} q$ terms. Thus $F_{L U}^{\sin \phi_{h}}$ offers a unique view on the physics of $\bar{q} g q$ correlators, worth exploring for its own sake.

The results presented in this work are of importance for several reasons. To the best of our knowledge, it is the first complete study of all SIDIS structure functions up to twist-3 using systematically a common theoretical guideline. The results are useful for experiments prepared in the near term (JLab 12) or proposed in the long term (Electron-Ion Collider), and provide helpful input for Monte Carlo event generators [33]. Our predictions will help to pave the way towards a better understanding of the quark-gluon structure of the nucleon beyond leading twist.

\section{Acknowledgments}

First and foremost, the authors would like to thank their families for patience and constant support throughout writing this paper. The authors would like to thank Werner Vogelsang for many useful discussions. This work was partially supported by the U.S. Department of Energy under Contract No. DE-AC05-06OR23177 (A.P.) and within the TMD Collaboration framework, and by the National Science Foundation under Contract No. PHY-1623454 (A.P.) and Contract No. PHY-1812423 (P.S.), and the Wilhelm Schuler Stiftung (P.S.). The work was supported by DOE grant no. DE-FG02-04ER41309. We thank the COMPASS and HERMES Collaborations for the permissions to show their preliminary data on several figures.

\section{A The "minimal basis" of TMDs and FFs}

This appendix describes the technical details of the parametrizations used in this work.

\section{A.1 Unpolarized functions $f_{1}^{a}\left(x, k_{\perp}^{2}\right)$ and $D_{1}^{a}\left(z, P_{\perp}^{2}\right)$}

In this work we use the leading-order parametrizations from [118] for the unpolarized PDF $f_{1}^{a}(x)$ and from [119] for the unpolarized FF $D_{1}^{a}(z)$. If not otherwise stated the 


\begin{tabular}{|ccccc|}
\hline study & $\begin{array}{c}\left\langle Q^{2}\right\rangle,\langle x\rangle,\langle z\rangle \\
{\left[\mathrm{GeV}^{2}\right]}\end{array}$ & $\begin{array}{c}\left\langle k_{\perp}^{2}\right\rangle_{f_{1}} \\
{\left[\mathrm{GeV}^{2}\right]}\end{array}$ & $\begin{array}{c}\left\langle P_{\perp}^{2}\right\rangle_{D_{1}} \\
{\left[\mathrm{GeV}^{2}\right]}\end{array}$ & $\begin{array}{c}\left\langle k_{\perp}^{2}\right\rangle_{g_{1}} \\
{\left[\mathrm{GeV}^{2}\right]}\end{array}$ \\
\hline fit of [110] & $5.0,0.1,0.3$ & $\sim 0.25$ & $\sim 0.2$ & - \\
fit of [113] & $2.5,0.1,0.4$ & $0.38 \pm 0.06$ & $0.16 \pm 0.01$ & - \\
fit of [115] & $2.4,0.1,0.3$ & $0.57 \pm 0.08$ & $0.12 \pm 0.01$ & - \\
fit of [114] & $2.4,0.1,0.5$ & $\sim 0.3$ & $\sim 0.18$ & - \\
lattice [67] & $4.0,-,-$ & $0.14-0.15$ & - & $0.11-0.15$ \\
\hline
\end{tabular}

Table 1. Gaussian model parameters for $f_{1}^{a}\left(x, k_{\perp}^{2}\right), D_{1}^{a}\left(z, P_{\perp}^{2}\right), g_{1}^{a}\left(x, k_{\perp}^{2}\right)$ from phenomenological and lattice QCD studies. The kinematics to which the phenomenological results and the renormalization scale of the lattice results are indicated. The range of lattice values indicates flavor dependence (first number refers to $u$-flavor, second number to $d$-flavor).

parametrizations are taken at the scale $Q^{2}=2.4 \mathrm{GeV}^{2}$ typical for many currently available SIDIS data. These parametrizations were used in [110] and other works whose extractions we adopt.

To describe the transverse momentum dependence of $f_{1}^{a}\left(x, k_{\perp}^{2}\right)$ and $D_{1}^{a}\left(z, P_{\perp}^{2}\right)$ we use the Gaussian Ansatz in eqs. (4.5a), (4.5b). All early [110-113] and some recent [114] analyses employed flavor and $x$ - or $z$-independent widths $\left\langle k_{\perp}^{2}\right\rangle$ and $\left\langle P_{\perp}^{2}\right\rangle$. In the analysis [115] of HERMES multiplicities flavor-independence of the widths was assumed. On long run one may anticipate that new precision data will require to relax these assumptions. However, one may also expect that the Gaussian Ansatz will remain a useful approximation as long as one is interested in describing data on transverse hadron momenta $P_{h T} \ll Q$.

The parameters resulting from calculations or extractions are presented in table 1 . As most extractions of TMDs that we will use are done with the choice of $\left\langle k_{\perp}^{2}\right\rangle_{f_{1}}=0.25 \mathrm{GeV}^{2}$, $\left\langle P_{\perp}^{2}\right\rangle_{D_{1}}=0.2 \mathrm{GeV}^{2}$, for our numerical estimates in this work we will use these fixed widths.

Some comments are in order. In [110] no attempt was made to assign an uncertainty of the best fit result. The uncertainty of the numbers from [113] includes only the statistical uncertainty, but no systematic uncertainty. For comparison lattice results from [67] are included whose range indicates flavor-dependence (first number $u$-flavor, second number $d$-flavor). Notice that this is the contribution of the flavor averaged over contributions from the respective quarks and antiquarks. Chiral theories predict significant differences in the $k_{\perp}$-behavior of sea and valence quarks [120]. We will comment more on the lattice results in the next section. In view of the large (and partly underestimated) uncertainties and the fact that those parameters are anti-correlated the numbers from the different approaches quoted in table 1 can be considered to be in good agreement.

\section{A.2 Helicity distribution $g_{1}^{a}\left(x, k_{\perp}^{2}\right)$}

For the helicity $\mathrm{PDF} g_{1}^{a}(x)=\int d^{2} k_{\perp} g_{1}^{a}\left(x, k_{\perp}^{2}\right) \equiv \int d^{2} k_{\perp} g_{1 L}^{a}\left(x, k_{\perp}^{2}\right)$ we use in this work the leading-order parametrizations from [62]. If not otherwise stated the parametrizations are taken at the scale $Q^{2}=2.4 \mathrm{GeV}^{2}$. 
In lack of phenomenological information on the $k_{\perp}$-dependence of $g_{1}^{a}\left(x, k_{\perp}^{2}\right)$ we explore lattice QCD results from [67] to constrain the Gaussian width in eq. (4.5c). On a lattice with pion and nucleon masses $m_{\pi} \approx 500 \mathrm{MeV}$ and $M_{N}=1.291(23) \mathrm{GeV}$ and with an axial coupling constant $g_{A}^{(3)}=1.209(36)$ reasonably close to its physical value $1.2695(29)$ the following results were obtained for the mean square transverse parton momenta [67]. For the unpolarized TMDs it was found $\left\langle k_{\perp}^{2}\right\rangle_{1}^{u}=(0.3741 \mathrm{GeV})^{2}$ and $\left\langle k_{\perp}^{2}\right\rangle_{f_{1}^{d}}=(0.3839 \mathrm{GeV})^{2}$. For the helicity TMDs it was found $\left\langle k_{\perp}^{2}\right\rangle_{g_{1}^{u}}=(0.327 \mathrm{GeV})^{2}$ and $\left\langle k_{\perp}^{2}\right\rangle_{g_{1}^{d}}=(0.385 \mathrm{GeV})^{2}$. These values are quoted in table 1.

The lattice values for $\left\langle k_{\perp}^{2}\right\rangle_{f_{1}}$ consistently underestimate the phenomenological numbers, see table 1. The exact reasons for that are unknown, but it is natural to think it might be related to the fact that the lattice predictions [67] do not refer to TMDs entering in SIDIS (or Drell-Yan or other process) because a different gauge link was chosen, see section 3.5. Still one may expect these results to bear considerable information on QCD dynamics. ${ }^{10}$ To make use of this information we shall assume that the lattice results [67] provide robust predictions for the ratios $\left\langle k_{\perp}^{2}\right\rangle_{g_{1}^{u}} /\left\langle k_{\perp}^{2}\right\rangle_{f_{1}^{u}} \approx 0.76$. With the phenomenological value $\left\langle k_{\perp}^{2}\right\rangle_{f_{1}}=0.25 \mathrm{GeV}^{2}$ we then obtain the estimate for the width of the helicity TMD $\left\langle k_{\perp}^{2}\right\rangle_{g_{1}}=0.19 \mathrm{GeV}^{2}$. In our phenomenological study we use this value for $u$-quarks and for simplicity also for $d$-quarks. Even though the lattice values indicate an interesting flavor dependence, see table 1 , for a proton target this is a very good approximation due to $u$ quark dominance. When precision data for deuterium and especially for ${ }^{3} \mathrm{He}$ from JLab become available, it will be interesting to re-investigate this point in detail.

\section{A.3 Sivers function $f_{1 T}^{\perp q}\left(x, k_{\perp}^{2}\right)$}

The Sivers distribution function was studied in refs. [44, 45, 134-136, 139, 219-222]. We will use parametrizations from refs. [134, 136, 219]:

$$
\begin{aligned}
\left\langle k_{\perp}^{2}\right\rangle_{f_{1 T}^{\perp}} & \equiv \frac{\left\langle k_{\perp}^{2}\right\rangle M_{1}^{2}}{\left\langle k_{\perp}^{2}\right\rangle+M_{1}^{2}}, \\
f_{1 T}^{\perp}\left(x, k_{\perp}^{2}\right) & =-\frac{M}{M_{1}} \sqrt{2 e} \mathcal{N}_{q}(x) f_{q / p}(x, Q) \frac{e^{-k_{\perp}^{2} /\left\langle k_{\perp}^{2}\right\rangle_{f_{1 T}^{\perp}}}}{\pi\left\langle k_{\perp}^{2}\right\rangle},
\end{aligned}
$$

where $M_{1}$ is a mass parameter, $M$ the proton mass and

$$
\mathcal{N}_{q}(x)=N_{q} x^{\alpha}(1-x)^{\beta} \frac{(\alpha+\beta)^{(\alpha+\beta)}}{\alpha^{\alpha} \beta^{\beta}} .
$$

The first moment of the Sivers function is:

$$
\begin{aligned}
f_{1 T}^{\perp(1) q}(x) & =-\frac{\sqrt{\frac{e}{2}}\left\langle k_{\perp}^{2}\right\rangle M_{1}^{3}}{M\left(\left\langle k_{\perp}^{2}\right\rangle+M_{1}^{2}\right)^{2}} \mathcal{N}_{q}(x) f_{q}(x, Q) \\
& =-\sqrt{\frac{e}{2}} \frac{1}{M M_{1}} \frac{\left\langle k_{\perp}^{2}\right\rangle_{f_{1 T}^{\perp}}^{2}}{\left\langle k_{\perp}^{2}\right\rangle} \mathcal{N}_{q}(x) f_{q}(x, Q)
\end{aligned}
$$

\footnotetext{
${ }^{10}$ The results [67] refer also to pion masses above the physical value. This caveat is presumably less critical and will be overcome as lattice QCD simulations are becoming feasible at physical pion masses.
} 


$$
\begin{array}{|llll}
N_{u}=0.40 & \alpha_{u}=0.35 & \beta_{u}=2.6 \\
N_{d}=-0.97 & \alpha_{d}=0.44 & \beta_{d}=0.90 & M_{1}^{2}=0.19\left(\mathrm{GeV}^{2}\right)
\end{array}
$$

Table 2. Best values of the fit of the Sivers functions. Table from ref. [136].

We can rewrite the parametrizations of the Sivers functions as

$$
f_{1 T}^{\perp q}\left(x, k_{\perp}^{2}\right)=f_{1 T}^{\perp(1) q}(x) \frac{2 M^{2}}{\pi\left\langle k_{\perp}^{2}\right\rangle_{f_{1 T}^{\perp}}^{2}} e^{-k_{\perp}^{2} /\left\langle k_{\perp}^{2}\right\rangle_{f_{1 T}^{\perp}}} .
$$

The fit the HERMES proton and COMPASS deuteron data from including only Sivers functions for $u$ and $d$ quarks was done in ref. [136], corresponding to seven free parameters, which are shown in table 2.

\section{A.4 Transversity $h_{1}^{q}\left(x, k_{\perp}^{2}\right)$ and Collins function $H_{1}^{\perp q}\left(x, P_{\perp}^{2}\right)$}

These functions were studied in refs. [49, 64, 65, 152-154]. The following shape was assumed for parametrizations in refs. [64, 65, 152]:

$$
\begin{aligned}
h_{1}^{q}\left(x, k_{\perp}^{2}\right) & =h_{1}^{q}(x) \frac{e^{-k_{\perp}^{2} /\left\langle k_{\perp}^{2}\right\rangle_{h_{1}}}}{\pi\left\langle k_{\perp}^{2}\right\rangle_{h_{1}}}, \\
h_{1}^{q}(x) & =\frac{1}{2} \mathcal{N}_{q}^{T}(x)\left[f_{1}(x)+g_{1}(x)\right], \\
H_{1 h / q}^{\perp}\left(z, P_{\perp}^{2}\right)=\frac{z m_{h}}{2 P_{\perp}} \Delta^{N} D_{h / q^{\uparrow}}\left(z, P_{\perp}^{2}\right) & =\frac{z m_{h}}{M_{C}} e^{-p_{\perp}^{2} / M_{C}^{2} \sqrt{2 e}} H_{1 h / q}^{\perp}(z) \frac{e^{-P_{\perp}^{2} /\left\langle P_{\perp}^{2}\right\rangle}}{\pi\left\langle P_{\perp}^{2}\right\rangle},
\end{aligned}
$$

with $m_{h}$ the mass of the produced hadron and

$$
\begin{aligned}
\mathcal{N}_{q}^{T}(x) & =N_{q}^{T} x^{\alpha}(1-x)^{\beta} \frac{(\alpha+\beta)^{(\alpha+\beta)}}{\alpha^{\alpha} \beta^{\beta}}, \\
H_{1 h / q}^{\perp}(z) & =\mathcal{N}_{q}^{C}(z) D_{h / q}(z) \\
\mathcal{N}_{q}^{C}(z) & =N_{q}^{C} z^{\gamma}(1-z)^{\delta} \frac{(\gamma+\delta)^{(\gamma+\delta)}}{\gamma^{\gamma} \delta^{\delta}}
\end{aligned}
$$

and $-1 \leq N_{q}^{T} \leq 1,-1 \leq N_{q}^{C} \leq 1$. The helicity distributions $g_{1}(x)$ are taken from ref. [223], parton distribution and fragmentation functions are the GRV98LO PDF set [62] and the DSS fragmentation function set [119]. Notice that with these choices both the transversity and the Collins function automatically obey their proper positivity bounds. Note that as in ref. [152] we use two Collins fragmentation functions, favored and disfavored ones, see ref. [152] for details on implementation, and corresponding parameters $N_{a}^{C}$ are then $N_{\text {fav }}^{C}$ and $N_{d i s}^{C}$. For numerical estimates we use parameters extracted in ref. [152], see table 3.

According to eq. (B.8) we obtain the following expression for the first moment of Collins fragmentation function:

$$
H_{1 h / q}^{\perp(1)}(z)=\frac{H_{1 h / q}^{\perp}(z) \sqrt{e / 2}\left\langle P_{\perp}^{2}\right\rangle M_{C}^{3}}{z m_{h}\left(M_{C}^{2}+\left\langle P_{\perp}^{2}\right\rangle\right)^{2}} .
$$




\begin{tabular}{|lll|}
\hline$N_{u}^{T}=0.46_{-0.14}^{+0.20}$ & $N_{d}^{T}=-1.00_{-0.00}^{+1.17}$ & \\
$\alpha=1.11_{-0.66}^{+0.89}$ & $\beta=3.64_{-3.37}^{+5.80}$ & $\left\langle k_{\perp}^{2}\right\rangle_{h_{1}}=0.25\left(\mathrm{GeV}^{2}\right)$ \\
\hline$N_{\text {fav }}^{C}=0.49_{-0.18}^{+0.20}$ & $N_{\text {dis }}^{C}=-1.00_{-0.00}^{+0.38}$ & \\
$\gamma=1.06_{-0.32}^{+0.45}$ & $\delta=0.07_{-0.07}^{+0.42}$ & $M_{C}^{2}=1.50_{-1.12}^{+2.00}\left(\mathrm{GeV}^{2}\right)$ \\
\hline
\end{tabular}

Table 3. Best values of the 9 free parameters fixing the $u$ and $d$ quark transversity distribution functions and favored and disfavored Collins fragmentation functions. The table is from ref. [152].

We also define the following variable:

$$
\left\langle P_{\perp}^{2}\right\rangle_{H_{1}^{\perp}}=\frac{\left\langle P_{\perp}^{2}\right\rangle M_{C}^{2}}{\left\langle P_{\perp}^{2}\right\rangle+M_{C}^{2}}
$$

We can rewrite the parametrizations of Collins FF as

$$
H_{1}^{\perp}\left(z, P_{\perp}^{2}\right)=H_{1}^{\perp(1)}(z) \frac{2 z^{2} m_{h}^{2}}{\pi\left\langle P_{\perp}^{2}\right\rangle_{H_{1}^{\perp}}^{2}} e^{-P_{\perp}^{2} /\left\langle P_{\perp}^{2}\right\rangle_{H_{1}^{\perp}}} .
$$

\section{A.5 Boer-Mulders function $h_{1}^{\perp q}\left(x, k_{\perp}^{2}\right)$}

The Boer-Mulders function $h_{1}^{\perp}$ [3] measures the transverse polarization asymmetry of quarks inside an unpolarized nucleon. The Boer-Mulders functions were studied phenomenologically in refs. $[157,162,163]$, we present the parameters of extractions of the Boer-Mulders function from ref. [162] in table 4.

Ref. [162] used the parametrization in which the Boer-Mulders function is proportional to the Sivers functions, such that

$$
\begin{aligned}
\left\langle k_{\perp}^{2}\right\rangle_{h_{1}^{\perp}} & =\frac{\left\langle k_{\perp}^{2}\right\rangle M_{B M}^{2}}{\left\langle k_{\perp}^{2}\right\rangle+M_{B M}^{2}}, \\
h_{1}^{\perp}\left(x, k_{\perp}^{2}\right) & =-\frac{M}{M_{B M}} \sqrt{2 e} N_{B M}^{q} N_{q}(x) f_{q / p}(x, Q) \frac{e^{-k_{\perp}^{2} /\left\langle k_{\perp}^{2}\right\rangle_{h \perp}^{\perp}}}{\pi\left\langle k_{\perp}^{2}\right\rangle},
\end{aligned}
$$

where

$$
\mathcal{N}_{q}(x)=N_{q} x^{\alpha}(1-x)^{\beta} \frac{(\alpha+\beta)^{(\alpha+\beta)}}{\alpha^{\alpha} \beta^{\beta}} .
$$

The first moment of the Boer-Mulders function is

$$
\begin{aligned}
h_{1}^{\perp(1) q}(x) & =-\frac{\sqrt{\frac{e}{2}}\left\langle k_{\perp}^{2}\right\rangle M_{B M}^{3}}{M\left(\left\langle k_{\perp}^{2}\right\rangle+M_{B M}^{2}\right)^{2}} N_{q} f_{q}(x, Q) \\
& =-\sqrt{\frac{e}{2}} \frac{1}{M M_{B M}} \frac{\left\langle k_{\perp}^{2}\right\rangle_{h_{1}^{\perp}}^{2}}{\left\langle k_{\perp}^{2}\right\rangle} N_{q} f_{q}(x, Q) .
\end{aligned}
$$

We can rewrite the parametrizations of Boer-Mulders functions as

$$
h_{1}^{\perp q}\left(x, k_{\perp}^{2}\right)=h_{1}^{\perp(1) q}(x) \frac{2 M^{2}}{\pi\left\langle k_{\perp}^{2}\right\rangle_{h_{1}^{\perp}}^{2}} e^{-k_{\perp}^{2} /\left\langle k_{\perp}^{2}\right\rangle_{h_{1}^{\perp}}} .
$$




\begin{tabular}{|crll|}
\hline$N_{B M}^{u}=2.1 \pm 0.1$ & $N_{B M}^{d}=$ & $-1.111 \pm 0.001$ & \\
$N_{u}=0.35$ & $\alpha_{u}=0.73$ & $\beta_{u}=3.46$ & \\
$N_{d}=-0.9$ & $\alpha_{d}=1.08$ & $\beta_{d}=3.46$ & $M_{B M}^{2}=0.34\left(\mathrm{GeV}^{2}\right)$ \\
\hline
\end{tabular}

Table 4. Fitted parameters (those with error bars) and fixed parameters (those without error bars) of the Boer-Mulders quark distributions from ref. [157].

$$
\begin{array}{lll}
\alpha=2.5 \pm 1.5 & \beta=2 \text { fixed } \\
N_{u}=1 \pm 1.4 & N_{d}=-1 \pm 1.3 \quad M_{T T}^{2}=0.18 \pm 0.7 \mathrm{GeV}^{2}
\end{array}
$$

Table 5. Fitted parameters of the pretzelosity quark distributions. Table from ref. [169].

\section{A.6 Pretzelosity distribution $h_{1 T}^{\perp q}\left(x, k_{\perp}^{2}\right)$}

Pretzelosity distribution function $h_{1 T}^{\perp}$ [169] describes transversely polarized quarks inside a transversely polarized nucleon. We use the following form of $h_{1 T}^{\perp a}$ [169]:

$$
h_{1 T}^{\perp a}\left(x, k_{\perp}^{2}\right)=\frac{M^{2}}{M_{T T}^{2}} e^{-k_{\perp}^{2} / M_{T T}^{2}} h_{1 T}^{\perp a}(x) \frac{e^{-k_{\perp}^{2} /\left\langle k_{\perp}^{2}\right\rangle}}{\pi\left\langle k_{\perp}^{2}\right\rangle}=\frac{M^{2}}{M_{T}^{2}} h_{1 T}^{\perp a}(x) \frac{e^{-k_{\perp}^{2} /\left\langle k_{\perp}^{2}\right\rangle_{h_{1 T}}}}{\pi\left\langle k_{\perp}^{2}\right\rangle},
$$

where

$$
\begin{aligned}
h_{1 T}^{\perp a}(x) & =e \mathcal{N}^{a}(x)\left(f_{1}^{a}(x, Q)-g_{1}^{a}(x, Q)\right), \\
\mathcal{N}^{a}(x) & =N^{a} x^{\alpha}(1-x)^{\beta} \frac{(\alpha+\beta)^{\alpha+\beta}}{\alpha^{\alpha} \beta^{\beta}}, \\
\left\langle k_{\perp}^{2}\right\rangle_{h_{1 T}^{\perp}} & =\frac{\left\langle k_{\perp}^{2}\right\rangle M_{T T}^{2}}{\left\langle k_{\perp}^{2}\right\rangle+M_{T T}^{2}},
\end{aligned}
$$

and where $N^{a}, \alpha, \beta$, and $M_{T}$ are parameters fitted to data and can be found in table 5 .

We use eq. (B.8) to obtain for the second moment of $h_{1 T}^{\perp a}\left(x, k_{\perp}^{2}\right)$ in (A.20) the result shown below, and use it to rewrite parametrization of pretzelosity functions as

$$
h_{1 T}^{\perp(2) a}(x)=\frac{h_{1 T}^{\perp a}(x)\left\langle k_{\perp}^{2}\right\rangle_{h_{1 T}^{\perp}}^{3}}{2 M^{2} M_{T T}^{2}\left\langle k_{\perp}^{2}\right\rangle}, \quad h_{1 T}^{\perp q}\left(x, k_{\perp}^{2}\right)=h_{1 T}^{\perp(2) q}(x) \frac{2 M^{4}}{\pi\left\langle k_{\perp}^{2}\right\rangle_{h_{1 T}^{\perp}}^{3}} e^{-k_{\perp}^{2} /\left\langle k_{\perp}^{2}\right\rangle_{h_{1 T}}} .
$$

\section{B Convolution integrals and expressions in Gaussian Ansatz}

In this appendix we explain the notation for convolution integrals of TMDs and FFs and give the explicit results obtained assuming the Gaussian Ansatz.

\section{B.1 Notation for convolution integrals}

Structure functions are expressed as convolutions of TMDs and FFs in the Bjorken limit at tree level. For reference we quote the convolution integrals in "Amsterdam notation" [5] $\mathcal{C}[w f D]=x \sum_{a} e_{a}^{2} \int d^{2} \boldsymbol{p}_{T} d^{2} \boldsymbol{k}_{T} \delta^{(2)}\left(\boldsymbol{p}_{T}-\boldsymbol{k}_{T}-\boldsymbol{P}_{h \perp} / z\right) w\left(\boldsymbol{p}_{T}, \boldsymbol{k}_{T}\right) f^{a}\left(x, p_{T}^{2}\right) D^{a}\left(z, z^{2} k_{T}^{2}\right)$, 
where all transverse momenta refer to the virtual photon-proton center-of-mass frame and $\hat{\boldsymbol{h}}=\boldsymbol{P}_{h \perp} / P_{h \perp}$. Hereby $\boldsymbol{p}_{T}$ is the transverse momentum of quark with respect to nucleon, $\boldsymbol{k}_{T}$ is the transverse momentum of the fragmenting quark with respect to produced hadron. The notation is not unique. The one chosen in this work, in comparison to other works, is

$$
\begin{array}{rll}
\text { transverse momentum in TMD: } & {\left[\boldsymbol{k}_{\perp}\right]_{\text {our }}=\left[\boldsymbol{k}_{\perp}\right]_{\text {ref. [219] }}=\left[\boldsymbol{p}_{T}\right]_{\text {ref. [5] }},} \\
\text { transverse momentum in FF: } & {\left[\boldsymbol{P}_{\perp}\right]_{\text {our }}=\left[\boldsymbol{p}_{\perp}\right]_{\text {ref. [219] }}=-z\left[\boldsymbol{k}_{T}\right]_{\text {ref. [5] }},} \\
\text { transverse hadron momenta: } & {\left[\boldsymbol{P}_{h T}\right]_{\text {our }}=\left[\boldsymbol{P}_{T}\right]_{\text {ref. [219] }}=\left[\boldsymbol{P}_{h \perp}\right]_{\text {ref. [5] }} .}
\end{array}
$$

Notice that $\left[\boldsymbol{P}_{\perp}\right]_{\text {our }}=-z\left[\boldsymbol{k}_{T}\right]_{\text {ref. [5] }}$ is the transverse momentum the hadron acquires in the fragmentation process. The normalization for unpolarized fragmentation functions is

$$
D_{1}^{a}(z)=\left[\int d^{2} \boldsymbol{P}_{\perp} D_{1}^{a}\left(z, P_{\perp}^{2}\right)\right]_{\mathrm{our}}=\left[z^{2} \int d^{2} \boldsymbol{k}_{T} D_{1}^{a}\left(z, z^{2} k_{T}^{2}\right)\right]_{\text {ref. [5] }} .
$$

The "Amsterdam" convolution integral (B.1) reads in our notation

$\mathcal{C}[w f D]=x \sum_{a} e_{a}^{2} \int d^{2} \boldsymbol{k}_{\perp} d^{2} \boldsymbol{P}_{\perp} \delta^{(2)}\left(z \boldsymbol{k}_{\perp}+\boldsymbol{P}_{\perp}-\boldsymbol{P}_{h T}\right) w\left(\boldsymbol{k}_{\perp},-\frac{\boldsymbol{P}_{\perp}}{z}\right) f^{a}\left(x, k_{\perp}^{2}\right) D^{a}\left(z, P_{\perp}^{2}\right)$.

\section{B.2 Gaussian Ansatz}

For a generic TMD and FF the Gaussian Ansatz is given by

$$
f^{a}\left(x, k_{\perp}^{2}\right)=f^{a}(x) \frac{\exp \left(-k_{\perp}^{2} /\left\langle k_{\perp}^{2}\right\rangle\right)}{\pi\left\langle k_{\perp}^{2}\right\rangle}, \quad D^{a}\left(z, P_{\perp}^{2}\right)=D^{a}(z) \frac{\exp \left(-P_{\perp}^{2} /\left\langle P_{\perp}^{2}\right\rangle\right)}{\pi\left\langle P_{\perp}^{2}\right\rangle}
$$

where $\left\langle k_{\perp}^{2}\right\rangle$ could be $x$-dependent, and $\left\langle P_{\perp}^{2}\right\rangle z$-dependent. Both could be flavor-dependent. The variable $P_{\perp}$ is convenient because phenomenological experience shows that $P_{\perp}$ in $D_{1}^{q / h}\left(z, P_{\perp}^{2}\right)$ exhibits a Gaussian distribution with weakly $z$-dependent Gaussian width. The distribution of transverse momenta in $\left[D^{a}\left(z, z^{2} k_{T}^{2}\right)\right]_{\text {ref. [5] }}$ would require a strongly $z$-dependent Gaussian width. It is a matter of taste which one prefers to use.

It is convenient to work with transverse moments of TMDs and FFs which are defined, and in the Gaussian model given by

$$
\begin{aligned}
f^{(n)}(x) & =\int d^{2} \boldsymbol{k}_{\perp}\left(\frac{k_{\perp}^{2}}{2 M^{2}}\right)^{n} f\left(x, k_{\perp}^{2}\right) \stackrel{\text { Gauss }}{=} \frac{n !\left\langle k_{\perp}^{2}\right\rangle^{n}}{2^{n} M_{N}^{2 n}} f(x), \\
D^{(n)}(z) & =\int d^{2} \boldsymbol{P}_{\perp}\left(\frac{P_{\perp}^{2}}{2 z^{2} m_{h}^{2}}\right)^{n} D\left(z, P_{\perp}^{2}\right) \stackrel{\text { Gauss }}{=} \frac{n !\left\langle P_{\perp}^{2}\right\rangle^{n}}{2^{n} z^{2 n} m_{h}^{2 n}} D(z) .
\end{aligned}
$$

It is important to keep in mind that these objects are well-defined in the Gaussian model. However, in QCD and even in simple models [85, 120] one faces issues with UV divergences and has to carefully define how to deal with them.

In eqs. (B.8) the Gaussian dependence is factorized from $x$ or $z$ dependence and parametrizations are made with respect to either $f(x)$ or $D(z)$. As we saw in appendix A some TMD functions are parametrized with higher moments directly as operator product expansion of TMDs may start from higher twist matrix element instead of the usual twist-2 one. In those cases equivalent formulas to eqs. (B.8) can be easily derived. 


\section{B.3 Gaussian Ansatz for the derived TMDs used in this work}

Having discussed the Gaussian Ansatz for the 8 basis functions in eqs. (4.5a)-(4.5h) of section 4.4 and in appendix A, we list here the Ansätze for the following derived TMDs:

$$
\begin{aligned}
g_{1 T}^{\perp q}\left(x, k_{\perp}^{2}\right) & =g_{1 T}^{\perp(1) q}(x) \frac{2 M_{N}^{2}}{\pi\left\langle k_{\perp}^{2}\right\rangle_{g_{1 T}^{\perp}}^{2}} e^{-k_{\perp}^{2} /\left\langle k_{\perp}^{2}\right\rangle_{g_{1 T}^{\perp}}}, & & \text { cf. section } 6.1, \\
h_{1 L}^{\perp a}\left(x, k_{\perp}^{2}\right) & =h_{1 L}^{\perp(1) a}(x) \frac{2 M_{N}^{2}}{\pi\left\langle k_{\perp}^{2}\right\rangle_{h_{1 L}^{\perp}}^{2}} e^{-k_{\perp}^{2} /\left\langle k_{\perp}^{2}\right\rangle_{h_{1 L}^{\perp}}} & & \text { cf. section } 6.2, \\
g_{T}^{q}\left(x, k_{\perp}^{2}\right) & =g_{T}^{q}(x) \frac{1}{\pi\left\langle k_{\perp}^{2}\right\rangle_{T}} e^{-k_{\perp}^{2} /\left\langle k_{\perp}^{2}\right\rangle_{g_{T}}}, & & \text { cf. section } 7.2, \\
g_{T}^{\perp q}\left(x, k_{\perp}^{2}\right) & =g_{T}^{\perp(2) q}(x) \frac{2 M^{4}}{\pi\left\langle k_{\perp}^{2}\right\rangle_{g_{T}^{\perp}}^{3}} e^{-k_{\perp}^{2} /\left\langle k_{\perp}^{2}\right\rangle_{g_{T}^{\perp}}}, & & \text { cf. section } 7.3, \\
g_{L}^{\perp q}\left(x, k_{\perp}^{2}\right) & =g_{L}^{\perp(1) q}(x) \frac{2 M_{N}^{2}}{\pi\left\langle k_{\perp}^{2}\right\rangle_{g_{L}^{\perp}}^{2}} e^{-k_{\perp}^{2} /\left\langle k_{\perp}^{2}\right\rangle_{g_{L}}}, & & \text { cf. section } 7.4, \\
h_{L}^{q}\left(x, k_{\perp}^{2}\right) & =h_{L}^{q}(x) \frac{1}{\pi\left\langle k_{\perp}^{2}\right\rangle_{h_{L}}} e^{-k_{\perp}^{2} /\left\langle k_{\perp}^{2}\right\rangle_{L}}, & & \text { cf. section } 7.5, \\
h_{T}^{\perp q}\left(x, k_{\perp}^{2}\right) & =h_{T}^{\perp(1) q}(x) \frac{2 M^{2}}{\pi\left\langle k_{\perp}^{2}\right\rangle_{h_{T}^{\perp}}^{2}} e^{-k_{\perp}^{2} /\left\langle k_{\perp}^{2}\right\rangle_{h_{T}}}, & & \text { cf. section } 7.6, \\
h_{T}^{q}\left(x, k_{\perp}^{2}\right) & =h_{T}^{(1) q}(x) \frac{2 M^{2}}{\pi\left\langle k_{\perp}^{2}\right\rangle_{h_{T}}^{2}} e^{-k_{\perp}^{2} /\left\langle k_{\perp}^{2}\right\rangle_{h_{T}}}, & & \text { cf. section } 7.6, \\
f_{T}^{\perp q}\left(x, k_{\perp}^{2}\right) & =f_{T}^{\perp(2) q}(x) \frac{2 M^{4}}{\pi\left\langle k_{\perp}^{2}\right\rangle_{f_{T}^{\perp}}^{3}} e^{-k_{\perp}^{2} /\left\langle k_{\perp}^{2}\right\rangle_{f_{T}}}, & & \text { cf. section } 7.7, \\
f^{\perp q}\left(x, k_{\perp}^{2}\right) & =f^{\perp(1) q}(x) \frac{2 M^{2}}{\pi\left\langle k_{\perp}^{2}\right\rangle_{f}^{2}} e^{-k_{\perp}^{2} /\left\langle k_{\perp}^{2}\right\rangle_{f}}, & & \text { cf. section } 7.8,
\end{aligned}
$$

\section{B.4 Comment on TMDs subject to the sum rules (2.13)}

In this section we comment on the twist-3 TMDs $f_{T}^{q}\left(x, k_{\perp}^{2}\right), h^{q}\left(x, k_{\perp}^{2}\right), e_{L}^{q}\left(x, k_{\perp}^{2}\right)$, which are T-odd, appear in the decompositions of the correlator with no explicit $k_{\perp}^{j}$-prefactors, and would have collinear PDF counterparts. But T-odd PDFs are forbidden by timereversal and parity invariance of strong interactions, which dictate the sum rules (2.13), see section 3.8. Such TMDs could be described by functions with a node in $k_{\perp},{ }^{11}$ such that they can integrate to zero in eq. (2.13). A single Gaussian has no node and is not adequate

\footnotetext{
${ }^{11}$ The possibility of TMDs with nodes is not unrealistic. For instance in the covariant parton model the helicity TMDs exhibit nodes for the $u$ - and $d$-flavor [96]. We will have to revise our description of $g_{1}^{q}\left(x, k_{\perp}^{2}\right)$ in eq. (4.5c) and appendix A.2 to something of the type (B.10), if that prediction is confirmed experimentally.
} 
for that. However, one could work with a superposition of Gaussians with different widths,

$$
\begin{aligned}
x f_{T}^{q}\left(x, k_{\perp}^{2}\right)=-f_{1 T}^{\perp(1) q}(x) & \sum_{i=1}^{n} a_{i} \frac{\exp \left(-k_{\perp}^{2} /\left\langle k_{\perp}^{2}\right\rangle_{i}\right)}{\pi\left\langle k_{\perp}^{2}\right\rangle_{i}}, \\
& \sum_{i=1}^{n} a_{i}=0,\left\langle k_{\perp}^{2}\right\rangle_{i} \neq\left\langle k_{\perp}^{2}\right\rangle_{j} \forall i \neq j, 1 \leq i, j \leq n, n \geq 2 .
\end{aligned}
$$

Notice that in (B.10) we cannot write " $f_{T}^{q}(x)$ ", which would be zero according to (2.13), and we explore here the WW-type approximation $(3.4 \mathrm{~g})$. The minimal choice would be $n=2$ with $a_{1}=-a_{2}=1$ and $\left\langle k_{\perp}^{2}\right\rangle_{1}=\left\langle k_{\perp}^{2}\right\rangle_{f_{1 T}^{\perp}}$ to make use of the theoretical guidance provided by the WW-type approximation $(3.4 \mathrm{~g})$. The second Gaussian width $\left\langle k_{\perp}^{2}\right\rangle_{2}$ could be chosen very large $\left\langle k_{\perp}^{2}\right\rangle_{2} \gg\left\langle k_{\perp}^{2}\right\rangle_{f_{1}^{\perp}}$ to model the Gaussian description of $f_{T}^{q}\left(x, k_{\perp}^{2}\right)$ similar

to that of $f_{1 T}^{\perp(1) q}\left(x, k_{\perp}^{2}\right)$ at intermediate $k_{\perp}$. A very large parameter $\left\langle k_{\perp}^{2}\right\rangle_{2}$ could be thought of as a relict which enters in the sum rule (2.13) where the $k_{\perp}$-integration formally extends up to infinity where the TMD description does not apply. The theoretical understanding of higher-twist TMDs is too limited at the present stage, but in principle this could be a pragmatic way of modeling the TMD $f_{T}^{q}\left(x, k_{\perp}^{2}\right)$ and analogously $h^{q}\left(x, k_{\perp}^{2}\right), e_{L}^{q}\left(x, k_{\perp}^{2}\right)$.

\section{B.5 Convolution integrals in Gaussian Ansatz}

Solving the convolution integrals relevant for SIDIS in the Gaussian Ansatz yields

$$
\begin{aligned}
\mathcal{C}\left[\omega^{\{0\}} f D\right] & =u \mathcal{G}\left(P_{h T}\right) \\
\mathcal{C}\left[\omega_{\mathrm{A}}^{\{1\}} f D\right] & =u \mathcal{G}\left(P_{h T}\right)\left(\frac{z P_{h T}}{m_{h}}\right) \frac{\left\langle P_{\perp}^{2}\right\rangle}{z^{2} \lambda} \\
\mathcal{C}\left[\omega_{\mathrm{B}}^{\{1\}} f D\right] & =-u \mathcal{G}\left(P_{h T}\right)\left(\frac{z P_{h T}}{M_{N}}\right) \frac{\left\langle k_{\perp}^{2}\right\rangle}{\lambda} \\
\mathcal{C}\left[\omega_{\mathrm{A}}^{\{2\}} f D\right] & =u \mathcal{G}\left(P_{h T}\right) \frac{\left\langle k_{\perp}^{2}\right\rangle\left\langle P_{\perp}^{2}\right\rangle}{\lambda M_{N} m_{h}}\left(-1+\frac{2 P_{h T}^{2}}{\lambda}\right) \\
\mathcal{C}\left[\omega_{\mathrm{B}}^{\{2\}} f D\right] & =u \mathcal{G}\left(P_{h T}\right) \frac{\left\langle k_{\perp}^{2}\right\rangle\left\langle P_{\perp}^{2}\right\rangle}{\lambda M_{N} m_{h}}\left(1-\frac{P_{h T}^{2}}{\lambda}\right) \\
\mathcal{C}\left[\omega_{\mathrm{AB}}^{\{2\}} f D\right] & =u \mathcal{G}\left(P_{h T}\right)\left(\frac{z P_{h T}}{M_{N}}\right)\left(\frac{z P_{h T}}{m_{h}}\right) \frac{\left\langle k_{\perp}^{2}\right\rangle}{\lambda} \frac{\left\langle P_{\perp}^{2}\right\rangle}{z^{2} \lambda} \\
\mathcal{C}\left[\omega_{\mathrm{C}}^{\{2\}} f D\right] & =\frac{u}{2} \mathcal{G}\left(P_{h T}\right)\left(\frac{z P_{h T}}{M_{N}}\right)\left(\frac{z P_{h T}}{M_{N}}\right) \frac{\left\langle k_{\perp}^{2}\right\rangle}{\lambda} \frac{\left\langle k_{\perp}^{2}\right\rangle}{\lambda} \\
\mathcal{C}\left[\omega^{\{3\}} f D\right] & =\frac{u}{2} \mathcal{G}\left(P_{h T}\right)\left(\frac{z P_{h T}}{M_{N}}\right)\left(\frac{z P_{h T}}{M_{N}}\right)\left(\frac{z P_{h T}}{m_{h}}\right) \frac{\left\langle k_{\perp}^{2}\right\rangle}{\lambda} \frac{\left\langle k_{\perp}^{2}\right\rangle}{\lambda} \frac{\left\langle P_{\perp}^{2}\right\rangle}{z^{2} \lambda}
\end{aligned}
$$

with the $\omega_{i}^{\{n\}}$ as defined in eq. (2.19), and we introduced the abbreviations

$$
u=x \sum_{a} e_{a}^{2} f^{a}(x) D^{a}(z), \quad \mathcal{G}\left(P_{h T}\right)=\frac{\exp \left(-P_{h T}^{2} / \lambda\right)}{\pi \lambda}, \quad \lambda=z^{2}\left\langle k_{\perp}^{2}\right\rangle+\left\langle P_{\perp}^{2}\right\rangle,
$$

with the normalization $\int d^{2} P_{h T} \mathcal{G}\left(P_{h T}\right)=1$. It is important to keep in mind that strictly speaking $\mathcal{G}\left(P_{h T}\right)=\mathcal{G}\left(P_{h T}, x, z\right)$ also depends on $x$ and $z$. The "non-compact" notation in 
eqs. (B.11) was chosen to display the pattern. The masses $M_{N}$ or $m_{h}$ in the denominators of the $P_{h T}$ indicate the "origins" of the contributions: due to intrinsic $k_{\perp}$ from target, due to transverse momenta $P_{\perp}$ acquired during fragmentation, or both. The weight $\omega_{\mathrm{B}}^{\{2\}}$ is the only which enters cross sections and does not have a homogeneous scaling in $P_{h T}$.

For practical application it is convenient to absorb as many (Gaussian model) parameters as possible into expressions that can be more easily fitted to data. One way to achieve this is to make use of the transverse moments (B.8). We introduce the following abbreviations

$$
\begin{array}{rlrl}
u_{\mathrm{A}}^{\{1\}} & =x \sum_{a} e_{a}^{2} f^{a}(x) D^{(1) a}(z), & & u_{\mathrm{B}}^{\{1\}}=x \sum_{a} e_{a}^{2} f^{(1) a}(x) D^{a}(z), \\
u_{\mathrm{AB}}^{\{2\}}=x \sum_{a} e_{a}^{2} f^{(1) a}(x) D^{(1) a}(z), & & u_{\mathrm{C}}^{\{2\}}=x \sum_{a} e_{a}^{2} f^{(2) a}(x) D^{a}(z), \\
u_{\mathrm{C}}^{\{3\}} & =x \sum_{a} e_{a}^{2} f^{(2) a}(x) D^{(1) a}(z) . & &
\end{array}
$$

In this notation the results in eqs. (B.11) read

$$
\begin{aligned}
\mathcal{C}\left[\omega_{\mathrm{A}}^{\{1\}} f D\right] & =u_{\mathrm{A}}^{(1)} \mathcal{G}\left(P_{h T}\right)\left(\frac{z P_{h T}}{m_{h}}\right) \frac{2 m_{h}^{2}}{\lambda} \\
\mathcal{C}\left[\omega_{\mathrm{B}}^{\{1\}} f D\right] & =-u_{\mathrm{B}}^{(1)} \mathcal{G}\left(P_{h T}\right)\left(\frac{z P_{h T}}{M_{N}}\right) \frac{2 M_{N}^{2}}{\lambda} \\
\mathcal{C}\left[\omega_{\mathrm{B}}^{\{2\}} f D\right] & =u_{\mathrm{B}}^{(2)} \mathcal{G}\left(P_{h T}\right) \frac{4 z^{2} m_{h} M_{N}}{\lambda}\left(1-\frac{P_{h T}^{2}}{\lambda}\right) \\
\mathcal{C}\left[\omega_{\mathrm{AB}}^{\{2\}} f D\right] & =u_{\mathrm{AB}}^{(2)} \mathcal{G}\left(P_{h T}\right)\left(\frac{z P_{h T}}{M_{N}}\right)\left(\frac{z P_{h T}}{m_{h}}\right) \frac{2 M_{N}^{2}}{\lambda} \frac{2 m_{h}^{2}}{\lambda} \\
\mathcal{C}\left[\omega_{\mathrm{C}}^{\{2\}} f D\right] & =\frac{u_{\mathrm{C}}^{(2)}}{2} \mathcal{G}\left(P_{h T}\right)\left(\frac{z P_{h T}}{M_{N}}\right)\left(\frac{z P_{h T}}{M_{N}}\right) \frac{2 M_{N}^{2}}{\lambda} \frac{2 M_{N}^{2}}{\lambda} \\
\mathcal{C}\left[\omega^{\{3\}} f D\right] & =\frac{u^{(3)}}{2} \mathcal{G}\left(P_{h T}\right)\left(\frac{z P_{h T}}{M_{N}}\right)\left(\frac{z P_{h T}}{M_{N}}\right)\left(\frac{z P_{h T}}{m_{h}}\right) \frac{2 M_{N}^{2}}{\lambda} \frac{2 M_{N}^{2}}{\lambda} \frac{2 m_{h}^{2}}{\lambda}
\end{aligned}
$$

In this notation the results in eqs. (B.11) read

$$
\mathcal{C}\left[\omega_{i}^{\{n\}} f D\right]=u_{i}^{(n)} \mathcal{G}\left(P_{h T}\right) \times\left[\delta_{n 2} \delta_{i \mathrm{~B}} a_{B}^{(2)}+b_{i}^{(n)}\left(\frac{z P_{h T}}{\lambda}\right)^{n}\right]
$$

with

$$
\begin{aligned}
& b^{(0)}=1 \text {, } \\
& b_{\mathrm{A}}^{(1)}=2 m_{h}, \quad b_{\mathrm{B}}^{(1)}=2 M_{N}, \\
& a_{\mathrm{B}}^{(2)}=4 M_{N} m_{h} \lambda^{-1} z^{2}, \quad b_{\mathrm{AB}}^{(2)}=-b_{\mathrm{B}}^{(2)}=4 M_{N} m_{h}, \quad b_{\mathrm{C}}^{(2)}=M_{N}^{2} \text {, } \\
& b^{(3)}=2 M_{N}^{2} m_{h} \text {. }
\end{aligned}
$$

Finally, integrating out transverse hadron momenta yields

$$
\int d^{2} P_{h T} \mathcal{C}\left[\omega_{i}^{\{n\}} f D\right]=u_{i}^{(n)} c_{i}^{(n)}\left(\frac{z}{\lambda^{1 / 2}}\right)^{n}
$$


with

$$
\begin{aligned}
c^{(0)} & =1, & & \\
c_{\mathrm{A}}^{(1)} & =\sqrt{\pi} m_{h}, & c_{\mathrm{B}}^{(1)}=\sqrt{\pi} M_{N}, & \\
c_{\mathrm{AB}}^{(2)} & =4 M_{N} m_{h}, & c_{\mathrm{C}}^{(2)}=M_{N}^{2}, & c_{\mathrm{B}}^{(2)}=0, \\
c^{(3)} & =\frac{3}{2} \sqrt{\pi} M_{N}^{2} m_{h} . & &
\end{aligned}
$$

Open Access. This article is distributed under the terms of the Creative Commons Attribution License (CC-BY 4.0), which permits any use, distribution and reproduction in any medium, provided the original author(s) and source are credited.

\section{References}

[1] A. Kotzinian, New quark distributions and semiinclusive electroproduction on the polarized nucleons, Nucl. Phys. B 441 (1995) 234 [hep-ph/9412283] [inSPIRE].

[2] P.J. Mulders and R.D. Tangerman, The complete tree-level result up to order $1 / Q$ for polarized deep-inelastic leptoproduction, Nucl. Phys. B 461 (1996) 197 [Erratum ibid. B 484 (1997) 538] [hep-ph/9510301] [INSPIRE].

[3] D. Boer and P.J. Mulders, Time-reversal odd distribution functions in leptoproduction, Phys. Rev. D 57 (1998) 5780 [hep-ph/9711485] [INSPIRE].

[4] K. Goeke, A. Metz and M. Schlegel, Parameterization of the quark-quark correlator of a spin-1/2 hadron, Phys. Lett. B 618 (2005) 90 [hep-ph/0504130] [INSPIRE].

[5] A. Bacchetta, M. Diehl, K. Goeke, A. Metz, P.J. Mulders and M. Schlegel, Semi-inclusive deep inelastic scattering at small transverse momentum, JHEP 02 (2007) 093 [hep-ph/0611265] [INSPIRE].

[6] F. Stock et al., The FILTEX/HERMES polarized hydrogen atomic beam source, Nucl. Instrum. Meth. A 343 (1994) 334 [INSPIRE].

[7] HERMES collaboration, The HERMES polarized hydrogen and deuterium gas target in the HERA electron storage ring, Nucl. Instrum. Meth. A 540 (2005) 68 [physics/0408137] [INSPIRE].

[8] D.G. Crabb and W. Meyer, Solid polarized targets for nuclear and particle physics experiments, Ann. Rev. Nucl. Part. Sci. 47 (1997) 67 [InSPIRE].

[9] S. Goertz, W. Meyer and G. Reicherz, Polarized H, D and ${ }^{3}$ He targets for particle physics experiments, Prog. Part. Nucl. Phys. 49 (2002) 403 [Erratum ibid. 51 (2003) 309] [InSPIRE].

[10] S. Arnold, A. Metz and M. Schlegel, Dilepton production from polarized hadron hadron collisions, Phys. Rev. D 79 (2009) 034005 [arXiv:0809.2262] [INSPIRE].

[11] A. Metz and A. Vossen, Parton fragmentation functions, Prog. Part. Nucl. Phys. 91 (2016) 136 [arXiv: 1607.02521] [INSPIRE].

[12] G.A. Miller, Densities, parton distributions, and measuring the nonspherical shape of the nucleon, Phys. Rev. C 76 (2007) 065209 [arXiv:0708.2297] [INSPIRE].

[13] M. Burkardt, Spin-orbit correlations and single-spin asymmetries, in Workshop on Exclusive Reactions at High Momentum Transfer, Newport News, Virginia, May 21-24, 2007 (2007) [DOI:10.1142/9789812796950_0006] [arXiv:0709.2966] [INSPIRE]. 
[14] M. Burkardt, The ge Structure Function, AIP Conf. Proc. 1155 (2009) 26 [arXiv:0905.4079] [INSPIRE].

[15] A. Bacchetta, M. Boglione, A. Henneman and P.J. Mulders, Bounds on transverse momentum dependent distribution and fragmentation functions, Phys. Rev. Lett. 85 (2000) 712 [hep-ph/9912490] [INSPIRE].

[16] S. Wandzura and F. Wilczek, Sum rules for spin-dependent electroproduction: Test of relativistic constituent quarks, Phys. Lett. 72B (1977) 195 [INSPIRE].

[17] R.L. Jaffe and X.-D. Ji, Chiral-odd parton distributions and Drell-Yan processes, Nucl. Phys. B 375 (1992) 527 [INSPIRE].

[18] R.L. Jaffe, $g_{2}$-The Nucleon's Other Spin-Dependent Structure Function, Comments Nucl. Part. Phys. 19 (1990) 239 [inSPIRE].

[19] J. Balla, M.V. Polyakov and C. Weiss, Nucleon matrix elements of higher-twist operators from the instanton vacuum, Nucl. Phys. B 510 (1998) 327 [hep-ph/9707515] [InSPIRE].

[20] B. Dressler and M.V. Polyakov, Twist-three contribution to $h_{L}$ in the instanton vacuum, Phys. Rev. D 61 (2000) 097501 [hep-ph/9912376] [INSPIRE].

[21] M. Göckeler et al., Lattice calculation of the nucleon's spin-dependent structure function $g_{2}$ reexamined, Phys. Rev. D 63 (2001) 074506 [hep-lat/0011091] [INSPIRE].

[22] M. Göckeler et al., Investigation of the second moment of the nucleon's $g_{1}$ and $g_{2}$ structure functions in two-flavor lattice QCD, Phys. Rev. D 72 (2005) 054507 [hep-lat/0506017] [INSPIRE].

[23] E143 collaboration, Measurements of the proton and deuteron spin structure functions $g_{1}$ and $g_{2}$, Phys. Rev. D 58 (1998) 112003 [hep-ph/9802357] [INSPIRE].

[24] E155 collaboration, Precision measurement of the proton and deuteron spin structure functions $g_{2}$ and asymmetries $A_{2}$, Phys. Lett. B 553 (2003) 18 [hep-ex/0204028] [INSPIRE].

[25] HERMES collaboration, Measurement of the virtual-photon asymmetry $A_{2}$ and the spin-structure function $g_{2}$ of the proton, Eur. Phys. J. C 72 (2012) 1921 [arXiv: 1112.5584] [INSPIRE].

[26] A.M. Kotzinian and P.J. Mulders, Longitudinal quark polarization in transversely polarized nucleons, Phys. Rev. D 54 (1996) 1229 [hep-ph/9511420] [INSPIRE].

[27] A.M. Kotzinian and P.J. Mulders, Probing transverse quark polarization via azimuthal asymmetries in leptoproduction, Phys. Lett. B 406 (1997) 373 [hep-ph/9701330] [INSPIRE].

[28] A. Kotzinian, B. Parsamyan and A. Prokudin, Predictions for double spin asymmetry $A_{L T}$ in semiinclusive DIS, Phys. Rev. D 73 (2006) 114017 [hep-ph/0603194] [INSPIRE].

[29] H. Avakian, A.V. Efremov, K. Goeke, A. Metz, P. Schweitzer and T. Teckentrup, Are there approximate relations among transverse momentum dependent distribution functions?, Phys. Rev. D 77 (2008) 014023 [arXiv:0709.3253] [INSPIRE].

[30] A. Metz, P. Schweitzer and T. Teckentrup, Lorentz invariance relations between parton distributions and the Wandzura-Wilczek approximation, Phys. Lett. B 680 (2009) 141 [arXiv: 0810.5212] [INSPIRE].

[31] T. Teckentrup, A. Metz and P. Schweitzer, Lorentz invariance relations and Wandzura-Wilczek approximation, Mod. Phys. Lett. A 24 (2009) 2950 [arXiv:0910.2567] [INSPIRE].

[32] R.D. Tangerman and P.J. Mulders, Polarized twist-three distributions $g_{T}$ and $h_{L}$ and the role of intrinsic transverse momentum, hep-ph/9408305 [INSPIRE]. 
[33] H. Avakian, H. Matevosyan, B. Pasquini and P. Schweitzer, Studying the information content of TMDs using Monte Carlo generators, J. Phys. G 42 (2015) 034015 [INSPIRE].

[34] A. Metz and M. Schlegel, Twist-3 single-spin asymmetries in semi-inclusive deep-inelastic scattering, Eur. Phys. J. A 22 (2004) 489 [hep-ph/0403182] [INSPIRE].

[35] L.P. Gamberg, D.S. Hwang, A. Metz and M. Schlegel, Light-cone divergence in twist-3 correlation functions, Phys. Lett. B 639 (2006) 508 [hep-ph/0604022] [INSPIRE].

[36] J.C. Collins, Leading-twist single-transverse-spin asymmetries: Drell-Yan and deep-inelastic scattering, Phys. Lett. B 536 (2002) 43 [hep-ph/0204004] [INSPIRE].

[37] J.P. Ralston and D.E. Soper, Production of Dimuons from High-Energy Polarized Proton Proton Collisions, Nucl. Phys. B 152 (1979) 109 [INSPIRE].

[38] J.C. Collins and D.E. Soper, Back-To-Back Jets in QCD, Nucl. Phys. B 193 (1981) 381 [Erratum ibid. B 213 (1983) 545] [INSPIRE].

[39] X.-d. Ji, J.-p. Ma and F. Yuan, QCD factorization for semi-inclusive deep-inelastic scattering at low transverse momentum, Phys. Rev. D 71 (2005) 034005 [hep-ph/0404183] [INSPIRE].

[40] X.-d. Ji, J.-P. Ma and F. Yuan, QCD factorization for spin-dependent cross sections in DIS and Drell-Yan processes at low transverse momentum, Phys. Lett. B 597 (2004) 299 [hep-ph/0405085] [INSPIRE].

[41] J. Collins, Foundations of perturbative QCD, Camb. Monogr. Part. Phys. Nucl. Phys. Cosmol. 32 (2011) 1 [INSPIRE].

[42] M.G. Echevarría, A. Idilbi and I. Scimemi, Soft and collinear factorization and transverse momentum dependent parton distribution functions, Phys. Lett. B 726 (2013) 795 [arXiv:1211.1947] [INSPIRE].

[43] S.M. Aybat and T.C. Rogers, TMD Parton Distribution and Fragmentation Functions with QCD Evolution, Phys. Rev. D 83 (2011) 114042 [arXiv:1101.5057] [INSPIRE].

[44] S.M. Aybat, J.C. Collins, J.-W. Qiu and T.C. Rogers, The QCD Evolution of the Sivers Function, Phys. Rev. D 85 (2012) 034043 [arXiv:1110.6428] [inSPIRE].

[45] M.G. Echevarria, A. Idilbi, Z.-B. Kang and I. Vitev, QCD Evolution of the Sivers Asymmetry, Phys. Rev. D 89 (2014) 074013 [arXiv:1401.5078] [InSPIRE].

[46] J. Collins and T. Rogers, Understanding the large-distance behavior of transverse-momentum-dependent parton densities and the Collins-Soper evolution kernel, Phys. Rev. D 91 (2015) 074020 [arXiv:1412.3820] [INSPIRE].

[47] J.P. Ma and G.P. Zhang, QCD corrections of all structure functions in transverse momentum dependent factorization for Drell-Yan processes, JHEP 02 (2014) 100 [arXiv: 1308.2044] [INSPIRE].

[48] S.M. Aybat, A. Prokudin and T.C. Rogers, Calculation of TMD Evolution for Transverse Single Spin Asymmetry Measurements, Phys. Rev. Lett. 108 (2012) 242003 [arXiv:1112.4423] [INSPIRE].

[49] Z.-B. Kang, A. Prokudin, P. Sun and F. Yuan, Extraction of quark transversity distribution and Collins fragmentation functions with QCD evolution, Phys. Rev. D 93 (2016) 014009 [arXiv: 1505.05589] [INSPIRE].

[50] D. Gutiérrez-Reyes, I. Scimemi and A.A. Vladimirov, Twist-2 matching of transverse momentum dependent distributions, Phys. Lett. B 769 (2017) 84 [arXiv:1702.06558] [INSPIRE]. 
[51] D. Gutierrez-Reyes, I. Scimemi, W.J. Waalewijn and L. Zoppi, Transverse-Momentum-Dependent Distributions with Jets, Phys. Rev. Lett. 121 (2018) 162001 [arXiv: 1807.07573] [INSPIRE].

[52] I. Scimemi and A. Vladimirov, Matching of transverse momentum dependent distributions at twist-3, Eur. Phys. J. C 78 (2018) 802 [arXiv:1804.08148] [INSPIRE].

[53] A.P. Chen and J.P. Ma, Light-cone singularities and transverse-momentum-dependent factorization at twist-3, Phys. Lett. B 768 (2017) 380 [arXiv:1610.08634] [INSPIRE].

[54] A.V. Efremov and P. Schweitzer, The chirally-odd twist-3 distribution $e^{a}(x)$, JHEP 08 (2003) 006 [hep-ph/0212044] [INSPIRE].

[55] R.L. Jaffe, Spin, twist and hadron structure in deep inelastic processes, in The spin structure of the nucleon. Proceedings, International School of Nucleon Structure, 1st Course, Erice, Italy, August 3-10, 1995, pp. 42-129 (1996) [hep-ph/9602236] [INSPIRE].

[56] K. Kanazawa, Y. Koike, A. Metz, D. Pitonyak and M. Schlegel, Operator constraints for twist-3 functions and Lorentz invariance properties of twist-3 observables, Phys. Rev. D 93 (2016) 054024 [arXiv: 1512.07233] [InSPIRE].

[57] E.V. Shuryak, The role of instantons in quantum chromodynamics: (I). Physical vacuum, Nucl. Phys. B 203 (1982) 93 [inSPIRE].

[58] D. Diakonov and V.Y. Petrov, Instanton-based vacuum from Feynman variational principle, Nucl. Phys. B 245 (1984) 259 [inSPIRE].

[59] D. Diakonov, M.V. Polyakov and C. Weiss, Hadronic matrix elements of gluon operators in the instanton vacuum, Nucl. Phys. B 461 (1996) 539 [hep-ph/9510232] [INSPIRE].

[60] Spin Muon (SMC) collaboration, Spin asymmetry in muon-proton deep inelastic scattering on a transversely polarized target, Phys. Lett. B 336 (1994) 125 [hep-ex/9408001] [inSPIRE].

[61] A. Accardi, A. Bacchetta, W. Melnitchouk and M. Schlegel, What can break the Wandzura-Wilczek relation?, JHEP 11 (2009) 093 [arXiv:0907.2942] [INSPIRE].

[62] M. Glück, E. Reya and A. Vogt, Dynamical parton distributions revisited, Eur. Phys. J. C 5 (1998) 461 [hep-ph/9806404] [INSPIRE].

[63] A.V. Efremov, K. Goeke and P. Schweitzer, Collins effect in semiinclusive deeply inelastic scattering and in electron-positron-annihilation, Phys. Rev. D 73 (2006) 094025 [hep-ph/0603054] [INSPIRE].

[64] M. Anselmino et al., Transversity and Collins functions from SIDIS and $e^{+} e^{-}$data, Phys. Rev. D 75 (2007) 054032 [hep-ph/0701006] [INSPIRE].

[65] M. Anselmino et al., Update on transversity and Collins functions from SIDIS and $e^{+} e^{-}$ data, Nucl. Phys. Proc. Suppl. 191 (2009) 98 [arXiv:0812.4366] [InSPIRE].

[66] Y. Koike, K. Tanaka and S. Yoshida, Drell-Yan double-spin asymmetry $A_{L T}$ in polarized p $\bar{p}$ collisions: Wandzura-Wilczek contribution, Phys. Lett. B 668 (2008) 286 [arXiv:0805.2289] [INSPIRE].

[67] P. Hägler, B.U. Musch, J.W. Negele and A. Schafer, Intrinsic quark transverse momentum in the nucleon from lattice QCD, EPL 88 (2009) 61001 [arXiv:0908.1283] [INSPIRE].

[68] B.U. Musch, P. Hägler, J.W. Negele and A. Schäfer, Exploring quark transverse momentum distributions with lattice QCD, Phys. Rev. D 83 (2011) 094507 [arXiv:1011.1213] [INSPIRE]. 
[69] C. Alexandrou et al., Computation of parton distributions from the quasi-PDF approach at the physical point, EPJ Web Conf. 175 (2018) 14008 [arXiv:1710.06408] [INSPIRE].

[70] T. Ishikawa, Y.-Q. Ma, J.-W. Qiu and S. Yoshida, Renormalizability of quasiparton distribution functions, Phys. Rev. D 96 (2017) 094019 [arXiv:1707.03107] [InSPIRE].

[71] M. Engelhardt, P. Hägler, B. Musch, J. Negele and A. Schäfer, Lattice QCD study of the Boer-Mulders effect in a pion, Phys. Rev. D 93 (2016) 054501 [arXiv:1506.07826] [INSPIRE].

[72] X. Ji, P. Sun, X. Xiong and F. Yuan, Soft factor subtraction and transverse momentum dependent parton distributions on the lattice, Phys. Rev. D 91 (2015) 074009 [arXiv: 1405.7640] [INSPIRE].

[73] B.U. Musch, P. Hägler, M. Engelhardt, J.W. Negele and A. Schafer, Sivers and Boer-Mulders observables from lattice QCD, Phys. Rev. D 85 (2012) 094510 [arXiv:1111.4249] [INSPIRE].

[74] X. Ji, J.-H. Zhang and Y. Zhao, Renormalization in Large Momentum Effective Theory of Parton Physics, Phys. Rev. Lett. 120 (2018) 112001 [arXiv:1706.08962] [INSPIRE].

[75] B. Yoon et al., Nucleon transverse-momentum-dependent parton distributions in lattice QCD: Renormalization patterns and discretization effects, Phys. Rev. D 96 (2017) 094508 [arXiv: 1706. 03406] [INSPIRE].

[76] J. Green, K. Jansen and F. Steffens, Nonperturbative Renormalization of Nonlocal Quark Bilinears for Parton Quasidistribution Functions on the Lattice Using an Auxiliary Field, Phys. Rev. Lett. 121 (2018) 022004 [arXiv: 1707.07152] [INSPIRE].

[77] J.-H. Zhang, J.-W. Chen, X. Ji, L. Jin and H.-W. Lin, Pion distribution amplitude from lattice QCD, Phys. Rev. D 95 (2017) 094514 [arXiv:1702.00008] [INSPIRE].

[78] LHPC and SESAM collaborations, Transverse structure of nucleon parton distributions from lattice QCD, Phys. Rev. Lett. 93 (2004) 112001 [hep-lat/0312014] [INSPIRE].

[79] LHPC collaboration, Nucleon generalized parton distributions from full lattice QCD, Phys. Rev. D 77 (2008) 094502 [arXiv: 0705.4295] [INSPIRE].

[80] QCDSF and UKQCD collaborations, Quark helicity flip generalized parton distributions from two-flavor lattice QCD, Phys. Lett. B 627 (2005) 113 [hep-lat/0507001] [INSPIRE].

[81] M. Burkardt and Y. Koike, Violation of sum rules for twist-3 parton distributions in QCD, Nucl. Phys. B 632 (2002) 311 [hep-ph/0111343] [INSPIRE].

[82] S.D. Bass, Fixed poles, polarized glue and nucleon spin structure, Acta Phys. Polon. B 34 (2003) 5893 [hep-ph/0311174] [INSPIRE].

[83] M. Stratmann, Bag model predictions for polarized structure functions and their $Q^{2}$-evolutions, Z. Phys. C 60 (1993) 763 [InSPIRE].

[84] A.I. Signal, Calculations of higher twist distribution functions in the MIT bag model, Nucl. Phys. B 497 (1997) 415 [hep-ph/9610480] [INSPIRE].

[85] H. Avakian, A.V. Efremov, P. Schweitzer and F. Yuan, The transverse momentum dependent distribution functions in the bag model, Phys. Rev. D 81 (2010) 074035 [arXiv: 1001.5467] [INSPIRE].

[86] R. Jakob, P.J. Mulders and J. Rodrigues, Modeling quark distribution and fragmentation functions, Nucl. Phys. A 626 (1997) 937 [hep-ph/9704335] [INSPIRE].

[87] M. Wakamatsu, Polarized structure functions $g_{2}(x)$ in the chiral quark soliton model, Phys. Lett. B 487 (2000) 118 [hep-ph/0006212] [INSPIRE]. 
[88] B. Pasquini, S. Cazzaniga and S. Boffi, Transverse momentum dependent parton distributions in a light-cone quark model, Phys. Rev. D 78 (2008) 034025 [arXiv:0806.2298] [INSPIRE].

[89] C. Lorce, B. Pasquini and M. Vanderhaeghen, Unified framework for generalized and transverse-momentum dependent parton distributions within a $3 Q$ light-cone picture of the nucleon, JHEP 05 (2011) 041 [arXiv: 1102.4704] [INSPIRE].

[90] R. Kundu and A. Metz, Higher twist and transverse momentum dependent parton distributions: A Light front Hamiltonian approach, Phys. Rev. D 65 (2002) 014009 [hep-ph/0107073] [INSPIRE].

[91] M. Schlegel and A. Metz, On the validity of Lorentz invariance relations between parton distributions, in Proceedings, 10th Advanced Research Workshop on High-Energy Spin Physics (SPIN-03), Dubna, Russia, September 16-20, 2003, pp. 169-173 (2004) [hep-ph/0406289] [INSPIRE].

[92] K. Goeke, A. Metz, P.V. Pobylitsa and M.V. Polyakov, Lorentz invariance relations among parton distributions revisited, Phys. Lett. B 567 (2003) 27 [hep-ph/0302028] [INSPIRE].

[93] C. Lorcé, B. Pasquini and P. Schweitzer, Unpolarized transverse momentum dependent parton distribution functions beyond leading twist in quark models, JHEP 01 (2015) 103 [arXiv:1411.2550] [INSPIRE].

[94] C. Lorcé, B. Pasquini and P. Schweitzer, Transverse pion structure beyond leading twist in constituent models, Eur. Phys. J. C 76 (2016) 415 [arXiv:1605.00815] [InSPIRE].

[95] P. Zavada, The Structure functions and parton momenta distribution in the hadron rest system, Phys. Rev. D 55 (1997) 4290 [hep-ph/9609372] [inSPIRE].

[96] A.V. Efremov, P. Schweitzer, O.V. Teryaev and P. Zavada, The relation between TMDs and PDFs in the covariant parton model approach, Phys. Rev. D 83 (2011) 054025 [arXiv: 1012.5296] [INSPIRE].

[97] A.V. Efremov, P. Schweitzer, O.V. Teryaev and P. Zavada, Transverse momentum dependent distribution functions in a covariant parton model approach with quark orbital motion, Phys. Rev. D 80 (2009) 014021 [arXiv:0903.3490] [InSPIRE].

[98] P. Schweitzer, Chirally-odd twist-3 distribution function $e^{a}(x)$ in the chiral quark-soliton model, Phys. Rev. D 67 (2003) 114010 [hep-ph/0303011] [INSPIRE].

[99] Y. Ohnishi and M. Wakamatsu, $\pi N$ sigma term and chira-odd twist-3 distribution function $e(x)$ of the nucleon in the chiral quark soliton model, Phys. Rev. D 69 (2004) 114002 [hep-ph/0312044] [INSPIRE].

[100] C. Cebulla, J. Ossmann, P. Schweitzer and D. Urbano, The Twist-3 Parton Distribution Function $e^{a}(x)$ in Large- $N_{c}$ Chiral Theory, Acta Phys. Polon. B 39 (2008) 609 [arXiv:0710.3103] [INSPIRE].

[101] S. Meissner, A. Metz and K. Goeke, Relations between generalized and transverse momentum dependent parton distributions, Phys. Rev. D 76 (2007) 034002 [hep-ph/0703176] [INSPIRE].

[102] A. Mukherjee, Twist-three Distribution e(x): Sum Rules and Equation of Motion Relations, Phys. Lett. B 687 (2010) 180 [arXiv:0912.1446] [INSPIRE].

[103] A. Harindranath and W.-M. Zhang, Examination of Wandzura-Wilczek relation for $g_{2}\left(x, Q^{2}\right)$ in $p Q C D$, Phys. Lett. B 408 (1997) 347 [hep-ph/9706419] [INSPIRE]. 
[104] D. Boer, P.J. Mulders and F. Pijlman, Universality of T-odd effects in single spin and azimuthal asymmetries, Nucl. Phys. B 667 (2003) 201 [hep-ph/0303034] [INSPIRE].

[105] X. Ji, J.-W. Qiu, W. Vogelsang and F. Yuan, Unified Picture for Single Transverse-Spin Asymmetries in Hard-Scattering Processes, Phys. Rev. Lett. 97 (2006) 082002 [hep-ph/0602239] [INSPIRE].

[106] A. Ali, V.M. Braun and G. Hiller, Asymptotic solutions of the evolution equation for the polarized nucleon structure function $g_{2}\left(x, Q^{2}\right)$, Phys. Lett. B 266 (1991) 117 [INSPIRE].

[107] Y. Koike and K. Tanaka, $Q^{2}$ evolution of nucleon's chiral odd twist-three structure function: $h_{L}\left(x, Q^{2}\right)$, Phys. Rev. D 51 (1995) 6125 [hep-ph/9412310] [INSPIRE].

[108] I.I. Balitsky, V.M. Braun, Y. Koike and K. Tanaka, $Q^{2}$ evolution of chiral odd twist-three distributions $h_{L}\left(x, Q^{2}\right)$ and $e\left(x, Q^{2}\right)$ in the large $N_{c}$ limit, Phys. Rev. Lett. 77 (1996) 3078 [hep-ph/9605439] [INSPIRE].

[109] A.V. Belitsky and D. Müller, Scale dependence of the chiral odd twist-three distributions $h_{L}(x)$ and $e(x)$, Nucl. Phys. B 503 (1997) 279 [hep-ph/9702354] [InSPIRE].

[110] M. Anselmino, M. Boglione, U. D'Alesio, A. Kotzinian, F. Murgia and A. Prokudin, The role of Cahn and Sivers effects in deep inelastic scattering, Phys. Rev. D 71 (2005) 074006 [hep-ph/0501196] [INSPIRE].

[111] J.C. Collins, A.V. Efremov, K. Goeke, S. Menzel, A. Metz and P. Schweitzer, Sivers effect in semiinclusive deeply inelastic scattering, Phys. Rev. D 73 (2006) 014021 [hep-ph/0509076] [INSPIRE].

[112] U. D'Alesio and F. Murgia, Azimuthal and single spin asymmetries in hard scattering processes, Prog. Part. Nucl. Phys. 61 (2008) 394 [arXiv:0712.4328] [INSPIRE].

[113] P. Schweitzer, T. Teckentrup and A. Metz, Intrinsic transverse parton momenta in deeply inelastic reactions, Phys. Rev. D 81 (2010) 094019 [arXiv: 1003.2190] [INSPIRE].

[114] A. Signori, A. Bacchetta, M. Radici and G. Schnell, Investigations into the flavor dependence of partonic transverse momentum, JHEP 11 (2013) 194 [arXiv:1309.3507] [INSPIRE].

[115] M. Anselmino, M. Boglione, J.O. Gonzalez Hernandez, S. Melis and A. Prokudin, Unpolarised transverse momentum dependent distribution and fragmentation functions from SIDIS multiplicities, JHEP 04 (2014) 005 [arXiv: 1312.6261] [INSPIRE].

[116] A. Bacchetta, D. Boer, M. Diehl and P.J. Mulders, Matches and mismatches in the descriptions of semi-inclusive processes at low and high transverse momentum, JHEP 08 (2008) 023 [arXiv: 0803.0227] [INSPIRE].

[117] F. Landry, R. Brock, P.M. Nadolsky and C.P. Yuan, Tevatron run-1 Z boson data and Collins-Soper-Sterman resummation formalism, Phys. Rev. D 67 (2003) 073016 [hep-ph/0212159] [INSPIRE].

[118] A.D. Martin, W.J. Stirling, R.S. Thorne and G. Watt, Parton distributions for the LHC, Eur. Phys. J. C 63 (2009) 189 [arXiv:0901.0002] [InSPIRE].

[119] D. de Florian, R. Sassot and M. Stratmann, Global analysis of fragmentation functions for pions and kaons and their uncertainties, Phys. Rev. D 75 (2007) 114010 [hep-ph/0703242] [INSPIRE].

[120] P. Schweitzer, M. Strikman and C. Weiss, Intrinsic transverse momentum and parton correlations from dynamical chiral symmetry breaking, JHEP 01 (2013) 163 [arXiv: 1210.1267] [INSPIRE]. 
[121] J. Collins, L. Gamberg, A. Prokudin, T.C. Rogers, N. Sato and B. Wang, Relating transverse-momentum-dependent and collinear factorization theorems in a generalized formalism, Phys. Rev. D 94 (2016) 034014 [arXiv:1605.00671] [INSPIRE].

[122] CLAS collaboration, Measurement of unpolarized semi-inclusive $\pi^{+}$electroproduction off the proton, Phys. Rev. D 80 (2009) 032004 [arXiv:0809.1153] [INSPIRE].

[123] HERMES collaboration, Multiplicities of charged pions and kaons from semi-inclusive deep-inelastic scattering by the proton and the deuteron, Phys. Rev. D 87 (2013) 074029 [arXiv: 1212.5407] [INSPIRE].

[124] COMPASS collaboration, Transverse-momentum-dependent multiplicities of charged hadrons in muon-deuteron deep inelastic scattering, Phys. Rev. D 97 (2018) 032006 [arXiv: 1709.07374] [INSPIRE].

[125] A. Bacchetta, F. Delcarro, C. Pisano, M. Radici and A. Signori, Extraction of partonic transverse momentum distributions from semi-inclusive deep-inelastic scattering, Drell-Yan and Z-boson production, JHEP 06 (2017) 081 [arXiv:1703.10157] [INSPIRE].

[126] CLAS collaboration, Measurement of Single- and Double-Spin Asymmetries in Deep Inelastic Pion Electroproduction with a Longitudinally Polarized Target, Phys. Rev. Lett. 105 (2010) 262002 [arXiv: 1003.4549] [INSPIRE].

[127] HERMES collaboration, Longitudinal double-spin asymmetries in semi-inclusive deep-inelastic scattering of electrons and positrons by protons and deuterons, arXiv: 1810.07054 [INSPIRE].

[128] COMPASS collaboration, Azimuthal asymmetries of charged hadrons produced in high-energy muon scattering off longitudinally polarised deuterons, Eur. Phys. J. C 78 (2018) 952 [arXiv: 1609.06062] [inSPIRE].

[129] D.W. Sivers, Single-spin production asymmetries from the hard scattering of pointlike constituents, Phys. Rev. D 41 (1990) 83 [InSPIRE].

[130] M. Anselmino, M. Boglione, U. D’Alesio, S. Melis, F. Murgia and A. Prokudin, New insight on the Sivers transverse momentum dependent distribution function, J. Phys. Conf. Ser. 295 (2011) 012062 [arXiv: 1012.3565] [INSPIRE].

[131] M. Anselmino, M. Boglione, U. D'Alesio, A. Kotzinian, F. Murgia and A. Prokudin, Extracting the Sivers function from polarized semi-inclusive deep inelastic scattering data and making predictions, Phys. Rev. D 72 (2005) 094007 [Erratum ibid. D 72 (2005) 099903] [hep-ph/0507181] [INSPIRE].

[132] M. Anselmino et al., Comparing extractions of Sivers functions, in Transversity. Proceedings, Workshop, Como, Italy, September 7-10, 2005, pp. 236-243 (2005) [DOI:10.1142/9789812773272_0028] [hep-ph/0511017] [INSPIRE].

[133] W. Vogelsang and F. Yuan, Single-transverse-spin asymmetries: From deep inelastic scattering to hadronic collisions, Phys. Rev. D 72 (2005) 054028 [hep-ph/0507266] [INSPIRE].

[134] M. Anselmino et al., Sivers effect for pion and kaon production in semi-inclusive deep inelastic scattering, Eur. Phys. J. A 39 (2009) 89 [arXiv:0805.2677] [INSPIRE].

[135] A. Bacchetta and M. Radici, Constraining Quark Angular Momentum through Semi-Inclusive Measurements, Phys. Rev. Lett. 107 (2011) 212001 [arXiv:1107.5755] [INSPIRE]. 
[136] M. Anselmino, M. Boglione, U. D'Alesio, S. Melis, F. Murgia and A. Prokudin, Sivers Distribution Functions and the Latest SIDIS Data, in 19th International Workshop on Deep-Inelastic Scattering and Related Subjects (DIS 2011), Newport News, Virginia, April 11-15, 2011 (2011) [arXiv:1107.4446] [INSPIRE].

[137] HERMES collaboration, Observation of the Naive-T-odd Sivers Effect in Deep-Inelastic Scattering, Phys. Rev. Lett. 103 (2009) 152002 [arXiv:0906.3918] [INSPIRE].

[138] COMPASS collaboration, II - Experimental investigation of transverse spin asymmetries in $\mu$-p SIDIS processes: Sivers asymmetries, Phys. Lett. B 717 (2012) 383 [arXiv:1205.5122] [INSPIRE].

[139] M. Anselmino, M. Boglione and S. Melis, Strategy towards the extraction of the Sivers function with transverse momentum dependent evolution, Phys. Rev. D 86 (2012) 014028 [arXiv: 1204.1239] [INSPIRE].

[140] S.J. Brodsky, D.S. Hwang and I. Schmidt, Final-state interactions and single-spin asymmetries in semi-inclusive deep inelastic scattering, Phys. Lett. B 530 (2002) 99 [hep-ph/0201296] [INSPIRE].

[141] S.J. Brodsky, D.S. Hwang and I. Schmidt, Initial-state interactions and single-spin asymmetries in Drell-Yan processes, Nucl. Phys. B 642 (2002) 344 [hep-ph/0206259] [INSPIRE].

[142] STAR collaboration, Measurement of the transverse single-spin asymmetry in $p^{\uparrow}+p \rightarrow W^{ \pm} / Z^{0}$ at RHIC, Phys. Rev. Lett. 116 (2016) 132301 [arXiv:1511.06003] [INSPIRE].

[143] COMPASS collaboration, First Measurement of Transverse-Spin-Dependent Azimuthal Asymmetries in the Drell-Yan Process, Phys. Rev. Lett. 119 (2017) 112002 [arXiv: 1704.00488] [INSPIRE].

[144] B. Parsamyan, First measurement of transverse-spin-dependent azimuthal asymmetries in the Drell-Yan process, PoS (DIS2017) 243 (2018) [arXiv:1801.01487] [INSPIRE].

[145] A. Bacchetta and M. Radici, Partial-wave analysis of two-hadron fragmentation functions, Phys. Rev. D 67 (2003) 094002 [hep-ph/0212300] [InSPIRE].

[146] A. Bacchetta and M. Radici, Two-hadron semi-inclusive production including subleading twist contributions, Phys. Rev. D 69 (2004) 074026 [hep-ph/0311173] [INSPIRE].

[147] A. Bacchetta, A. Courtoy and M. Radici, First Glances at the Transversity Parton Distribution through Dihadron Fragmentation Functions, Phys. Rev. Lett. 107 (2011) 012001 [arXiv: 1104.3855] [inSPIRE].

[148] A. Bacchetta, A. Courtoy and M. Radici, First extraction of valence transversities in a collinear framework, JHEP 03 (2013) 119 [arXiv:1212.3568] [inSPIRE].

[149] M. Radici, A. Courtoy, A. Bacchetta and M. Guagnelli, Improved extraction of valence transversity distributions from inclusive dihadron production, JHEP 05 (2015) 123 [arXiv: 1503.03495] [INSPIRE].

[150] M. Radici and A. Bacchetta, First Extraction of Transversity from a Global Analysis of Electron-Proton and Proton-Proton Data, Phys. Rev. Lett. 120 (2018) 192001 [arXiv: 1802.05212] [INSPIRE].

[151] J.C. Collins, Fragmentation of transversely polarized quarks probed in transverse momentum distributions, Nucl. Phys. B 396 (1993) 161 [hep-ph/9208213] [INSPIRE]. 
[152] M. Anselmino, M. Boglione, U. D’Alesio, S. Melis, F. Murgia and A. Prokudin, Simultaneous extraction of transversity and Collins functions from new SIDIS and $e^{+} e^{-}$ data, Phys. Rev. D 87 (2013) 094019 [arXiv:1303.3822] [INSPIRE].

[153] Z.-B. Kang, A. Prokudin, P. Sun and F. Yuan, Nucleon tensor charge from Collins azimuthal asymmetry measurements, Phys. Rev. D 91 (2015) 071501 [arXiv:1410.4877] [INSPIRE].

[154] M. Anselmino et al., Collins functions for pions from SIDIS and new $e^{+} e^{-}$data: a first glance at their transverse momentum dependence, Phys. Rev. D 92 (2015) 114023 [arXiv: 1510.05389 ] [INSPIRE].

[155] HERMES collaboration, Effects of transversity in deep-inelastic scattering by polarized protons, Phys. Lett. B 693 (2010) 11 [arXiv:1006.4221] [INSPIRE].

[156] COMPASS collaboration, Collins and Sivers asymmetries in muonproduction of pions and kaons off transversely polarised protons, Phys. Lett. B 744 (2015) 250 [arXiv: 1408.4405] [INSPIRE].

[157] V. Barone, S. Melis and A. Prokudin, The Boer-Mulders effect in unpolarized SIDIS: An Analysis of the COMPASS and HERMES data on the cos $2 \phi$ asymmetry, Phys. Rev. D 81 (2010) 114026 [arXiv:0912 .5194] [INSPIRE].

[158] HERMES collaboration, Azimuthal distributions of charged hadrons, pions, and kaons produced in deep-inelastic scattering off unpolarized protons and deuterons, Phys. Rev. D 87 (2013) 012010 [arXiv: 1204.4161] [INSPIRE].

[159] COMPASS collaboration, Measurement of azimuthal hadron asymmetries in semi-inclusive deep inelastic scattering off unpolarised nucleons, Nucl. Phys. B 886 (2014) 1046 [arXiv:1401.6284] [INSPIRE].

[160] R.N. Cahn, Azimuthal dependence in leptoproduction: A simple parton model calculation, Phys. Lett. 78B (1978) 269 [inSPIRE].

[161] X. Cao, Parton distributions and $\cos 2 \phi_{h}$ asymmetry induced by anomalous photon-quark coupling, Phys. Rev. D 97 (2018) 114010 [arXiv:1802.02417] [INSPIRE].

[162] V. Barone, S. Melis and A. Prokudin, Azimuthal asymmetries in unpolarized Drell-Yan processes and the Boer-Mulders distributions of antiquarks, Phys. Rev. D 82 (2010) 114025 [arXiv: 1009.3423] [INSPIRE].

[163] V. Barone, M. Boglione, J.O. Gonzalez Hernandez and S. Melis, Phenomenological analysis of azimuthal asymmetries in unpolarized semi-inclusive deep inelastic scattering, Phys. Rev. D 91 (2015) 074019 [arXiv: 1502.04214] [INSPIRE].

[164] H. Avakian, A.V. Efremov, P. Schweitzer and F. Yuan, Transverse momentum dependent distribution function $h_{1 T}^{\perp}$ and the single spin asymmetry $A_{U T}^{\sin \left(3 \phi-\phi_{S}\right)}$, Phys. Rev. D 78 (2008) 114024 [arXiv:0805.3355] [INSPIRE].

[165] J. She, J. Zhu and B.-Q. Ma, $h_{1 T}^{\perp}$ and quark orbital angular momentum, Phys. Rev. D 79 (2009) 054008 [arXiv:0902.3718] [INSPIRE].

[166] C. Lorcé and B. Pasquini, The pretzelosity TMD and quark orbital angular momentum, Phys. Lett. B $\mathbf{7 1 0}$ (2012) 486 [arXiv:1111.6069] [InSPIRE].

[167] B. Parsamyan, Transverse spin asymmetries at COMPASS: beyond Collins and Sivers effects, PoS (DIS2013) 231 (2013) [arXiv: 1307.0183] [INSPIRE].

[168] G. Schnell, The Sivers and other semi-inclusive single-spin asymmetries at HERMES, PoS (DIS2010) 247 (2010). 
[169] C. Lefky and A. Prokudin, Extraction of the distribution function $h_{1 T}^{\perp}$ from experimental data, Phys. Rev. D 91 (2015) 034010 [arXiv:1411.0580] [InSPIRE].

[170] A. Kotzinian, SIDIS Asymmetries in Quark-Diquark Model, in Transversity 2008: 2nd International Workshop on Transverse Polarization Phenomena in Hard Processes, Ferrara, Italy, May 28-31, 2008 (2008) [DOI:10.1142/9789814277785_0029] [arXiv:0806.3804] [INSPIRE].

[171] S. Boffi, A.V. Efremov, B. Pasquini and P. Schweitzer, Azimuthal spin asymmetries in light-cone constituent quark models, Phys. Rev. D 79 (2009) 094012 [arXiv:0903.1271] [INSPIRE].

[172] NNPDF collaboration, Parton distributions for the LHC Run II, JHEP 04 (2015) 040 [arXiv:1410.8849] [INSPIRE].

[173] Jefferson Lab Hall A collaboration, Beam-Target Double Spin Asymmetry $A_{L T}$ in Charged Pion Production from Deep Inelastic Scattering on a Transversely Polarized ${ }^{3} \mathrm{He}$ Target at $1.4<Q^{2}<2.7 \mathrm{GeV}^{2}$, Phys. Rev. Lett. 108 (2012) 052001 [arXiv:1108.0489] [INSPIRE].

[174] COMPASS collaboration, New target transverse spin dependent azimuthal asymmetries from COMPASS experiment, Eur. Phys. J. ST 162 (2008) 89 [arXiv:0709.3440] [InSPIRE].

[175] B. Parsamyan, Transverse spin azimuthal asymmetries in SIDIS at COMPASS: Multidimensional analysis, Int. J. Mod. Phys. Conf. Ser. 40 (2016) 1660029 [arXiv: 1504.01599] [INSPIRE].

[176] B. Parsamyan, Measurement of target-polarization dependent azimuthal asymmetries in SIDIS and Drell-Yan processes at COMPASS experiment, PoS(QCDEV2017) 042 (2018).

[177] HERMES collaboration, Measurements of Double-Spin Asymmetries in SIDIS of Longitudinally Polarized Leptons off Transversely Polarized Protons, arXiv:1107.4227 [INSPIRE].

[178] HERMES collaboration, Accessing TMDs at HERMES, AIP Conf. Proc. 1441 (2012) 229 [INSPIRE].

[179] HERMES collaboration, Evidence for a Single-Spin Azimuthal Asymmetry in Semi-inclusive Pion Electroproduction, Phys. Rev. Lett. 84 (2000) 4047 [hep-ex/9910062] [INSPIRE].

[180] B. Parsamyan, Measurement of longitudinal-target-polarization dependent azimuthal asymmetries in SIDIS at COMPASS experiment, PoS (DIS2017) 259 (2018) [arXiv: 1801.01488] [INSPIRE].

[181] CLAS collaboration, Semi-Inclusive $\pi^{0}$ target and beam-target asymmetries from $6 \mathrm{GeV}$ electron scattering with CLAS, Phys. Lett. B 782 (2018) 662 [arXiv:1709.10054] [INSPIRE].

[182] HERMES collaboration, Measurement of single-spin azimuthal asymmetries in semi-inclusive electroproduction of pions and kaons on a longitudinally polarised deuterium target, Phys. Lett. B 562 (2003) 182 [hep-ex/0212039] [INSPIRE].

[183] CLAS collaboration, Measurement of beam-spin asymmetries for $\pi^{+}$electroproduction above the baryon resonance region, Phys. Rev. D 69 (2004) 112004 [hep-ex/0301005] [INSPIRE].

[184] HERMES collaboration, Beam-spin asymmetries in the azimuthal distribution of pion electroproduction, Phys. Lett. B 648 (2007) 164 [hep-ex/0612059] [INSPIRE]. 
[185] W. Gohn, H. Avakian, K. Joo and M. Ungaro, Beam spin asymmetries from semi-inclusive pion electroproduction in deep inelastic scattering, AIP Conf. Proc. 1149 (2009) 461 [INSPIRE].

[186] M. Aghasyan et al., Precise measurements of beam spin asymmetries in semi-inclusive $\pi^{0}$ production, Phys. Lett. B 704 (2011) 397 [arXiv:1106.2293] [INSPIRE].

[187] CLAS collaboration, Beam-spin asymmetries from semi-inclusive pion electroproduction, Phys. Rev. D 89 (2014) 072011 [arXiv:1402.4097] [INSPIRE].

[188] A.V. Efremov, K. Goeke and P. Schweitzer, Azimuthal asymmetries at CLAS: Extraction of $e^{a}(x)$ and prediction of $A_{U L}$, Phys. Rev. D 67 (2003) 114014 [hep-ph/0208124] [INSPIRE].

[189] A. Afanasev and C.E. Carlson, Single spin beam asymmetry in semiexclusive deep inelastic electroproduction, in Intersections of particle and nuclear physics. Proceedings, 8th Conference, CIPANP 2003, New York, U.S.A., May 19-24, 2003 (2003) [hep-ph/0308163] [INSPIRE].

[190] F. Yuan, The Beam single spin asymmetry in semi-inclusive deep inelastic scattering, Phys. Lett. B 589 (2004) 28 [hep-ph/0310279] [INSPIRE].

[191] L.P. Gamberg, D.S. Hwang and K.A. Oganessyan, Chiral-odd fragmentation functions in single pion inclusive electroproduction, Phys. Lett. B 584 (2004) 276 [hep-ph/0311221] [INSPIRE].

[192] A.V. Afanasev and C.E. Carlson, Beam single-spin asymmetry in semiinclusive deep inelastic scattering, Phys. Rev. D 74 (2006) 114027 [hep-ph/0603269] [INSPIRE].

[193] W. Mao and Z. Lu, Beam single spin asymmetry of neutral pion production in semi-inclusive deep inelastic scattering, Phys. Rev. D 87 (2013) 014012 [arXiv:1210.4790] [INSPIRE].

[194] W. Mao and Z. Lu, Beam spin asymmetries of charged and neutral pion production in semi-inclusive DIS, Eur. Phys. J. C 73 (2013) 2557 [arXiv:1306.1004] [INSPIRE].

[195] W. Mao and Z. Lu, On the beam spin asymmetries of electroproduction of charged hadrons off the nucleon targets, Eur. Phys. J. C 74 (2014) 2910 [arXiv:1401.4653] [InSPIRE].

[196] A. Courtoy, Insights into the higher-twist distribution e(x) at CLAS, arXiv:1405.7659 [INSPIRE].

[197] Y. Yang, Z. Lu and I. Schmidt, Twist-3 T-odd fragmentation functions $G^{\perp}$ and $\tilde{G}^{\perp}$ in a spectator model, Phys. Lett. B 761 (2016) 333 [arXiv:1607.01638] [INSPIRE].

[198] B. Pasquini and S. Rodini, The twist-three distribution $e^{q}\left(x, k_{\perp}\right)$ in a light-front model, Phys. Lett. B 788 (2019) 414 [arXiv: 1806.10932] [InSPIRE].

[199] W. Mao, Z. Lu, B.-Q. Ma and I. Schmidt, Double spin asymmetries $A_{L T}^{\cos \phi_{S}}$ and $A_{L T}^{\cos \left(2 \phi_{h}-\phi_{S}\right)}$ in semi-inclusive DIS, Phys. Rev. D 91 (2015) 034029 [arXiv:1412.7390] [INSPIRE].

[200] X. Wang, W. Mao and Z. Lu, Double-spin asymmetry $A_{L T}^{\cos \phi_{S}}$ in semi-inclusive DIS at CLAS12 and EIC within the collinear framework, Phys. Rev. D 94 (2016) 074014 [arXiv: 1606. 01649] [INSPIRE].

[201] M. Anselmino, A. Efremov, A. Kotzinian and B. Parsamyan, Transverse momentum dependence of the quark helicity distributions and the Cahn effect in double-spin asymmetry $A_{L L}$ in semi inclusive DIS, Phys. Rev. D 74 (2006) 074015 [hep-ph/0608048] [INSPIRE]. 
[202] W. Mao, X. Wang, X. Du, Z. Lu and B.-Q. Ma, On the $\cos \phi_{h}$ asymmetry in electroproduction of pions in double longitudinally polarized process, Nucl. Phys. A 945 (2016) 153 [INSPIRE].

[203] HERMES collaboration, Single-spin azimuthal asymmetries in electroproduction of neutral pions in semi-inclusive deep-inelastic scattering, Phys. Rev. D 64 (2001) 097101 [hep-ex/0104005] [INSPIRE].

[204] E. De Sanctis, W.D. Nowak and K.A. Oganesian, Single-spin azimuthal asymmetries in the "Reduced twist-3 approximation", Phys. Lett. B 483 (2000) 69 [hep-ph/0002091] [INSPIRE].

[205] K.A. Oganessian, N. Bianchi, E. De Sanctis and W.D. Nowak, Investigation of single spin asymmetries in $\pi^{+}$electroproduction, Nucl. Phys. A 689 (2001) 784 [hep-ph/0010261] [INSPIRE].

[206] A.V. Efremov, K. Goeke and P. Schweitzer, Predictions for azimuthal asymmetries in pion and kaon production in SIDIS off a longitudinally polarized deuterium target at HERMES, Eur. Phys. J. C 24 (2002) 407 [hep-ph/0112166] [InSPIRE].

[207] A.V. Efremov, K. Goeke and P. Schweitzer, Azimuthal asymmetry in electroproduction of neutral pions in semiinclusive DIS, Phys. Lett. B 522 (2001) 37 [Erratum ibid. B 544 (2002) 389] [hep-ph/0108213] [INSPIRE].

[208] A.V. Efremov, K. Goeke and P. Schweitzer, Azimuthal asymmetries and Collins analyzing power, Nucl. Phys. A 711 (2002) 84 [hep-ph/0206267] [INSPIRE].

[209] B.-Q. Ma, I. Schmidt and J.-J. Yang, Azimuthal spin asymmetries of pion electroproduction, Phys. Rev. D 65 (2002) 034010 [hep-ph/0110324] [INSPIRE].

[210] B.-Q. Ma, I. Schmidt and J.-J. Yang, Reanalysis of azimuthal spin asymmetries of meson electroproduction, Phys. Rev. D 66 (2002) 094001 [hep-ph/0209114] [INSPIRE].

[211] P. Schweitzer and A. Bacchetta, Azimuthal single spin asymmetries in SIDIS in the light of chiral symmetry breaking, Nucl. Phys. A 732 (2004) 106 [hep-ph/0310318] [InSPIRE].

[212] Z. Lu, Single-spin asymmetries in electroproduction of pions on the longitudinally polarized nucleon targets, Phys. Rev. D 90 (2014) 014037 [arXiv:1404.4229] [INSPIRE].

[213] HERMES collaboration, Subleading-twist effects in single-spin asymmetries in semi-inclusive deep-inelastic scattering on a longitudinally polarized hydrogen target, Phys. Lett. B 622 (2005) 14 [hep-ex/0505042] [INSPIRE].

[214] W. Mao, Z. Lu and B.-Q. Ma, Transverse single-spin asymmetries of pion production in semi-inclusive DIS at subleading twist, Phys. Rev. D 90 (2014) 014048 [arXiv:1405.3876] [INSPIRE].

[215] T.P. Cheng and A. Zee, Coincidence Electroproduction and Scaling in the Regge Region, Phys. Rev. D 6 (1972) 885 [INSPIRE].

[216] European Muon collaboration, Measurement of hadronic azimuthal distributions in deep inelastic muon proton scattering, Phys. Lett. 130B (1983) 118 [INSPIRE].

[217] J.T. Dakin et al., Preliminary results on the inclusive electroproduction of hadrons, (1972) [INSPIRE].

[218] H. Mkrtchyan et al., Transverse momentum dependence of semi-inclusive pion production, Phys. Lett. B 665 (2008) 20 [arXiv:0709.3020] [INSPIRE].

[219] M. Anselmino et al., General helicity formalism for polarized semi-inclusive deep inelastic scattering, Phys. Rev. D 83 (2011) 114019 [arXiv:1101.1011] [INSPIRE]. 
[220] A.V. Efremov, K. Goeke, S. Menzel, A. Metz and P. Schweitzer, Sivers effect in semi-inclusive DIS and in the Drell-Yan process, Phys. Lett. B 612 (2005) 233 [hep-ph/0412353] [INSPIRE].

[221] L. Gamberg, Z.-B. Kang and A. Prokudin, Indication on the Process Dependence of the Sivers Effect, Phys. Rev. Lett. 110 (2013) 232301 [arXiv:1302.3218] [INSPIRE].

[222] P. Sun and F. Yuan, Energy evolution for the Sivers asymmetries in hard processes, Phys. Rev. D 88 (2013) 034016 [arXiv: 1304.5037] [INSPIRE].

[223] M. Glück, E. Reya, M. Stratmann and W. Vogelsang, Models for the polarized parton distributions of the nucleon, Phys. Rev. D 63 (2001) 094005 [hep-ph/0011215] [INSPIRE]. 